\title{
Mechanisms of programmed ribosomal -1 frameshifting in bacteria
}

\author{
Dissertation \\ for the award of the degree \\ "Doctor rerum naturalium" (Dr.rer.nat.) \\ Division of Mathematics and Natural Sciences \\ the doctoral program Molecular Biology \\ of the Georg-August-Universität Göttingen
}

Submitted by

Neva Caliskan

from Ankara, Turkey

Göttingen, 2013 
Members of the Thesis Committee:

Prof. Dr. Marina V. Rodnina ( $1^{\text {st }}$ reviewer)

Department of Physical Biochemistry

Max Planck Institute for Biophysical Chemistry

Prof. Dr. Holger Stark ( $2^{\text {nd }}$ reviewer)

3D-Cryo Electron Microscopy

Max Planck Institute for Biophysical Chemistry

Prof. Dr. Ralf Ficner

Department of Molecular Structural Biology

Göttingen Center for Molecular Biosciences

Date of the oral examination: May $29^{\text {th }}, 2013$ 


\section{Affidavit}

I hereby declare that I prepared the dissertation "Mechanisms of programmed ribosomal -1 frameshifting in bacteria" on my own and with no other sources and aids than quoted.

Neva Caliskan

Göttingen, April $15^{\text {th }}, 2013$ 


\section{TABLE OF CONTENTS}

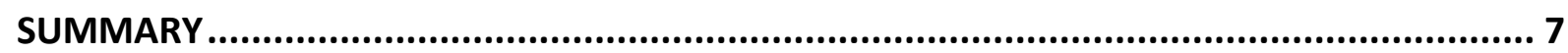

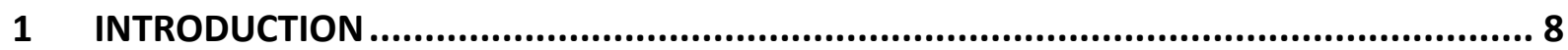

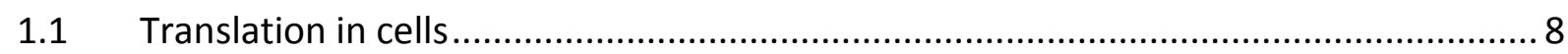

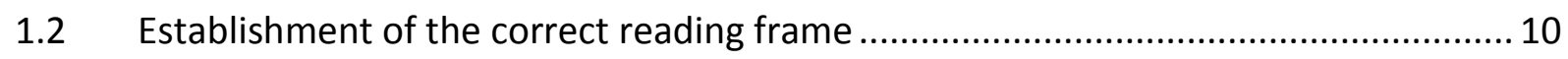

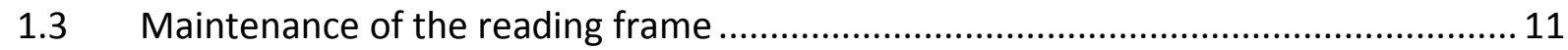

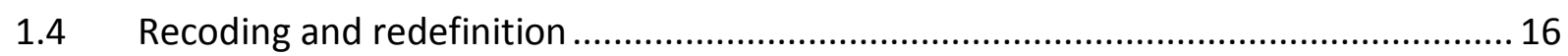

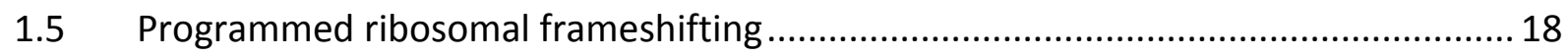

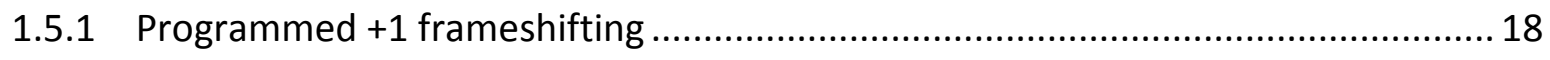

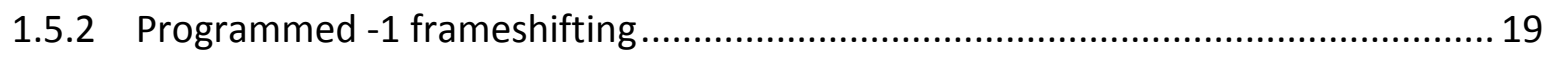

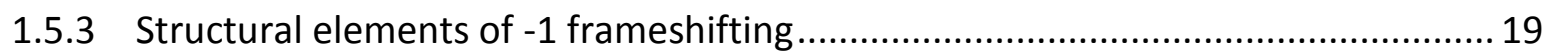

1.6 Models of programmed ribosomal frameshifting..................................................... 22

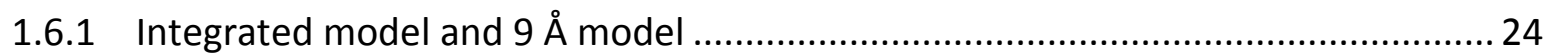

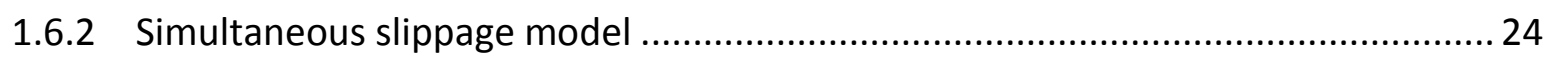

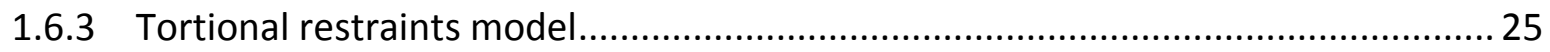

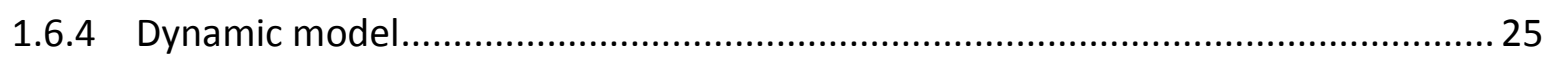

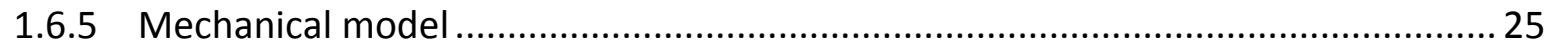

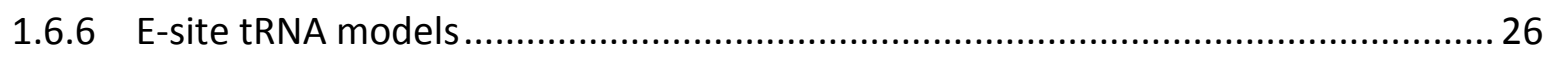

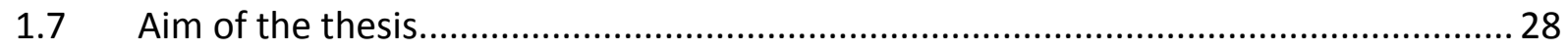

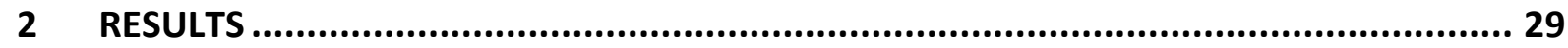

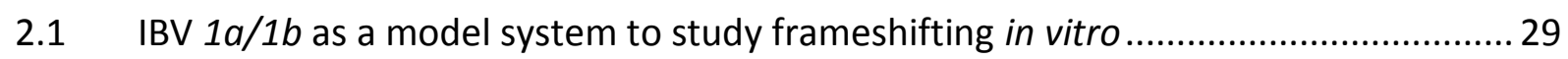

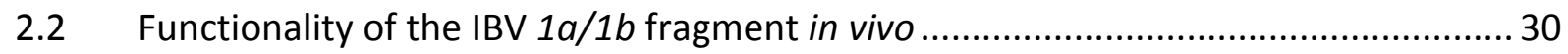

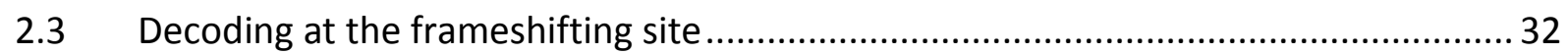

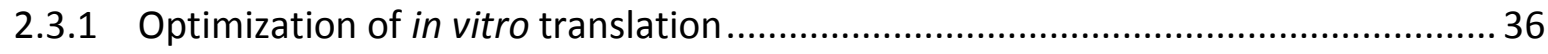

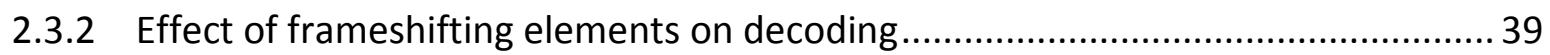

2.3.3 Decoding of lysine at the frameshifting site ...................................................... 43 
2.3.4 Testing the effect of tRNAs decoding -1 and 0 frames following frameshifting 45

2.4 Time-resolved puromycin reactivity and $50 \mathrm{~S}$ translocation at the frameshifting codon 47

2.5 Movement of frameshifting tRNA ${ }^{\text {Lys }}$ from the A to the P site.................................. 51

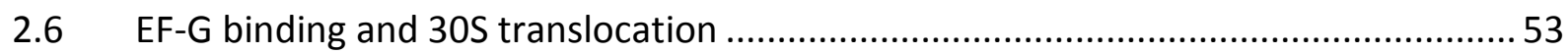

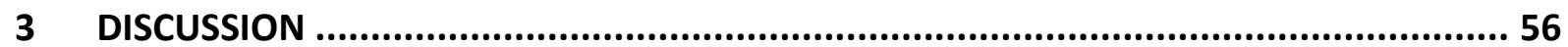

3.1 Functionality of modified IBV $1 a / 1 b$ frameshifting site in E. coli ............................. 56

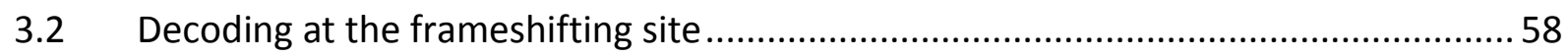

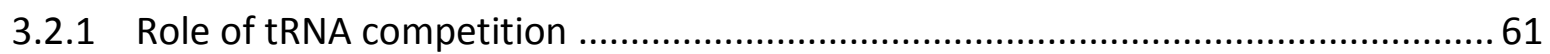

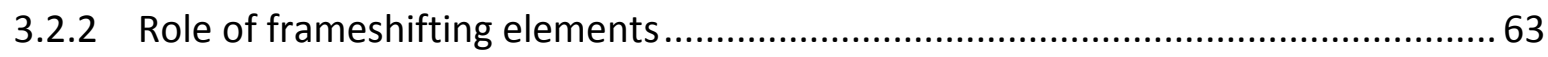

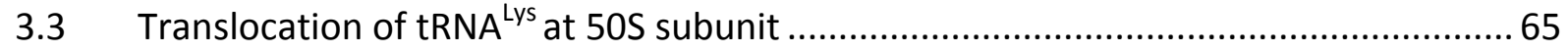

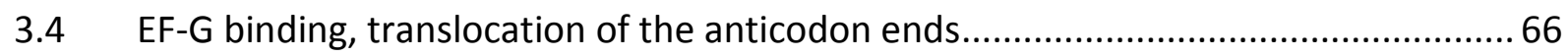

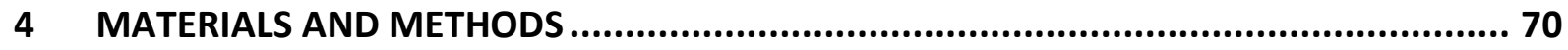

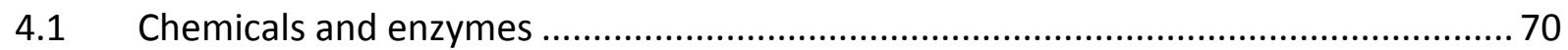

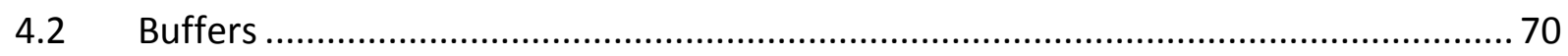

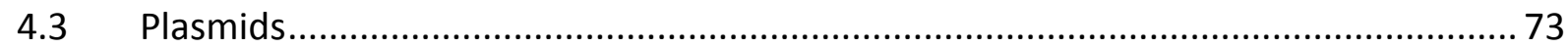

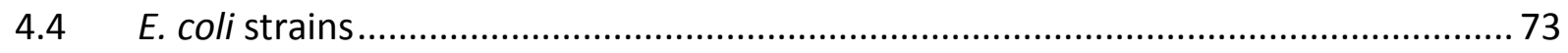

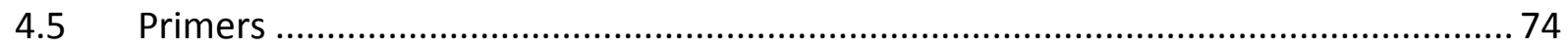

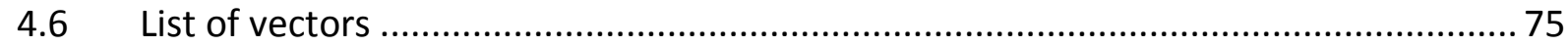

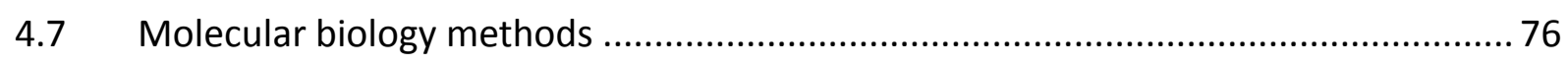

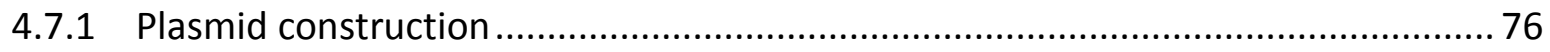

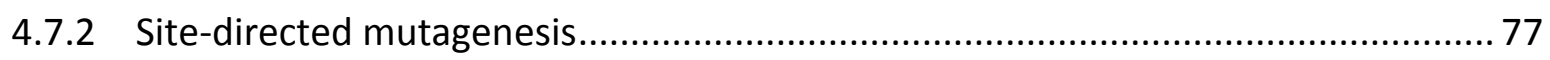

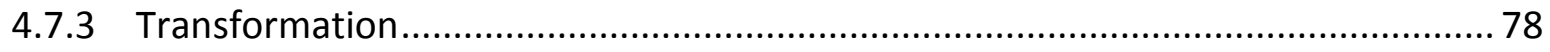

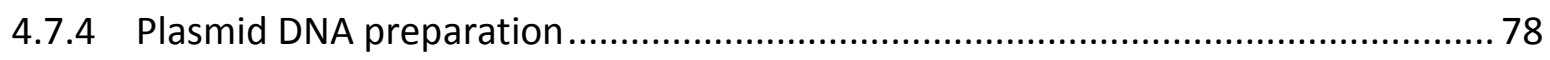

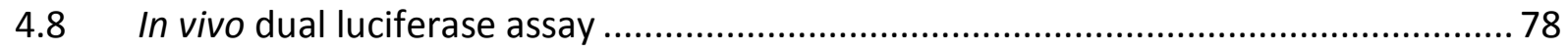

4.9 Preparation of labeled ribosomes (L7/12 138Alx488) and EF-G (WT and 209QSY9) .. 80 


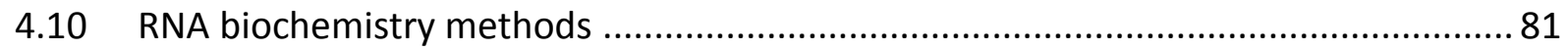

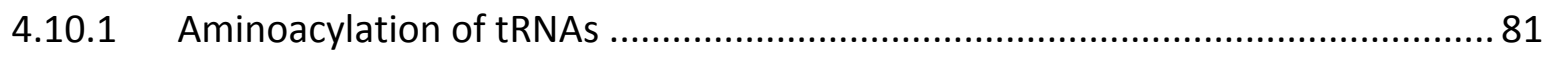

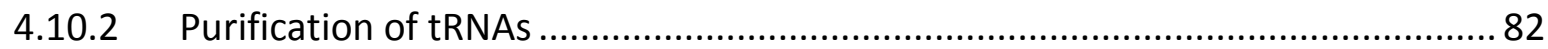

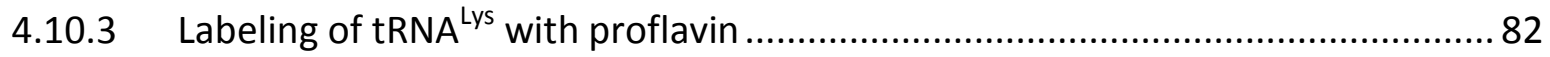

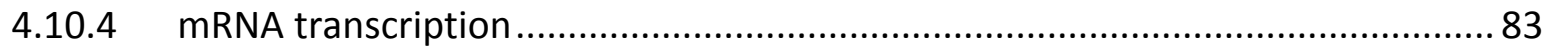

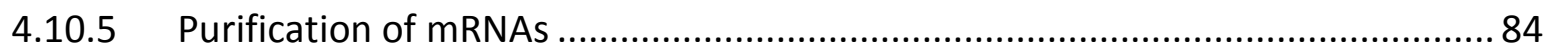

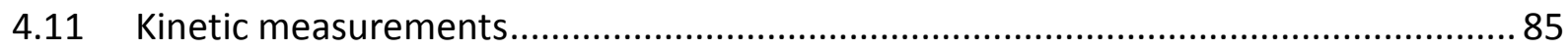

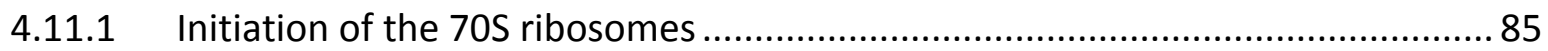

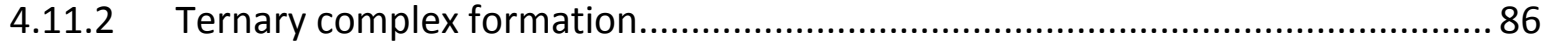

4.11.3 Kinetics of amino acid incorporation measured by quench flow ....................... 86

4.11.4 Separation and quantification of the translation products ................................ 88

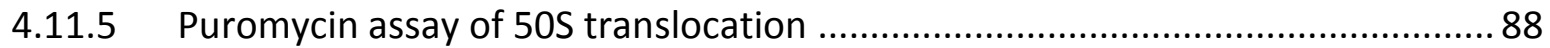

4.11.6 Kinetics of translocation measured by stopped-flow ....................................... 90

4.11.7 Kinetics of EF-G binding measured using stopped flow ................................... 90

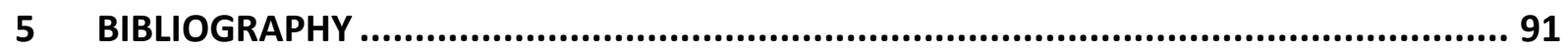

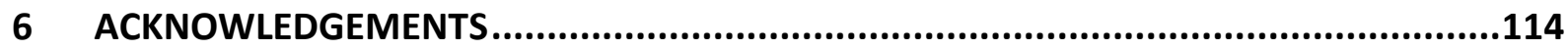

APPENDIX A: IN VIVO DUAL LUCIFERASE ASSAY OF -1 FRAMESHIFTING IN DNAX ............116

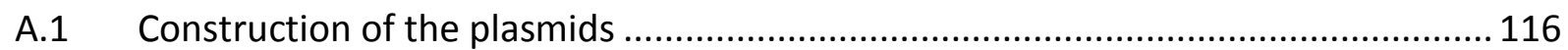

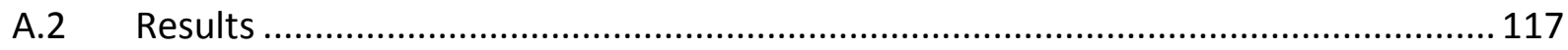

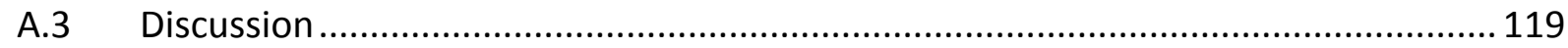

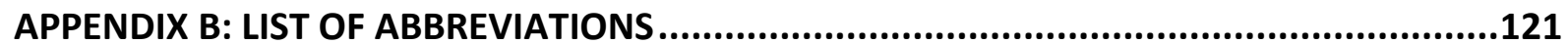

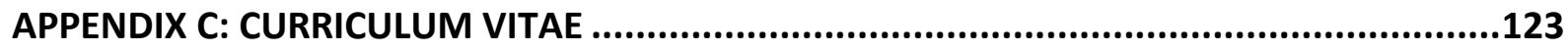




\section{SUMMARY}

Translation of proteins must be accurate to synthesize functional proteins. However, in some cases gene information is reprogrammed which overwrites the normal decoding rules of translation. One of these cases is programmed ribosomal frameshifting, in which the ribosome moves to an alternative, the overlapping reading frame upon encountering specific signals embedded in the mRNAs. A variety of models has been proposed to explain -1 frameshifting mechanistically. However, it is unclear at which step of the elongation cycle -1 frameshifting takes place.

Here we have examined -1 ribosomal frameshifting in real-time by rapid kinetics using a minimal IBV $1 a / 1 b$ frameshifting system which leads to $70 \%$ frameshifting in vivo measured by a dual luciferase assay. We analyzed the formation of the zero- and -1-frame peptides and compared the efficiency of translation of the frameshifting and control mRNAs. We showed that frameshifting takes place following the incorporation of Lys corresponding to the second codon of the slippery sequence, immediately preceding the recoding site. We show that the efficiency of frameshifting does not depend on the competition or availability of tRNAs binding in zero or -1 frames. The presence of the slippery site alone enhances the rapid decoding from the -1 reading frame. The presence of the pseudoknot alone strongly delays decoding of both zero- and -1 frames following the Lys codon. The analysis of $50 \mathrm{~S}$ translocation by a time-resolved puromycin assay and by a fluorescence based tRNA translocation assay suggests that the translocation on the $50 \mathrm{~S}$ subunit is independent of the presence of frameshifting elements, leaving $30 \mathrm{~S}$ translocation as the only step for frameshifting. Using FRET between the ribosome and EF-G we showed that EF-G remains bound longer on the ribosomes during frameshifting. Together, these results suggest the mechanism and exact timing of -1 frameshifting. 


\section{INTRODUCTION}

\subsection{Translation in cells}

Proteins have essential functions in almost every cellular process. Every protein is built of a chain of amino acids, whose identity is defined by the genetic code. Genetic information necessary for the production of proteins in a cell is stored in DNA, which is organized in genes. Synthesis of a protein encoded in a gene entails two phases. In the first phase, the sense strand of the DNA is transcribed by the RNA polymerase into the complementary messenger RNA (mRNA). In the second phase, the mRNA is translated by conversion of the nucleotide triplets known as codons into amino acids that are polymerized into a peptide chain. Accurate processing of genetic information is critical for the functioning of the cell. However, complete accuracy is never achieved during translation with error rates around $10^{-3}$ to $10^{-4}$ (Kramer and Farabaugh, 2007; Parker, 1989; Rodnina and Wintermeyer, 2001a). Rather, translation is optimized towards high speed on the cost of accuracy. The translation errors are usually well tolerated, because they are not deleterious unless located at the catalytic parts of the proteins.

Protein synthesis in the cells is carried out by a large macromolecular complex called the ribosome, which uses messenger RNA as the template and the aminoacylated transfer RNAs (tRNAs) as substrates (Figure 1). Ribosomes are the most abundant ribonucleoprotein complexes composed of ribosomal ribonucleotides ( $\mathrm{RRNA}$ ) and proteins. Their size varies from 2.6 MDa in prokaryotic cells to 4.5 MDa in eukaryotic cells. Ribosomes are composed of two subunits. In prokaryotes the large subunit has a sedimentation coefficient of $50 \mathrm{~S}$ (Svedberg units) and the small one of 30S. The size of prokaryotic ribosome is 70S. The 50S subunit consists of the 23S rRNA, 5S rRNA and 31 proteins. The $50 \mathrm{~S}$ subunit contains the active center where the catalytic step of peptide bond formation takes place, at a site known as peptidyl transferase center. The 30S subunit consists of the 16S rRNA and a total of 21 proteins. The $30 \mathrm{~S}$ subunit contains the decoding center, where the anticodon of the aminoacyl-tRNA is matched by base pair interactions with the codon on the mRNA. The ribosome contains three stable tRNA-binding sites located between the $30 \mathrm{~S}$ and $50 \mathrm{~S}$ subunits. The A site is where the aminoacyl-tRNA is accepted, the $P$ site holds the tRNA 
bound to the growing polypeptide chain after translocation, and the $E$ site is where the deacylated tRNA is directed on the way to exit the ribosome. Understanding of the dynamics and structure-function relationships of the translation machinery have been increased with the high-resolution structures of the ribosome (Bashan and Yonath, 2008; Schmeing and Ramakrishnan, 2009; Steitz, 2008; Yusupov et al., 2001).

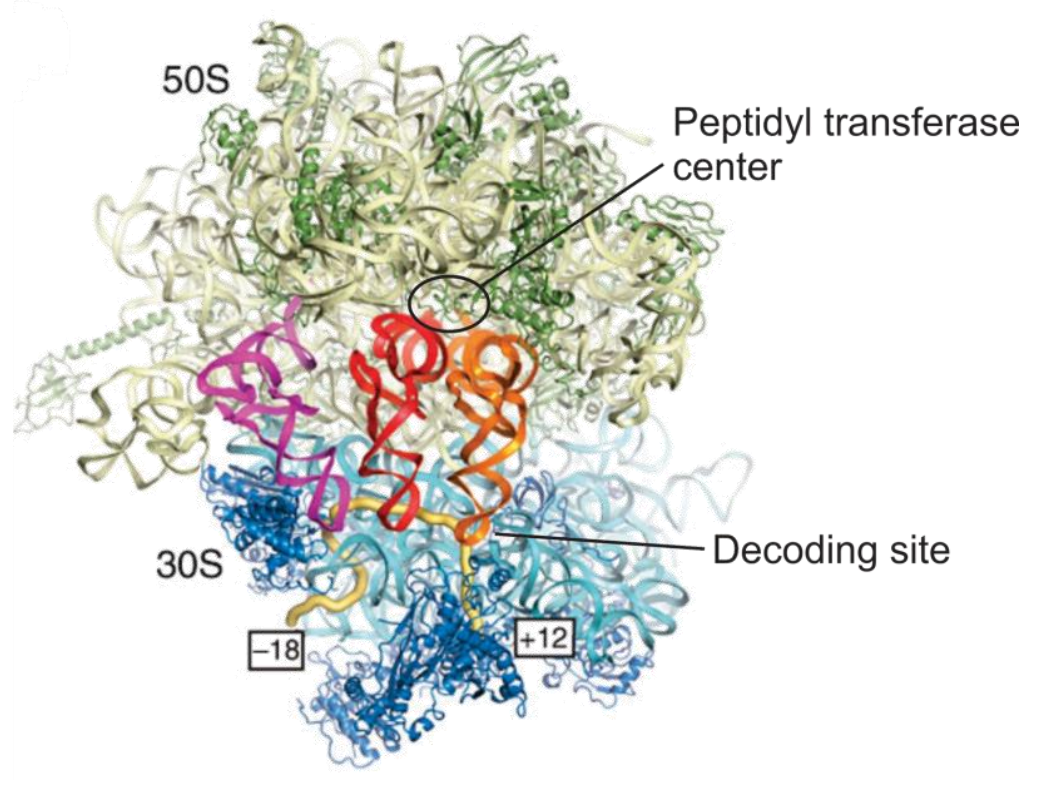

Figure 1. View of the 70S ribosome in the complex with mRNA and tRNAs. $A, P$ and $E$ site tRNAs are shown in orange, red and magenta, respectively. mRNA is depicted in yellow and positions of mRNA entry and exit sites to the ribosome are indicated in boxes (Jenner et al., 2010).

Transfer RNAs (tRNAs) act as adaptor molecules, which associate coding triplets in the mRNA with the individual amino acids. A tRNA is linked to an amino acid by a high energy bond through a process called aminoacylation. In the cell, aminoacylation is performed by aminoacyl-tRNA synthetases, which catalyze the esterification reaction of amino acids with either $2^{\prime} \mathrm{OH}$ or $3^{\prime} \mathrm{OH}$ of the $3^{\prime}$ terminal adenosine residue of the tRNA molecules. Aminoacylated tRNAs can then bind to specific codons on the mRNA, and transfer the activated amino acid to the peptide chain. A universally conserved secondary structure of the tRNA is comprised of the anticodon arm, the $D$ (dihydrouridine) arm, the acceptor arm (where the amino acid binds), the TUC (thymine-pseudouridine-cytosine) arm and the variable arm. Although the general cloverleaf structure is conserved, post-transcriptional 
processing and specific modifications determine the unique features of each tRNA. Several modified nucleosides that are derivatives of $A, U, C, G$ are present in tRNAs from all organisms (Björk, 1995; Juhling et al., 2009). Among those, positions 34 (the wobble base) and 37 ( 3 ' of the anticodon) are especially critical for correct codon-anticodon interactions. Modifications in the tRNAs play important roles in stabilizing the tertiary structure of the tRNA, influencing codon binding, enabling translocation of tRNAs and maintaining the reading frame, thereby affecting the overall speed and accuracy of translation (Agris, 2004, 2008; Byrne et al., 2010; Phelps et al., 2004; Urbonavicius et al., 2001).

A crucial function of the tRNA is to establish correct base-pairing interactions with the mRNA codon. Although the message is translated with high accuracy, the codon-anticodon base pairing between the mRNA and the tRNA does not always follow the Watson-Crick rules (Jones and Nirenberg, 1966). If a perfect Watson-Crick base pair were required at each position in the codon-anticodon complex, we would need 61 tRNA different molecules to read all sense codons in an organism. Instead most organisms have less than 45 tRNA molecules, because a single tRNA can recognize more than one codon due to relaxed recognition rules for the $3^{\text {rd }}$ base pair of each codon, which is called the wobble position (Crick, 1966).

The linear array of ribonucleotides that is specified by a start and a stop codon is referred as the translational reading frame. Given the triplet nature of the mRNA there are three potential reading frames - protein products for each transcript. The translation machinery selects the correct reading frame during translation initiation and engages with it faithfully for each mRNA being translated (Dinman, 2012). Selection of the correct reading frame and maintenance of the reading frame are critical for the functioning of the ribosome.

\subsection{Establishment of the correct reading frame}

Setting of the correct translation initiation site is critical for decoding of the mRNA. In most organisms, the reading frame is assigned by the recognition of the canonical start codon AUG, keeping in mind other codons like UUG and GUG are used occasionally as initiation codons in E. coli (Blattner et al., 1997; Ringquist et al., 1992). In bacteria, translation initiation is facilitated by three initiation factors (IF1, IF2 and IF3) and initiator tRNA (fMet- 
tRNA ${ }^{\text {fMet }}$ ). The ribosome is assisted by the initiation factors to define the mRNA reading frame, and the recruitment of the initiator tRNA, which then establishes the base pairing interactions with the initiation codon (Milon et al., 2012; Rodnina, 2012). In the first phase of initiation, mRNA, IFs and fMet-tRNA ${ }^{f M e t}$ are recruited to the $30 \mathrm{~S}$ subunit, forming a preinitiation complex (pre-IC). Selection of the mRNA partly depends on the structure of the mRNA at the transcription initiation region and the presence of a Shine-Dalgarno (SD) sequence usually 8-10 nucleotides upstream of the initiation codon (Julian et al., 2011; Osterman et al., 2013; Shine and Dalgarno, 1974). Interactions of the $3^{\prime}$ end of the 16S rRNA with the Shine-Dalgarno sequence usually ensures the correct positioning of the start codon at the ribosomal P site (Gold, 1988; Ringquist et al., 1992; Steitz and Jakes, 1975). In the next phase, recognition of the initiation codon by $f M e t-t R N A^{f M e t}$ at the $P$ site stabilizes the conformation of the mRNA within the $30 \mathrm{~S}$ subunit, thereby forming the functional $30 \mathrm{~S}$ initiation complex (IC) (Milon et al., 2012; Simonetti et al., 2009). After the initiator fMettRNA $^{\text {fMet }}$ is positioned at the $\mathrm{P}$ site of the ribosome, the SD helix rearranges its position (Yusupova et al., 2006). These interactions ensure that the initiation codon is read precisely by the translation machinery in the correct reading frame. Lastly, $50 \mathrm{~S}$ subunit is recruited to the $30 \mathrm{~S}$ IC forming the 70S IC, which is ready for translation of the selected mRNA (Grigoriadou et al., 2007; Milon et al., 2008) (Figure 2).

Initiation factors determine the accuracy of initiation at the correct start site. IF2 recruits fMet-tRNA $^{\text {fMet }}$ to the AUG start codon, and hence can be considered as an important player in the reading frame establishment (Milon et al., 2010). IF2 binds to the single-stranded acceptor end of fMet-tRNA ${ }^{\text {fMet; }}$; this single-stranded region acts as a discriminator against elongator tRNAs (Gualerzi et al., 2001). IF2 has a preference for initiator tRNA over the elongator tRNA therefore binding of the initiator tRNA on the 305 subunit is stabilized (Gualerzi and Pon, 1990; Hartz et al., 1990; Hartz et al., 1989). Association of the 50S subunit and dissociation of IF3 are slower on non-canonical initiation codons, which further increases the selectivity for the start codon AUG (Milon et al., 2008).

\subsection{Maintenance of the reading frame}

A critical task during the elongation phase is to maintain the reading frame determined during the initiation phase through the rest of the translation for hundreds to thousands of 
codons. The ribosome has to faithfully read the message in the mRNA in order to produce non-erroneous proteins, which otherwise might have detrimental consequences; such as production of toxic or misfolded proteins. The kinetics of decoding is well characterized by biochemical and biophysical methods (Gromadski and Rodnina, 2004; Gromadski et al., 2002; Pape et al., 1998; Rodnina et al., 2005).

The elongation phase starts with binding of aminoacyl-tRNA to the vacant A site of the ribosome (Figure 2). Aminoacyl-tRNA is delivered to the decoding center of the ribosome in a ternary complex with a translational GTPase called EF-Tu in bacteria and GTP. The aminoacyl-tRNA is delivered to the A site in several steps (Rodnina, 2012; Rodnina and Wintermeyer, 2001a). Initially, ternary complex is recruited through interactions with the C-terminal domain of the L7/L12 stalk (Diaconu et al., 2005; Kothe et al., 2004). After this mRNA codon and tRNA anticodon base pair in the 30S A site. Cognate anticodon-codon base pairing facilitates conformational changes that trigger and enhance the rate of GTP hydrolysis. This is followed by the release of EF-Tu.GDP from the ribosome (Daviter et al., 2006). Release of EF-Tu allows the aminoacyl-tRNA to bind to the 50S A site, which is termed accommodation (Rodnina et al., 1994).

Despite the similarities between decoding properties of tRNAs, not all codons are translated at the same rates. For example, rate of GTP hydrolysis is higher on a cognate Watson-Crick base pair compared to a wobble interaction on the third codon position and the rates differ even more on near-cognate codons (Gromadski et al., 2006). In addition, abundance of a particular aminoacyl-tRNA influences the effective rate of decoding. Altogether, these differences determine the rate of translation of the individual codon and of the protein synthesis as a whole and the miscoding error frequency on individual codons.

Accommodation is followed by almost instantaneous peptide bond formation yielding an $A$ site peptidyl-tRNA one amino acid longer and deacylated-tRNA in the P site (Pape et al., 1998) (Figure 2). Peptide bond formation takes place in the active site (composed of RNA) the peptidyl transferase center - which is located at the 50S subunit of ribosomes (Noller et al., 1992). The reaction is a hydrolysis of an ester bond via nucleophilic attack of the a-amino group of A-site aminoacyl-tRNA on the carbonyl carbon of the ester bond of the P-site peptidyl-tRNA. The ribosome greatly accelerates the catalysis (by 6-7 orders of magnitude), 
mostly by positioning the substrates, electrostatic effects and solvent reorganization (Sharma et al., 2005; Sievers et al., 2004; Trobro and Aqvist, 2005).

The decoding step is critical to ensure the production of correct peptides. However, in some cases non-cognate aminoacyl-tRNA might be accommodated and peptide bond formation would take place with the incorrect amino acid. In this case, there is an additional correction mechanism called retrospective editing. The ribosome is able to sense the codon-anticodon mismatch in the $\mathrm{P}$ site and to stop the synthesis of the erroneous peptide by abortive termination that is stimulated by the activity of release factors (Zaher and Green, 2009).

The following step of translation is the translocation of tRNAs and mRNA through the ribosome which is facilitated by another GTPase called the EF-G (Figure 2). The pretranslocation state ribosome (the PRE state) contains deacylated tRNA in the P site and peptidyl-tRNA in the A site. The complex is highly dynamic and fluctuates between the classical and hybrid states in which the $3^{\prime}$ end of the deacylated tRNA moves from the P site to the $E$ site and of the peptidyl-tRNA from the A site to the $P$ site on the 50 S subunit (Blanchard et al., 2004; Fischer et al., 2010; Julian et al., 2008; Moazed and Noller, 1989; Munro et al., 2007). Large conformational changes of 305 and 505 subunit mediate movement of mRNA and tRNA during translocation (Agrawal et al., 1999). In addition to the inter-subunit rotations, there is also intra-subunit movements of the $30 \mathrm{~S}$ head domain that facilitate tRNA translocation (Ratje et al., 2010).

Binding of EF-G to the ribosome is facilitated by the interactions with the C-terminal domains (CTD) of L7/L12 proteins of the 50S subunit (Savelsbergh et al., 2005). 50S and 30S translocation takes place simultaneously on the ribosome and the formation of the hybrid states is not a prerequisite for EF-G binding (Holtkamp et al. unpublished results). Rotated conformation of the ribosome is stabilized by binding of EF-G (Munro et al., 2010; Valle et al., 2003). Fast GTP hydrolysis by EF-G (Rodnina et al., 1997) precedes and drives translocation of tRNAs by unlocking of the 30S subunit (Savelsbergh et al., 2003). Binding of some antibiotics at the decoding site specifically interfere with the tRNA movement, whereas have no effect on the release of inorganic phosphate $(\mathrm{Pi})$ release indicates that the tRNA-mRNA movement takes place after unlocking (Peske et al., 2004). Back swiveling of the head domain brings the ribosome to the non-rotated conformation, which is termed the 
POST state (Guo and Noller, 2012; Ratje et al., 2010; Savelsbergh et al., 2003; Savelsbergh et al., 2005). The reaction on the $30 \mathrm{~S}$ subunit is completed, most likely by the backwards rotation of the $30 \mathrm{~S}$ head domain and dissociation of EF-G, which requires another conformational change (Cunha, 2012; Savelsbergh et al., 2009). The swiveling movement of the $30 \mathrm{~S}$ subunit head relative to the body is believed to facilitate resolving mRNA secondary structures, because the ribosomal helicase proteins (S3, S4 and S5) are located at the junction of the head and body of the 30S subunit (Takyar et al., 2005; Yusupova et al., 2001). In the presence of EF-G, the directionality of tRNA movement is favored towards the forward movement. Domain IV of EF-G which mimics the anticodon arm of A-site tRNA and binds to the ribosome in a similar fashion as the A-site tRNA (Agrawal et al., 1998; Agrawal et al., 2000; Stark et al., 2000). EF-G interacts with the P-site tRNA and the codon in the posttranslocation state. It was implied that domain 4 of EF-G prevents the back movement of peptidyl-tRNA throughout translocation into the $\mathrm{P}$ site, thereby helps to maintain the reading frame (Gao et al., 2009). Translocation leaves the ribosome with the tRNAs occupying the $E$ site and $P$ site, while the $A$ site is vacant to accept the next aminoacyl-tRNA. When the A site is empty, the mRNA codon in the P site interacts with the bases of the $16 \mathrm{~S}$ rRNA, which ensures that the $P$ site tRNA does not slip and stabilizes the reading frame (Selmer et al., 2006).

The cycle of decoding and translocation takes place repeatedly until a stop codon (UAG, UAA or UGA in bacteria) in the mRNA is recognized by release factors 1 or 2 (RF1 or RF2) (Youngman et al., 2008). RF1 recognizes UAG and UAA stop codons and RF2 recognizes UGA and UAA codons at the A site (Scolnick et al., 1968). The peptide motifs PXT and SPF of RF1 and RF2 are involved in stop codon recognition (Ito et al., 2000). Release factor binding induces a conformation at the peptidyl transferase center, which renders the ester bond of the P-site tRNA accessible to water. The GGQ motif of RFs is critical to assist the water molecule in catalysis. The chemical reaction involves a nucleophilic attack of the water molecule to the carbonyl ester found at the C-terminus of the peptidyl-tRNA, which results in the release of the peptide chain and termination of protein synthesis (Brunelle et al., 2008; Kuhlenkoetter et al., 2011). Dissociation of the release factors from the ribosome is stimulated by RF3 (Grentzmann et al., 1998b) (Figure 2). 
After the release of the nascent peptide chain from the ribosome, ribosome recycling, the last phase of translation cycle takes place. The reaction is facilitated by the ribosome recycling factor (RRF) and EF-G, which accelerate the dissociation of the ribosome into subunits. RRF and EF-G are recruited to the post termination ribosomes containing deacylated tRNA in E/P state (Peske et al., 2005). Rapid GTP hydrolysis by EF-G is followed by a slow release of $\mathrm{Pi}$, which in turn may be related to structural changes for subunit splitting (Savelsbergh et al., 2009) (Figure 2).

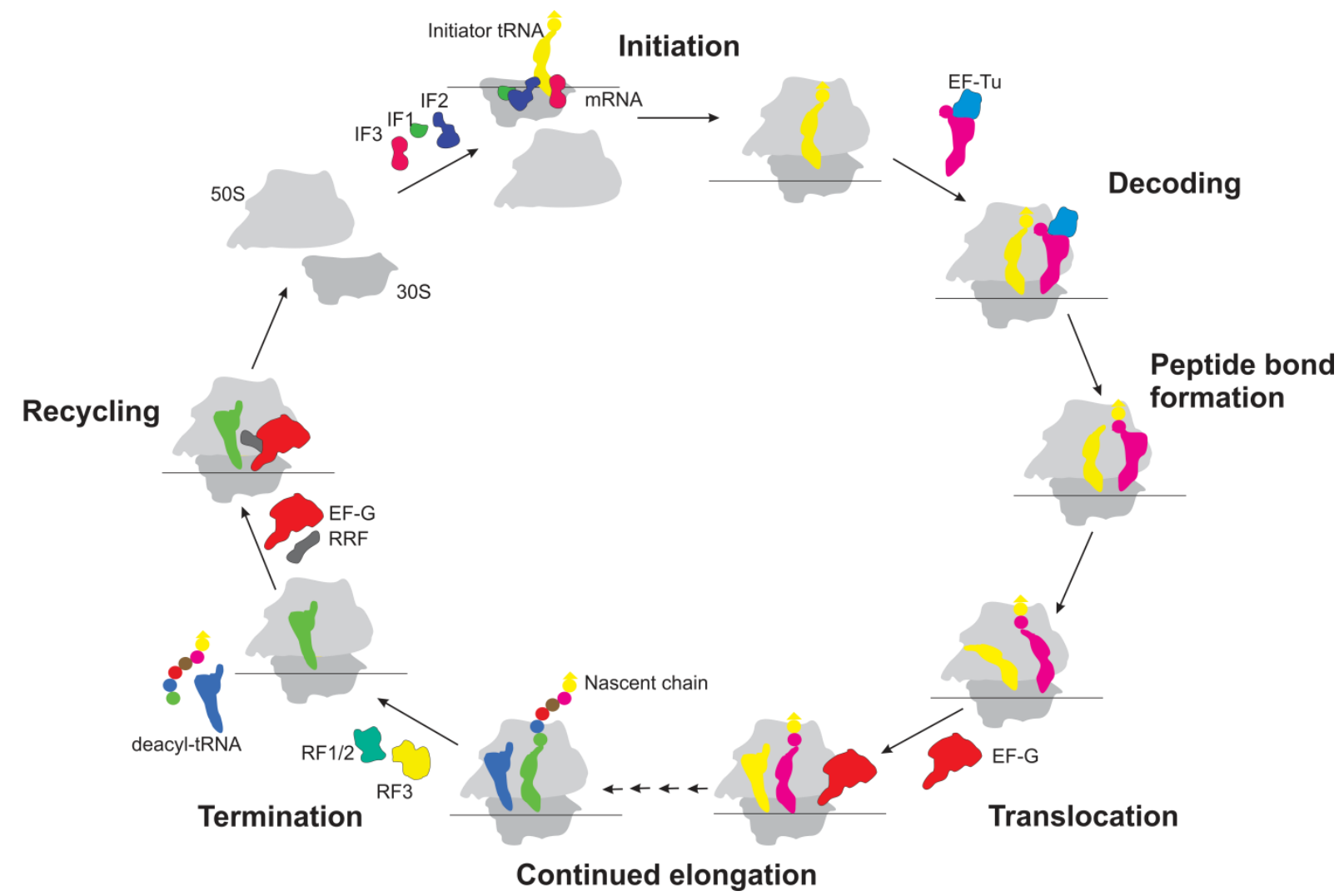

Figure 2. Overview of bacterial translation. For simplicity, only the major steps are shown. The symbols of the individual components of translation (EF-G, EF-Tu, tRNAs, ribosomal subunits etc.) are depicted the same in the following figures.

Biochemical and structural studies suggested many details on the function of ribosomes at different steps of translocation and how fidelity and accuracy is achieved. There are also cases that, at specific signals on certain mRNAs, the translation accuracy is altered in a programmed way. The next section will focus on the mechanisms of programmed recoding and redefinition events. 


\subsection{Recoding and redefinition}

The message embedded in the mRNA is more than the linear array of nucleotides. For instance there are signals which facilitate internal ribosome entry, that bind to repressors, etc. By the signals programmed in mRNA sequences readout of genetic information can be altered by various means. In these cases - defined as recoding - standard rules of decoding are specifically changed upon encountering a signal embedded in the mRNA (Atkins et al., 2001; Atkins and Gesteland, 2009; Baranov et al., 2002a). Recoding can be especially useful to increase diversity of gene expression, because it allows translation of multiple proteins from a single mRNA. This way of manipulating the readout is employed mostly by viruses as they have a rather limited genome size. Moreover, it can be used as a means to regulate gene expression at the post-transcriptional level. Recoding is a programmed mechanism; only a certain proportion of ribosomes use it at a time. The frequency of recoding is kept lower than standard decoding, yet higher than non-programmed recoding or misreading events. Another feature of the recoding signals is the presence of cis-acting secondary structure elements on the mRNAs that stimulate the ribosomes to alter the way an mRNA is decoded (Gesteland and Atkins, 1996).

Recoding strategies can be subdivided in three classes. The first class includes the cases of codon readthrough, which causes changing the meaning of a stop codon. In such cases, upon encountering a stop codon, the ribosome does not terminate translation but continues through the stop codon by inserting an amino acid such as tryptophan, glutamine or selenocysteine using near-cognate or cognate tRNAs. Readthrough was reported mostly in viruses and phages (Feng et al., 1992; Li and Rice, 1993; Weiner and Weber, 1971; Wills et al., 1991). In decoding of developmentally regulated genes of Drosophila melanogaster, readthrough of UGA or UUA stop codons is utilized for production of kel, syn and hdc proteins (Klagges et al., 1996; Robinson and Cooley, 1997; Steneberg and Samakovlis, 2001). Normally, miscoding of stop codons by near-cognate aminoacyl-tRNAs is very inefficient; however, in the case of programmed readthrough the misreading frequency is influenced locally by cis-acting elements. It is interesting that also the first distal nucleotide (especially if it is a C) adjacent to the stop codon influences readthrough efficiency, suggesting an extended stop codon signal responsible for near-cognate aa-tRNA decoding (Bonetti et al., 1995; Li and Rice, 1993). Stop codon readthrough is proposed to be stimulated by modifying 
the decoding efficiency of the suppressor tRNA or by preventing access of release factors to the stop codon. How the secondary structure elements induce the readthrough is not clear. It is possible that entering of eRF1 in the A site is prevented (Namy and Rousset, 2010). Decoding of selenocysteine is another example for the redefinition of stop codons (Bock et al., 1991). In this case, the cognate aa-tRNA for UGA codon specifies decoding of selenocysteine (Tujebajeva et al., 2000). In bacteria, a specific elongation factor SelB recognizes the selenocystine moiety of the tRNA ${ }^{\mathrm{Sec}}$ and forms exclusively the ternary complex with selenocysteyl-tRNA ${ }^{\mathrm{Sec}}$. Presence of the SECIS element, which is a stem loop immediately downstream of the UGA codon facilitates incorporation of Sec. High affinity of SelB to the SECIS element - without additional factors - targets the selenocysteyl-tRNA ${ }^{\text {Sec }}$ to the specific UGA codon (Commans and Böck, 1999; Paleskava et al., 2010).

The second class of recoding is bypassing or hopping which can lead to translation of fusion proteins with two distal open reading frames. This involves dissociation of codon-anticodon interactions at the P site followed by skipping of a portion of the mRNA and reestablishment of interactions at a matching codon downstream. The best studied (and only known) case of bypassing is the bacteriophage T4 gene 60 that encodes a topoisomerase subunit. The coding sequence of the mRNA is interrupted by a gap of 50 nucleotides, which is bypassed by the ribosome to continue translation (Herr et al., 2000; Huang et al., 1988). Bypassing is stimulated by an UAG codon immediately $3^{\prime}$ of the take-off site, a hairpin loop, and a nascent peptide synthesized upstream which facilitates initial dissociation of the codon anticodon base pair, matching take off and landing GGA codons and a Shine-Dalgarno sequence (Wills, 2010). In addition, mutations in ribosomal protein $L 9$ can reverse the effect from the loss of the hairpin loop, thereby enhances bypassing (Farabaugh, 1996a; Herr et al., 2001a). Although most of the ribosomes are reported to take-off during bypassing, not all of them land correctly on the GGA codon (Herr et al., 2001b). The take off is proposed to be initiated through weak $P$ site codon-anticodon interaction at a GGA codon. The scanning phase is followed by landing where a match between P site codon and downstream mRNA can be reestablished (Herr et al., 2000). 


\subsection{Programmed ribosomal frameshifting}

The third class of recoding are programmed frameshifting events, which occur at particular sites in mRNAs with overlapping open reading frames. Unlike missense errors, which might not affect the stability or activity of a protein, non-programmed frameshifting errors are deleterious and kept at low frequency. On the other hand, programmed frameshifting can increase tRNA slippage enormously. In response to specific signals embedded in the mRNA, ribosomes move one base in $3^{\prime}$ or $5^{\prime}$ direction and switch to the alternative reading frame. The stimulatory signals differ from one mRNA to another. However, the general sequence features are conserved and include a slippery site where the shift of the reading frame takes place and a stimulatory secondary structure, usually in the form of a hairpin or a pseudoknot.

\subsubsection{Programmed +1 frameshifting}

Programmed +1 frameshifting has been found in many organisms from bacteria to eukaryotes. In eukaryotes, yeast Ty retrotransposable elements (Belcourt and Farabaugh, 1990; Wilson et al., 1986) and cellular genes such as EST3, OAZ1 and ABP140 utilize +1 frameshifting (Asakura et al., 1998; Matsufuji et al., 1995; Morris and Lundblad, 1997). In bacteria, the frameshift in prf2 mRNA (encoding for RF2) occurs at a slippery sequence CUU UGA by an efficiency of $30-50 \%$ and in this case +1 frameshifting has an autoregulatory function (Baranov et al., 2002b). When the concentration of RF2 in the cell drops, termination is bypassed by +1 frameshifting, resulting in synthesis of full-size RF2, whereas when the RF2 concentration is high, synthesis of RF2 is efficiently terminated (Craigen and Caskey, 1986). Mechanistically, poor termination efficiency on the UGA codon, weak codonanticodon interactions at the P site, availability of cognate aa-tRNA at the A site, secondary structures downstream and in bacteria the presence of an SD-like sequence upstream of the slippery site are the major determinants of +1 frameshifting (Adamski et al., 1993; Curran, 1993; Devaraj and Fredrick, 2010; Farabaugh, 2010; Gurvich et al., 2005; Stahl et al., 2001). The role of secondary structures in stimulating +1 frameshifting is not clear, although they might interfere with the helicase activity of the ribosome (Farabaugh, 2010; Takyar et al., 2005). 


\subsubsection{Programmed -1 frameshifting}

Programmed -1 frameshifting sites were described in viruses including retroviruses (Jacks et al., 1988b), coronaviruses (Brierley et al., 1989), plant viruses (Brault and Miller, 1992) and arteriviruses (Meulenberg et al., 1993). In eukaryotes, frameshifting in a Drosophila retrotransposon element (Danilevskaya et al., 1994), in the mammalian embryonal carcinoma differentiation regulated gene ( $E d r$, human PEG10) (Clark et al., 2007; Manktelow et al., 2005) and in the human paraneoplastic Ma3 gene (Wills et al., 2006) were reported. In bacteria two cellular genes dnaX (Blinkowa and Walker, 1990) and cdd (Mejlhede et al., 1999) are decoded by -1 frameshifting (Farabaugh, 1996a; Farabaugh, 1996b). Computational screening of the frameshifting elements yielded a larger number of genes potentially decoded by frameshifting in various organisms (Hammell et al., 1999; Jacobs et al., 2007; Moon et al., 2004; Theis et al., 2008).

In most viruses frameshifting is involved in production of replicases and insertion elements. In retroviruses, -1 frameshifting is employed for production of a gag-pol fusion protein from a monocystronic mRNA containing two overlapping open reading frames. Gal ORF produces the capsid protein and Pol ORF is responsible for the synthesis of the polymerase and integrase. In other viruses such as coronaviruses the organization is quite different, as the frameshifting product is the replicase and the structural proteins are produced by subgenomic mRNA (Plant et al., 2010; Thiel et al., 2003). Viral frameshifting efficiencies must be adjusted precisely to have proper viral particle assembly and replication. This is a potential target for antiviral therapy, for example interfering with the frameshifting efficiency of HIV has been shown to impair virus replication (Biswas et al., 2004; Dulude et al., 2008; Irvine et al., 1998; Shehu-Xhilaga et al., 2001).

\subsubsection{Structural elements of -1 frameshifting}

Efficient frameshifting relies on sequence elements embedded in the mRNA. One of those elements is the slippery site comprised of a short stretch of nucleotides, usually a heptamer in the form of two homopolymeric triplets with X-XXY-YYZ motif. In vitro mutational studies reported that $X$ can be any nucleotide except $G, Y$ is either $U$ or $A$ and $Z$ is usually a $G$ or $C$ (Brierley et al., 1992; Jacks et al., 1988b). Some studies also suggest that even a larger region 
can be involved in frameshifting, i.e. the codon upstream of the heptanucleotide stretch might influence frameshifting (Bekaert and Rousset, 2005; Leger et al., 2007). The slippery tRNA is proposed to be intrinsically shifty because it allows cognate or near-cognate base pairing at the new reading frame. For instance in prokaryotes $\operatorname{TRNA}^{\text {Lys }}$, which reads AAA and AAG codons, is the preferred tRNA decoded at the second codon of the frameshifting site. $50 \%$ of bacterial insertion sequences contain X-XXA-AAG as the slippery site (Fayet and Prère, 2010). A-AAA-AAG is also the naturally occurring frameshifting slippery site in dnaX gene in E. coli (Tsuchihashi and Brown, 1992). Slippery sequences alone can direct frameshifting, albeit with lower efficiencies, indicating that the propensity to shift is the property of the slippery sequences. However, the efficiency of -1 frameshifting is increased by a second element, the downstream secondary structure. The stimulatory mRNA structure is found approximately 6-9 nucleotides downstream of the slippery site. A diverse array of stimulatory secondary structures is found in different systems. Stem loops are present at the frameshift sites such as in the dnaX gene in E. coli (Tsuchihashi and Brown, 1992), the human astrovirus (Hast-1) (Marczinke et al., 1994), the SIV and the HIV (Marcheschi et al., 2007). Antisense RNA or LNA oligonucleotides can also induce frameshifting in vitro with efficiencies up to $40 \%$ (Howard et al., 2004; Yu et al., 2010). The most common secondary structure observed at the frameshifting sites is an H-type pseudoknot. The H-type pseudoknot topology is formed when the single-stranded loop region of a hairpin basepairs with downstream complementary nucleotides, resulting in two stems S1 and S2 and two loops L1 and L2 (Giedroc and Cornish, 2009). RS virus frameshifting site, which was the first example of -1 frameshifting, contains one of the most complicated structures with an additional loop 3 element at the helical junction. The role of pseudoknots in frameshifting was first reported in the infectious bronchitis virus (IBV) $1 a / 1 b$ gene (Brierley et al., 1989). A remarkable example for a pseudoknot structure was found in barley yellow dwarf virus (BYDV), which forms the stem 2 with basepairing almost 4000 bases downstream (Brierley et al., 2010) (Figure 3F). The details of structures, requirements and the effects of different stimulatory elements show variations among different translational systems (Garcia et al., 1993). For instance, generally the efficiency of frameshifting in eukaryotes is more dependent on the pseudoknot, whereas bacterial frameshift regions, such as IS elements, do not have an absolute requirement for a downstream secondary structure (Fayet and Prère, 2010). 
A

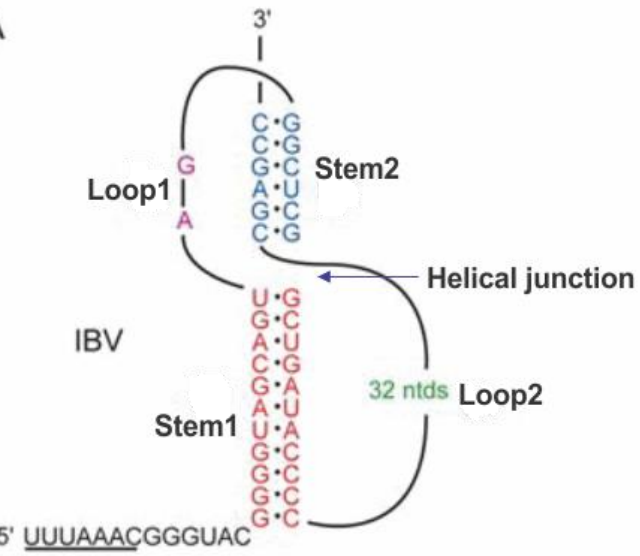

B

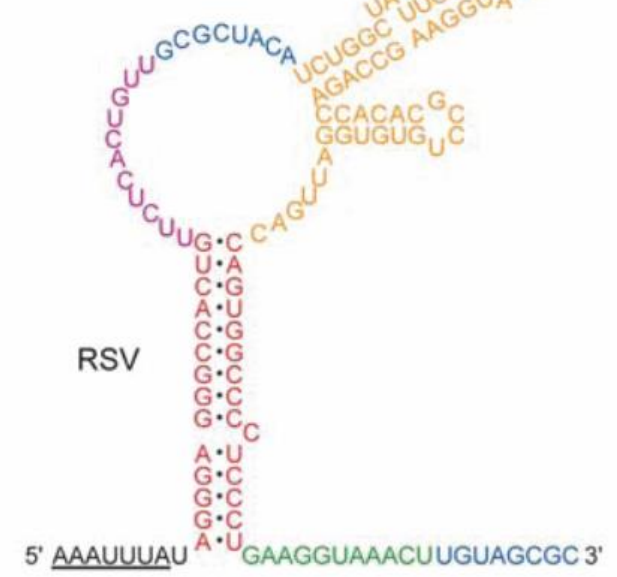

C
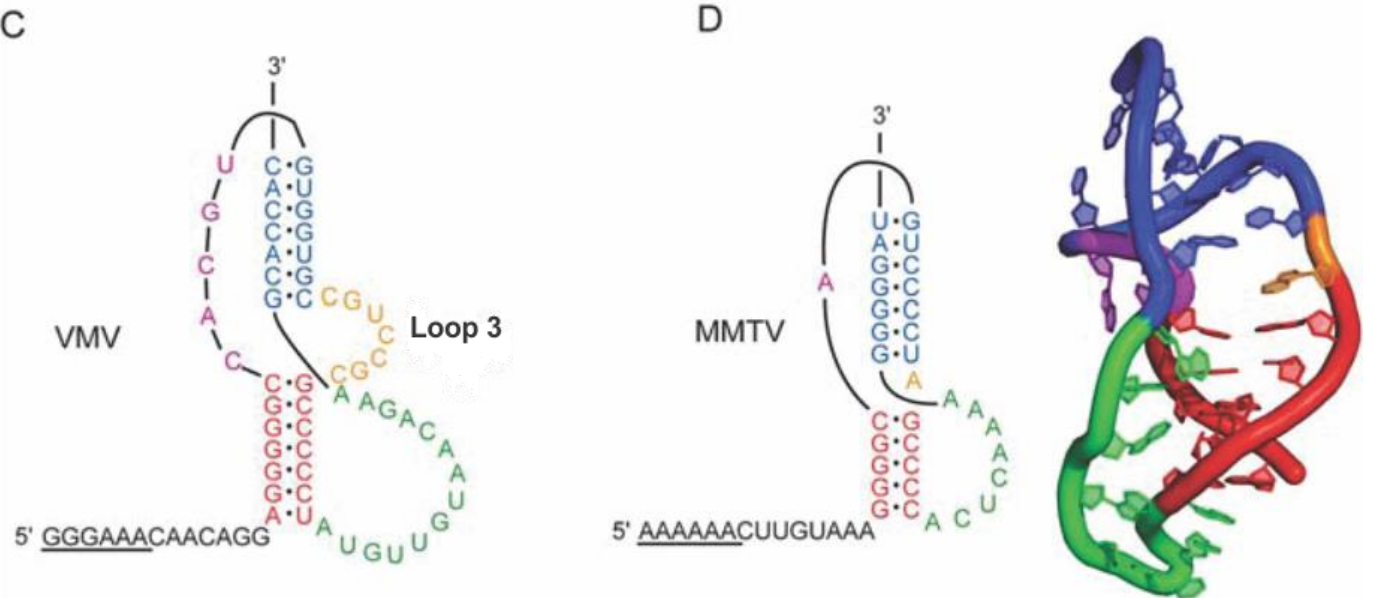

$E$

$\mathrm{F}$
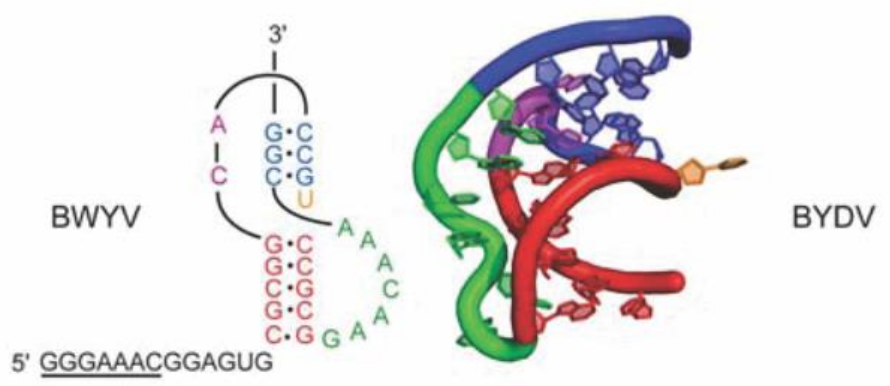

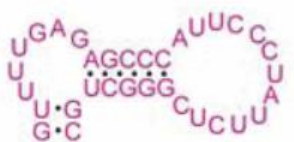

5' GGGUUUUUAGAG

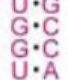

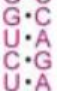

$U: G$
$C \cdot G_{A}$

C.GU
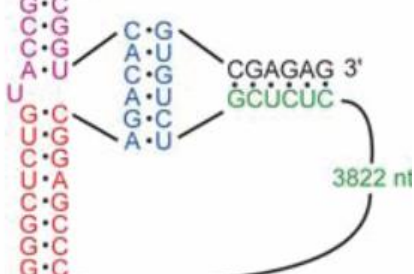

$\mathrm{G}^{\mathrm{G}}$

Figure 3. Examples of frameshifting sites found in viral sequences. Slippery sequences are underlined, pseudoknot elements stem1 (red), stem2 (blue), loop 1 (purple), loop 2 (green), loop3 (orange) are shown as ribbon models. Small arrow in $(A)$ indicates the place of helical junction where loop 3 is located in some pseudoknots. (A) Infectious bronchitis virus, (B) Raus sarcoma virus (RSV), (C) Visna-Maedi virus (VMV), (D) mouse mammary tumour virus gag/pro (MMTV), (E) beet western yellow virus (BWYV) and (F) barley yellow dwarf virus (BYDV). Designation of L2-L3 is reversed (modified from Brierley et al., 2010). 
The relationship between the mechanical properties of a pseudoknot and frameshifting was investigated by many groups. Hansen et al. performed experiments with optical tweezers where unfolding forces were measured for various frameshifting pseudoknots. They demonstrated that the most efficient frameshifting pseudoknot required the highest unfolding force thereby correlated the frameshifting efficiency with the pseudoknot strength (Hansen et al., 2007). In another study Green et al., using optical tweezers showed that the IBV pseudoknot requires higher force to unfold than for simpler hairpins. They suggested that frameshifting depends on the difficulty of unfolding the mRNA. On the other hand, no strong correlation was observed between frameshifting efficiency and the mechanical properties of various mRNA structures used (Green et al., 2008). Recently it has been reported that not the resistance to unfolding, but the conformational plasticity of the pseudoknot (i.e. its ability to form alternate pseudoknot structures) correlates with frameshifting efficiency (Ritchie et al., 2012).

Another determinant of frameshifting is the distance between the slippery site and secondary structure. Mutational studies suggested that spacer length of less than 5 nucleotides impairs the efficiency of frameshifting significantly, while 7-8 nucleotide distance between the slippery site and the pseudoknot is optimal (Brierley et al., 1992; Napthine et al., 1999). The last element in the mRNA that influences frameshifting efficiency is the presence of SD-like sequence upstream of the frameshifting site. In bacteria, the SD element can act as a frameshifting stimulator as reported for the dnaX gene (Larsen et al., 1997; Larsen et al., 1994), and in IS911 (Prere et al., 2011). Both of them have a putative SD sequence, GAAGX ( $\mathrm{X}=\mathrm{C}$ or $\mathrm{A}$ ), 10-11 nucleotides upstream of the P-site codon which is the first slippery triplet of the slippery sequence (Fayet and Prère, 2010). Short spacing between the SD-like sequence and the P-site codon can destabilize base pairing of peptidyl-tRNA and thereby effect the maintenance of the reading frame (Devaraj and Fredrick, 2010).

\subsection{Models of programmed ribosomal frameshifting}

Mutational studies performed at the frameshift sites generally agree on a strict requirement for stimulatory elements. Indeed frameshifting can possibly take place at every phase of the translation elongation cycle (Figure 4). Although first examples of ribosomal -1 frameshifting 
were published in the eighties (Jacks et al., 1988a), detailed information on mechanisms of reading frame maintenance and programmed frameshifting events is still incomplete.

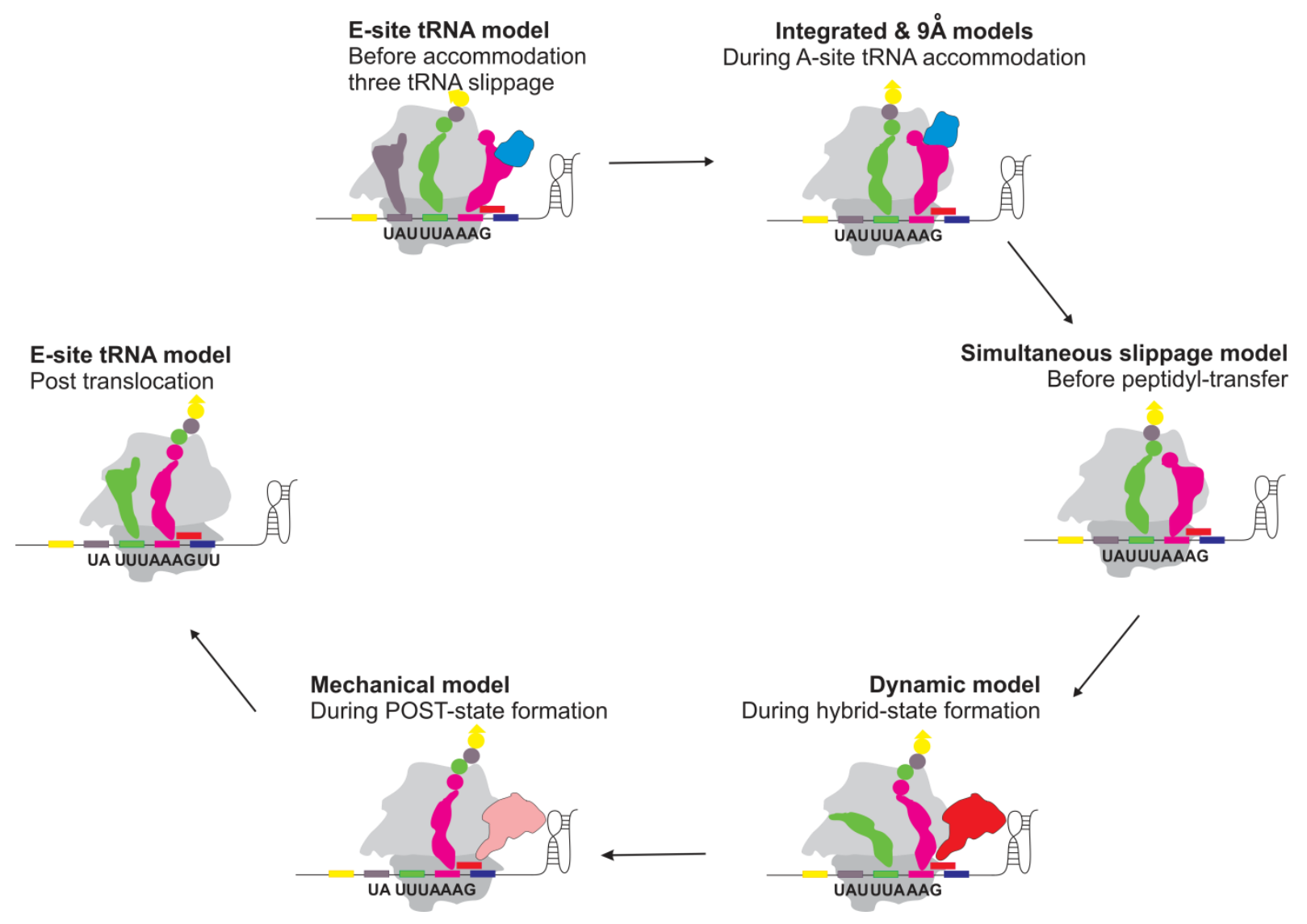

Figure 4. Proposed models of $\mathbf{- 1}$ frameshifting. Representation of the models each taking place at different phases of the translation elongation cycle. IBV frameshifting site is depicted for relevance with the current study. See text for details of each model (Modified from: Brierley et al., 2010).

Several questions should be answered to determine the precise timing and mechanism of frameshifting. During which stage of translation elongation is the codon anticodon base pair interaction between the mRNA and tRNA disrupted? Does competition at the frameshift site during decoding play a role on efficiency of frameshifting? Does EF-G remain bound longer on the ribosomes during - 1 frameshifting? Those lead to the big question, what are the factors that overcome the accuracy of reading frame maintenance in the ribosome? Based on the genetic, structural and mutational studies several models have been published. This section will cover the proposed mechanisms of -1 frameshifting (Figure 4). 


\subsubsection{Integrated model and 9 Å model}

It was proposed that frameshifting would occur during the accommodation of the A-site tRNA in the integrated model of frameshifting (Harger et al., 2002). The integrated model combined mutagenesis analysis with antibiotic probing of effects on frameshifting. Antibiotics that prevent accommodation, such as anisomycin, decrease -1 frameshifting. Another drug, sparsomycin, which causes the ribosomes to spend a longer time at the $A$ and P sites, leads to -1 frameshifting (Dinman et al., 1997). Later, it was shown that mutants of ribosomal protein L3 that cause slower peptidyl transfer induce -1 frameshifting (Meskauskas et al., 2003; Peltz et al., 1999). Based on such observations, it was concluded that post-peptidyl transfer ribosomes cannot slip and -1 frameshifting was most likely before peptidyl transfer (Harger et al., 2002).

One modification of the integrated model was the so called $9 \AA$ model, which brought a mechanical explanation to -1 frameshifting during $A$ site aa-tRNA accommodation (Plant, 2003). The model was based on the $9 \AA$ movement of the anticodon of the A site tRNA upon accommodation that was predicted from modeling data by Noller et al. (Noller et al., 2002). Plant et al. proposed when the movement of mRNA is blocked by a downstream cis-acting element, such as the pseudoknot, the anticodon at the A site may be pulled by one base. As a result of this movement, the mRNA would be stretched at the region between the pseudoknot and the anticodon occupying the $A$ site. The tension created at the pseudoknot would then be relieved by uncoupling of codon-anticodon interactions at the A and P sites. This uncoupling was suggested to allow the movement of mRNA by one base towards the $5^{\prime}$ end (Plant, 2003).

\subsubsection{Simultaneous slippage model}

The first model of frameshifting was proposed by Jacks et al. based on mutation studies in the RSV (Jacks et al., 1988a). The model requires tandem slippage of two tRNAs from zero frame $(X)-X X X-Y Y Y$ codons to -1 frame $X X X-X Y Y-(Y)$ codons at the slippery heptamer. Slippage is possible, because after simultaneous slippage one nucleotide to the $5^{\prime}$, tRNAs can base pair with two out of three nucleotides, maintaining near-cognate base pairing interactions in the new reading frame. In this model, peptide bond formation and translocation was 
proposed to occur after frameshifting, however the peptide sequence analysis is in agreement with both pre- and post-peptidyl tRNA slippage mechanisms. It is not clear how the tandem slippage of tRNAs would occur, considering the kink of mRNA between the $A$ and the P sites (Yusupov et al., 2001).

\subsubsection{Tortional restraints model}

Tortional restraints model is complementary to the $9 \AA$ model to explain how pseudoknots cause frameshifting (Plant, 2003; Plant and Dinman, 2005). The combination of the two models was proposed to explain mechanistically the original simultaneous slippage model (Jacks et al., 1988a). The tortional restraints model stated that the restraints at the stem 2 of the pseudoknot act against the intrinsic helicase activity of the ribosomes. The pause generated during the unfolding of the pseudoknot specifically positions the $A$ and $P$ sites on the heptameric slippery site, thereby stimulating -1 frameshifting. This model relates the efficiency of frameshifting to the degree of rotational freedom and the resistance of the secondary structure to unfolding forces.

\subsubsection{Dynamic model}

The dynamic model was first proposed by Weiss et al. and suggested that tRNA slippage occurs during the formation of the hybrid states, or during the translocation event itself (Weiss et al., 1989). In another study, it was reported that the movement of the tRNA to the $\mathrm{P} / \mathrm{E}$ state destabilizes the codon-anticodon base pair interactions and cause frameshifting (Spiegel et al., 2007). It was also suggested, because some tRNAs favor hybrid states of the ribosome more than others, that the tendency of tRNAs to slip would vary (Giedroc and Cornish, 2009). Thus, according to this model, frameshifting could be promoted by EF-G binding to the hybrid state ribosomes through stabilization of the P/E state (Brierley et al., 2010).

\subsubsection{Mechanical model}

The mechanical model was proposed by Namy and colleagues (Namy et al., 2004) based on the cryo-electron reconstruction of the $80 \mathrm{~S}$ ribosome in complex with the IBV frameshifting pseudoknot stalled with cyclohexamide. Due to the relatively low resolution (16 $⿱$ A), the 
authors could not visualize the mRNA stalled at the entry channel. It had depicted putative ribosomal helicase proteins $\mathrm{rpS} 2$, rpS3 and rpS9 at the mRNA entry channel. eEF-2 (the homolog of the prokaryotic EF-G) was observed to be trapped at the A site, while the P-site tRNA was not correctly positioned to have the right codon anticodon interactions; therefore, it was suggested to attain a distorted state $A / P^{\prime}$. The bent in the tRNA structure would direct the anticodon arm of the tRNA towards the A site which is occupied by eEF-2, while the T loop of the tRNA is pushed upwards. Based on this, a model was proposed in which the ribosome is unable to move on the mRNA in the $3^{\prime}$ direction due to the pseudoknot structure. Because the tRNA is bound to the mRNA by anticodon codon base pairing, eEF-2 action for translocation builds tension on the tRNA. As a result of the opposing forces exerted by the ribosome and the eEF-2, the tRNA at the P site confers the bent structure. The tension on the tRNA is relieved by breakage of the codon anticodon interactions subsequently followed by re-pairing with the mRNA in the new -1 frame.

\subsubsection{E-site tRNA models}

Several studies pointed out the importance of the $E$ site in translational fidelity and reading frame maintenance (Marquez et al., 2004; Nierhaus, 2006; Sanders and Curran, 2007). In addition, the SD interaction with the $3^{\prime}$ end of the $16 \mathrm{~S}$ rRNA is believed to destabilize the Esite tRNA and have importance in frameshifting (Marquez et al., 2004). There are two models which put particular emphasis on the E-site during frameshifting; the posttranslocation model (Horsfield et al., 1995) and the three-tRNA model (Leger et al., 2007).

The P/E state post-translocation model had been proposed by Horsfield et al. in which shift of the reading frame takes place when the tRNA is in P/E state (Horsfield et al., 1995). They found that the mutations at the codon immediately $3^{\prime}$ of the slippery site to a stop codon drastically induced frameshifting. They interpreted that the zero-frame codon is displayed at the A site during frameshifting. The authors suggested that frameshifting occurs after the peptidyl transfer and the translocation at the slippery site but before incorporation of the next aminoacyl-tRNA (Horsfield et al., 1995).

Léger et al. performed mutational studies in the HIV frameshifting site and reported an extended 10 nucleotide long slippery site was involved in -1 frameshifting rather than the 
heptanucleotide motif (Leger et al., 2007). Mutations at the E site were found to influence frameshifting in case of HIV. In their proposed model, during translocation the mRNA moves by two nucleotides instead of three nucleotides such that the tRNAs occupy an intermediate state with $\mathrm{P}$ and $\mathrm{E}$ sites, imperfectly positioned in a $E^{*} / \mathrm{E}^{*}$ and $\mathrm{P}^{*} / \mathrm{P}^{*}$ states (Pan et al., 2007). Because the $A$ site is vacant at this time point, the next accommodation stage can start with the binding of aminoacyl tRNA to the $A / T^{*}$ site, which also differs from the classical entry site. The tRNAs base pair in the new reading frame as they cannot base pair in the correct frame. At this post-translocation stage the slippage of tRNAs take place subsequently. This model mentioned two pauses during this process, first one when the translocation is blocked and the second one when the tRNAs shift to the new reading frame. The three-site tRNA model of the ribosome is contradicted by biochemical work (Petropoulos and Green, 2012; Semenkov et al., 1996) and with the results of single molecule analysis that showed that a three tRNA-bound state is almost never observed during translation (Uemura et al., 2010). These finding challenges the three-site tRNA binding model proposed for frameshifting; however, it is still possible that the E site plays a role under unusual circumstances like -1 frameshifting. 


\subsection{Aim of the thesis}

The -1 programmed frameshifting models proposed so far agree that frameshifting is taking place during the elongation cycle, however, it is not clear at which step it occurs, e.g. before or after the peptidyl transferase reaction or during translocation. The aim of this work was to determine the mechanism of -1 frameshifting by dissecting steps of elongation cycle in a kinetic framework. Here, we employed in vivo as well as rapid kinetic methods at the minimal IBV $1 a / 1 b$ frameshift site to examine frameshifting in $E$. coli $70 S$ ribosomes. Using radioactive or fluorescent observables in rapid kinetic experiments, we were able to track individual steps of elongation in a codon resolved manner. Some of the questions we desired to answer were: (i) At which step of translation elongation does frameshifting occur? (ii) What are the effects of individual stimulatory elements on the minimal frameshifting system employed? (iii) Is EF-G present on the ribosomes during frameshifting? 


\section{RESULTS}

\subsection{IBV $1 a / 1 b$ as a model system to study frameshifting in vitro}

To study the mechanism of programmed ribosomal frameshifting, we have chosen a minimal IBV $1 a / 1 b$ construct, for which significant levels of -1 frameshifting had been demonstrated (Brierley et al., 1989; Brierley et al., 1992; Brierley et al., 1997; Brierley et al., 1991; Napthine et al., 2003). To study frameshifting in vitro, we utilized a highly purified translation system consisting of $70 \mathrm{~S}$ ribosomes, initiation factors, aminoacyl-tRNAs and elongation factors (EF-G and EF-Tu) from E. coli and monitored frameshifting in real time using rapid kinetic methods.

In order to be translated in the $E$. coli system, the minimal IBV $1 a / 1 b$ frameshifting mRNA was constructed in a following way (Figure 5). It contained (1) a SD sequence and an initiation codon upstream of the recoding site, (2) the slippery site and (3) the pseudoknot. The SD sequence was necessary to initiate translation. The SD sequence is seven nucleotides upstream of the AUG start codon, which ensured initiation efficiencies of the model mRNAs close to $100 \%$. The distance between the initiation codon and the slippery site was two nucleotides, which should ensure that the SD-aSD interactions are weakened or resolved at the time of the slippage. In the original IBV $1 a / 1 b$ slippery sequence (U UUA $\underline{A A C}$ ), the second codon ( $A A C$ ) encoding for Arg was shown to lead to less efficient frameshifting in $E$. coli (Brierley et al., 1997). To increase the frameshifting efficiency, this codon was modified to AAG, which encodes for Lys (Napthine et al., 2003). Our minimal IBV $1 a / 1 b$ fragment encodes for MetTyrLeuLysPhe (MYLKF), with the slippery site U $\underline{\text { UUA }} \underline{\mathrm{AAG}}$ corresponding to the third and fourth codons Leu and Lys, respectively. At the overlapping codon $3^{\prime}$ of the slippery site, the next amino acid incorporated after the tetrapeptide (fMYLK) would be Phe in case of the readthrough and a Val in case of -1 frameshifting. Finally, the pseudoknot was a modified variant of the IBV pseudoknot with a loop 2 that was shortened from 35 nucleotides to 8 nucleotides (Brierley et al., 1992; Brierley et al., 1991; Napthine et al., 2003; Somogyi et al., 1993). This minimal IBV pseudoknot is six nucleotides downstream of the slippery site in order to ensure efficient frameshifting as shown previously (Brierley et al., 1992; Napthine et al., 1999). 


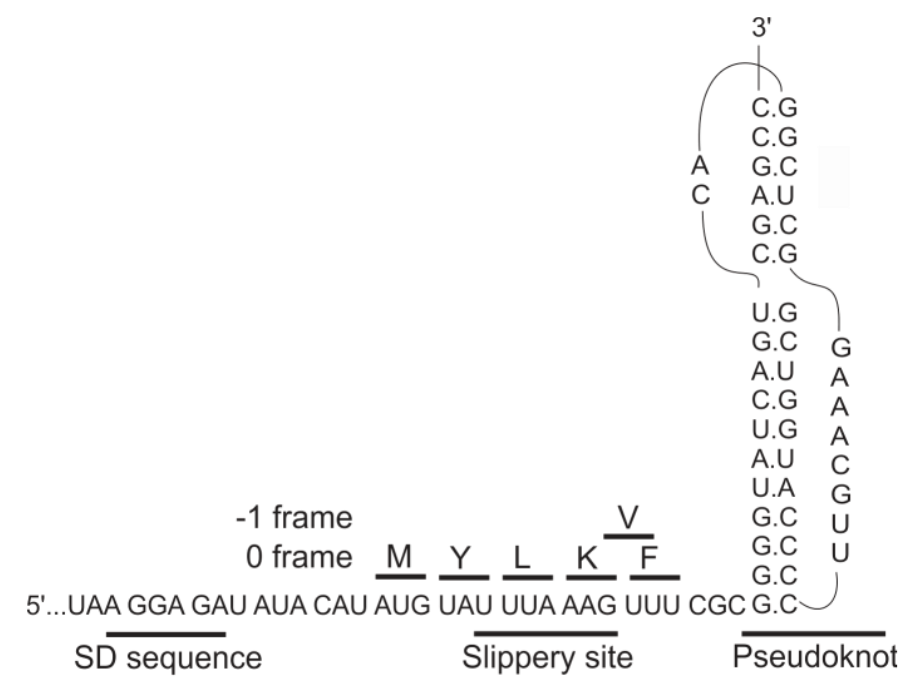

Figure 5. Design of frameshifting mRNA. mRNAs for in vitro translation contained a modified IBV $1 a / 1 b$ fragment. The encoded amino acids in zero and -1 frame are indicated above the nucleotide sequence. As a result of -1 frameshifting Val is the first out-of-frame amino acid added to the peptide chain.

In addition to the -1 frameshifting mRNA, a series of model mRNAs were generated based on the minimal IBV $1 a / 1 b$ frameshifting construct $(+/+)$ which differed in their frameshifting stimulatory elements: a control mRNA that lacks both of the stimulatory elements (-/-), an mRNA without a pseudoknot (+/-), and an mRNA without a slippery site (-/+). Additionally, a control mRNA (-/-) that encodes Val in zero frame instead of Phe was synthesized to evaluate decoding of Val in zero frame. In control mRNAs (-/- and -/+) the slippery site (U UUA AAG) was disrupted by a point mutation at the UUA codon to UUG. Importantly, both codons were still recognized by the same isoacceptor tRNA ${ }^{\text {Leu5 }}$ (UUA/G). Therefore, the amino acid sequences of the peptides synthesized remained unchanged. This allowed us to study frameshifting on a sequence where a slippery site is disrupted without changing the identity of tRNAs. Another model mRNA was constructed based on the mRNA without a slippery site or a pseudoknot (-/-) that enabled us to study differences during decoding of the AAA instead of AAG codon by Lys-tRNA ${ }^{\text {Lys }}$.

\subsection{Functionality of the IBV $1 a / 1 b$ fragment in vivo}

As a first step in utilizing a viral mRNA fragment in the bacterial translation system, we needed to validate translation of the minimal IBV $1 a / 1 b$ frameshifting site in $E$. coli. We have developed an in vivo assay in which the minimal IBV $1 a / 1 b$ gene fragment was cloned into a 
dual luciferase reporter construct containing firefly and renilla luciferases (Figure 6A). The dual luciferase construct was designed such that the translation of renilla luciferase was a measure of efficiency of read-through translation or -1 frameshifting of the inserted fragment. In order to report i.e. frameshifting, the renilla luciferase gene was positioned in the -1 reading frame. Full-length firefly-renilla fusion protein was expressed only if the ribosomes would shift to the -1 frame while translating the frameshift site-containing linker. The firefly luciferase gene was used as an internal reference to compare efficiencies of inframe translation from each expression vector relative to each other.

A

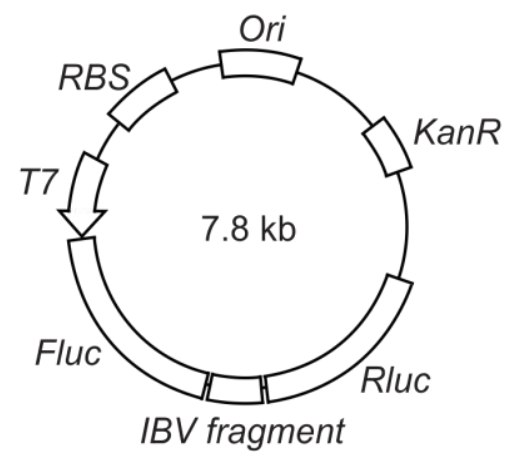

B

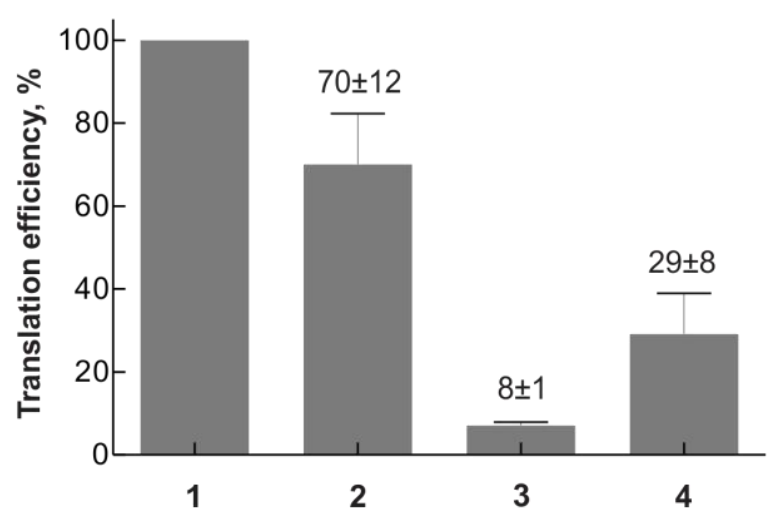

Figure 6. In vivo dual luciferase assay in E. coli. (A) A fusion protein of firefly and renilla luciferases linked by a fragment of frameshifting site of IBV $1 a / 1 b$ gene was used to study frameshifting in vivo. The complete fusion protein Fluc-Rluc was produced only when the linker region was translated due to frameshifting. (B) Efficiencies of translation were determined by measuring the enzymatic activities of the two luciferases. Briefly, the activity ratio of renilla to firefly luciferase of test constructs was divided by the activity ratio of the control reporter, multiplied by 100 (Grentzmann et al., 1998a). Efficiency of translation is plotted for control (-/-/0 frame R/uc) (1), frameshifting (+/+/-1 frame R/uc) (2), read-through $(+/+/ 0$ frame $R /$ luc) (3) and minus pseudoknot (+/-/-1 frame R/uc) (4) constructs. Experiments were repeated at least 3 times for error calculations.

The linear working range of the assay was determined by measuring the relative activity ratios of renilla to firefly luciferases for different samples over time. The measurements were within the linear working range of the assay (5-15 min). Nevertheless, to ensure reproducible results concerning the sensitivity and decay of the assay substrates, it was critical that all the luminescence measurements were done at the same time points for different vectors utilized. 
Using the in vivo dual luciferase assay, we have measured about $70 \%$ frameshifting in the construct containing the minimal IBV $1 a / 1 b$ fragment with a slippery site and a pseudoknot $(+/+/-1$ frame $R / u c)$. In the zero reading frame (+/+/0 frame $R / u c)$ the efficiency of translation was only $8 \%$, giving a frameshift to non-shift ratio of 9:1 for the IBV $1 a / 1 b$ fragment. Taken together, these results indicated a clear preference of frameshifting over zero-frame readthrough during translation of the minimal IBV $1 a / 1 b$ fragment. In $E$. coli previous studies reported an efficiency of IBV 1a/1b frameshifting of around 40\% (Brierley et al., 1997).

Bacterial and eukaryotic ribosomes were reported to have different requirements for frameshifting (Garcia et al., 1993; Napthine et al., 2003; Sung and Kang, 2003), i.e. IBV pseudoknot mutations might completely abolish frameshifting in rabbit reticulocyte lysate (Brierley et al., 1991). In E. coli the requirement for a pseudoknot is not conserved; a stem loop can also stimulate frameshifting in IBV $1 a / 1 b$, however deletion of the downstream secondary structure was reported to decrease frameshifting to $2 \%$ (Brierley et al., 1997). In order to check the stimulatory element requirements in $E$. coli, we deleted the pseudoknot fragment of the frameshifting construct, while the rest of the sequence was conserved. In the absence of the pseudoknot (+/-/-1 frame R/uc), the efficiency of frameshifting was decreased to $29 \%$. This showed that in our E. coli system in the presence of the slippery site, frameshifting takes place despite the lack of the stimulatory secondary structure albeit with lower efficiency (Figure 6B).

\subsection{Decoding at the frameshifting site}

Once we established that -1 frameshifting on the IBV $1 a / 1 b$ fragment takes place with high efficiency in E. coli, we moved on to examine the kinetics of the event in vitro using the reconstituted translation system. In order to determine at which point during translation elongation frameshifting takes place, we have initially followed the decoding of consecutive codons. Due to the transient nature of these reactions in the ribosome, we have employed the rapid-quench-flow. Rapid kinetic measurements utilizing radioactively-labeled amino acids allowed us to identify the translation intermediates, and determine the kinetic rate constants of their formation and consumption in subsequent incorporation reactions. 
The purified components of the in vitro translation system include purified ribosomes, initiation factors (IF1, 2, 3), aminoacyl-tRNAs and elongation factors (EF-Tu and EF-G). The experiments were carried out under pseudo-first order conditions; for that we used an excess of ternary complexes and EF-G over initiation complexes.

To monitor amino acid incorporation on consecutive codons, 70S initiation complex with $\mathrm{fMet} \mathrm{tRNA} \mathrm{fMet}^{\mathrm{f}}$ at the $\mathrm{P}$ site was rapidly mixed with excess of different ternary complexes EFTu.GTP.aminoacyl-tRNA (Tyr, Leu, Lys, Phe and Val) in the presence of EF-G (Figure 7A). After a desired time over a logarithmic time scale the reactions were quenched with a strong base $(\mathrm{KOH})$, which resulted in an instantaneous stop of the reaction, chemical degradation of the RNA and release of the synthesized peptide.

To analyze the peptide composition in each sample over the time course, reversed-phase HPLC was performed. Chromatography conditions were optimized to allow for a robust separation of peptide products of different size and composition up to penta- (fMYLKF/V) peptides (Figure 7B-C) (Guo et al., 1986a; Guo et al., 1986b). In the optimization process, we performed translation of peptides of different lengths which contain an $\left.\mathrm{f}^{3} \mathrm{H}\right]$ Met at the $\mathrm{N}$ terminus and $\left[{ }^{14} \mathrm{C}\right]$-labeled amino acid at the C-terminus. Subsequently, di-, tri-, tetra- and penta- peptides with $\mathrm{C}$-terminal $\left[{ }^{14} \mathrm{C}\right]$-labels were analyzed chromatographically and the retention times of the corresponding peptides were determined (Figure 7B-C). In reversedphase HPLC, the retention times of a peptide depend mainly on the hydrophobicity and to some extend on the molecular weight. Peptide chain length is especially critical for peptides longer than 20 amino acids (Guo et al., 1986b). Because the hydrophobicity of tripeptides ( $\mathrm{FMYL}$ ) and tetrapeptides ( $\mathrm{fMYLK}$ ) differed significantly, tripeptides ( $\mathrm{fMYL}$ ) eluted later than the more hydrophilic tetrapeptides (fMYLK) (18 $\mathrm{min}$ and $16 \mathrm{~min}$, respectively). The fMYLKF peptides were more hydrophobic than the fMYLKV and had therefore longer retention times. 
A

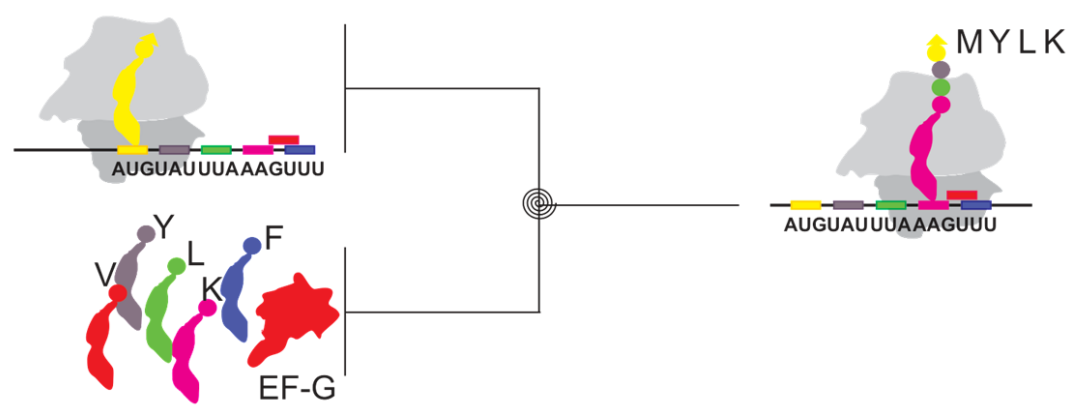

B

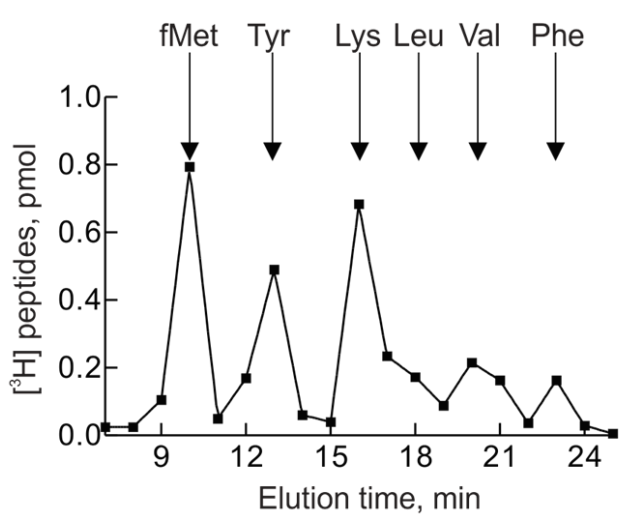

C

\begin{tabular}{lc}
\hline Peptide sequence & Elution time, min $( \pm 1)$ \\
\hline fMet & 10 \\
fMet-Tyr & 13 \\
fMet-Tyr-Leu & 16 \\
fMet-Tyr- Leu-Lys & 23 \\
fMet-Tyr-Leu-Lys-Phe & 20 \\
fMet-Tyr-Leu-Lys-Val & \\
\hline
\end{tabular}

Figure 7. In vitro translation and analysis of peptide products. (A) Experimental setup of in vitro translation. Amino acid incorporation was monitored by rapid mixing of purified initiation complexes 70S.fMet-tRNA ${ }^{\mathrm{fMet}}$.mRNA $(0.2 \mu \mathrm{M})$ with ternary complexes EF-Tu.GTP-aminoacyltRNA for Tyr, Leu, Lys, Phe and Val (1.5 $\mu \mathrm{M}$ each) in the presence of saturating amounts of EF$\mathrm{G}(2 \mu \mathrm{M})$, in a quench-flow apparatus. (B) Translation products at each time point (depicted in squares connected with lines) were collected and separated via reversed-phase HPLC using a linear $0-65 \%$ acetonitrile gradient. As an example, the retention profile and amount of peptide products at $0.7 \mathrm{~s}$ of translation of mRNA (+/-) is given. (C) The order of chromatographic elution of the translation products synthesized by in vitro translation.

Once the chromatography conditions were established, for the rest of the experiments, radioactively labeled amino acids $\mathrm{f}\left[{ }^{3} \mathrm{H}\right]$ Met and $\left[{ }^{14} \mathrm{C}\right]$ Lys allowed identification and calculation of the amount of peptides at the corresponding peak on the chromatograms using double scintillation counting. As expected, each initiated $70 \mathrm{~S}$ ribosome would carry the $\mathrm{f}\left[{ }^{3} \mathrm{H}\right] \mathrm{Met}$, whereas only those that translated up to tetrapeptides ( $F M Y L K$ ) and pentapeptides (fMYLKF and fMYLKV) would contain the dual $f\left[{ }^{3} \mathrm{H}\right]$ Met and $\left[{ }^{14} \mathrm{C}\right]$ Lys labels. This approach allowed us to selectively monitor the rate of incorporation of each amino acid to the growing peptide chain (Figure 7B). 
When peptide products were analyzed after different incubation times (i.e. from 0.02-15 s); we could observe the appearance and disappearance of reaction products during translation. In order to evaluate this multi-step kinetic mechanism, a mathematical model was developed describing the kinetics of sequential amino acid incorporation through the course of translation. This model was built in the form of a reaction scheme that allows discrimination of each incorporation step and corresponding rate constants. The model simulation involved calculations of the fractions of di- (fMY), tri- (fMYL), tetra- (fMYLK) and penta- (fMYLKF/V) $\left[{ }^{3} \mathrm{H}\right]$-labeled peptides over total $\left[{ }^{3} \mathrm{H}\right]$-labeled peptides as a function of time which was plotted separately for every peptide observed (as in Figure 8A-C-E). The basic kinetic scheme of the multi-step amino acid incorporation was as follows:

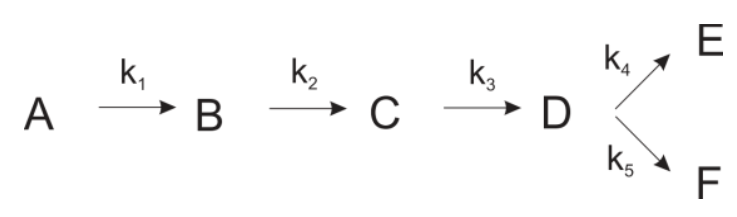

Initiation complexes with $\mathrm{fMet}_{\mathrm{N}} \mathrm{tRNA} \mathrm{f}^{\mathrm{fMet}}$ at the $\mathrm{P}$ site $(\mathrm{A})$ could accommodate Tyr-tRNA ${ }^{\mathrm{Tyr}}$ forming dipeptides (fMY) (B). Translocation of the fMetTyr-tRNA ${ }^{\text {Tyr }}$ would be followed by the next elongation cycle forming tripeptides ( $F M Y L)(C)$ and so on. At the last step, either a Val (E) or Phe (F) can be incorporated to the tetrapeptide ( $f M Y L K$ ) chain; therefore this step was modeled in a branched manner.

Additionally, at every elongation cycle a certain population of ribosomes ( $10 \%)$ aborted translation; therefore non-reactive product $(\mathrm{Xb})$ in each step was subtracted in the rate calculations. For each incorporation step the differential model equations were characterized by the apparent rate constants ( $k_{x}$ where $\left.X=1-5\right)$ and the concentrations (in the form of $\mathrm{X}-\mathrm{Xb})$ for the respective peptide (Methods). Solving the model equations involved least squares data fitting using numerical integration. 


\subsubsection{Optimization of in vitro translation}

As a first step in testing decoding of the IBV $1 a / 1 b$ fragment in vitro, ternary complexes were titrated over the initiation complexes to ensure that the amino acid incorporation at the frameshift site was not rate-limited by the preceding amino acid incorporation events. For the codons before and the frameshifting site (UAU, UUA/G and AAG), increasing amounts of ternary complexes EF-Tu.GTP.Tyr-tRNA ${ }^{\text {Tyr }}$, EF-Tu.GTP.Leu-tRNA ${ }^{\text {Leu }}$ or EF-Tu.GTP.Lys-tRNA ${ }^{\text {Lys }}$ were titrated over purified $70 \mathrm{~S}$ initiation complexes. Peptide intermediates at each time point (depicted in symbols in Figure 8A, C, E) were separated by HPLC and their concentrations were evaluated as a function of time. The apparent rate constants of amino acid incorporation on Tyr, Leu and Lys codons were calculated using the mathematical model described above. The corresponding rate constants $\left(k_{1}, k_{2}, k_{3}\right)$ were measured at various ternary complex concentrations and were fitted by a hyperbolic function consistent with a concentration dependence of a monomolecular rearrangement (Figure 8B, D, F).

Ribosome complexes programmed with control mRNA (-/-) were titrated at the second codon, Tyr, where UAU codon was exposed at the A site. Increasing amounts of ternary complex EF-Tu.GTP.Tyr-tRNA ${ }^{\text {Tyr }}$ were added to initiation complexes on a time course and the formation of dipeptides ( $\mathrm{fMY}$ ) was monitored. The concentration dependence of the apparent rate constant of Tyr incorporation was hyperbolic and reached saturation at $3 \mu \mathrm{M}$ of ternary complex with the rate of $14 \mathrm{~s}^{-1}$ (Figure 8B). In order to monitor the dependence of Leu incorporation at the third codon (UUG) on ternary complex concentration, EFTu.GTP.Leu-tRNA ${ }^{\text {Leu }}$ was titrated over the ribosomal complexes in the presence of EF-G, while the concentration of EF-Tu.GTP.Tyr-tRNA ${ }^{\text {Tyr }}$ was kept constant and saturating $(3 \mu \mathrm{M})$. Because of the saturating amounts of the first ternary complex EF-Tu.GTP.Tyr-tRNA ${ }^{\text {Tyr }}$ and the presence of EF-G in the reaction mix, all reactive ribosomes would have fMetTyr-tRNA ${ }^{T y r}$ at the P site and display the UUG codon in the A site. At saturating concentrations ( $0.75 \mu \mathrm{M})$ the rate of incorporation of Leu was about $2 \mathrm{~s}^{-1}$ (Figure 8D). 
A

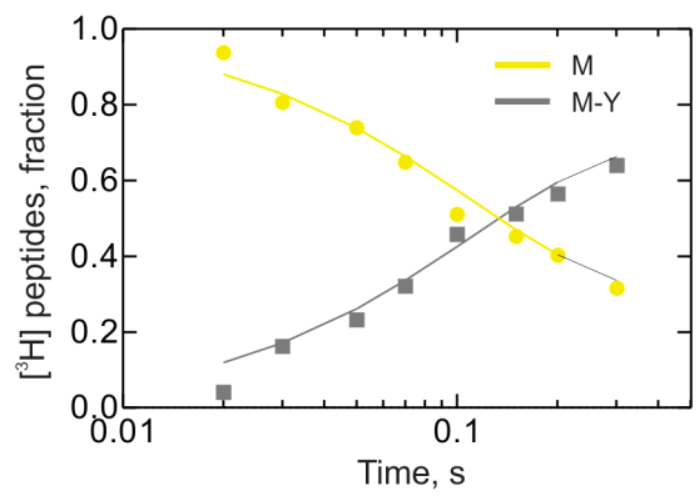

C

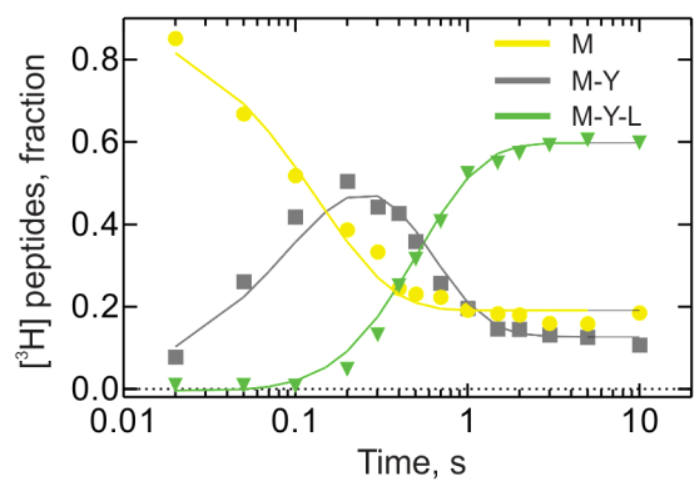

E

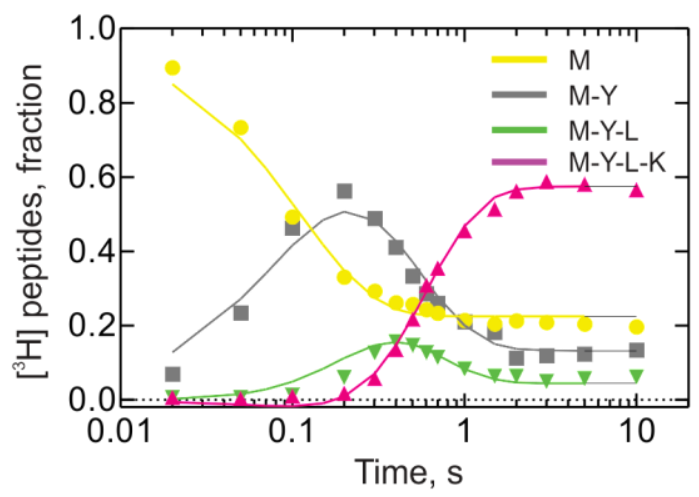

B

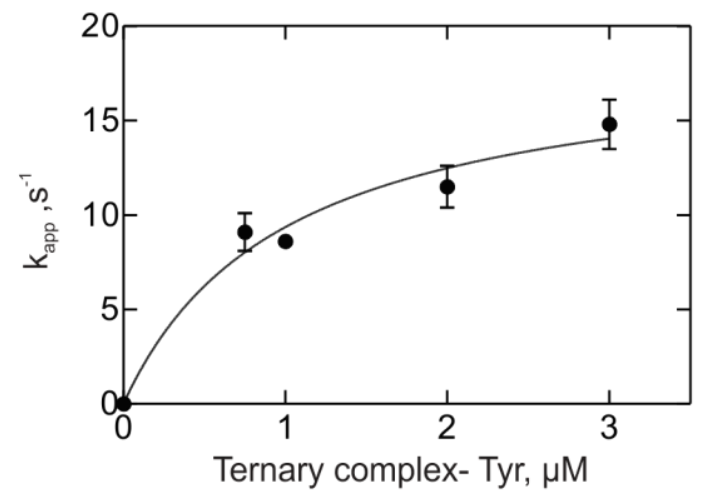

D

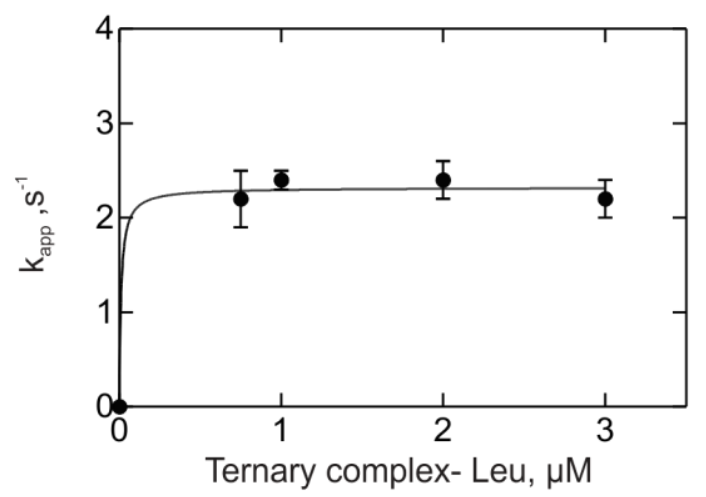

$\mathbf{F}$

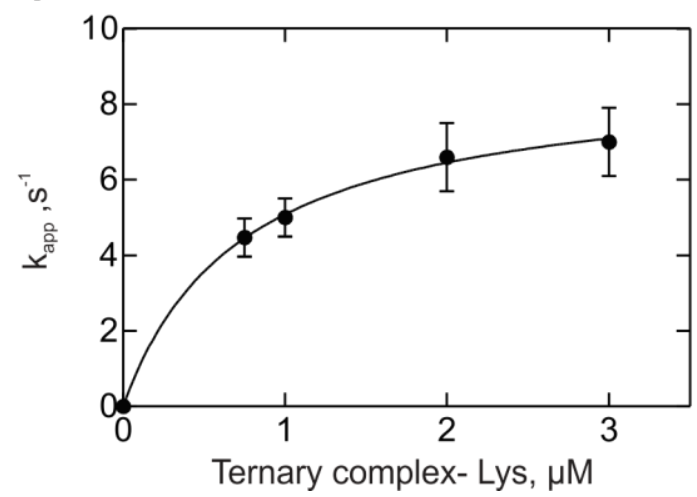

Figure 8. Dependence of peptide bond formation on ternary complex concentration. Concentrations of individual ternary complexes, EF-Tu.GTP-aminoacyl-tRNA for Tyr $(\mathbf{A}, \mathbf{B})$, Leu $(C, D)$ and Lys $(E, F)$, were increased in the presence of EF-G $(2 \mu M)$ over initiated 70 S ribosomes programmed with control mRNA (-/-) $(0.2 \mu \mathrm{M})$ and the time courses of formation of the respective peptides were measured in a quench-flow apparatus. Peptides were separated using reversed-phase HPLC by an acetonitrile gradient ranging from $0-65 \%$. Left panel: Time courses of synthesis of peptide products. Fraction of $\left[{ }^{3} \mathrm{H}\right]$ labeled peptides corresponding to $\mathrm{M}$ (yellow), MY (grey), MYL (green), MYLK (magenta) over total $\left[{ }^{3} \mathrm{H}\right]$ peptides is plotted. The symbols represent the calculated peptide fraction and the lines represent the fitted values calculated by the mathematical model using numerical integration. Right panel: The rates calculated for incorporation of each amino acid ( $Y, L$ and/or $K$ ) at increasing concentrations of ternary complexes EF-Tu.GTP.aminoacyl-tRNA. Lines represent the fit for each titration curve using a hyperbolic function. The rates at saturation for incorporation of Tyr $k_{a p p}=14 \mathrm{~s}^{-1}$ (B), Leu $k_{a p p}=2 s^{-1}(D)$ and Lys $k_{a p p}=7 s^{-1}(E)$. 
As described above, different Leu codons (UUA and UUG) were used in the frameshifting $(+/+)$ and minus pseudoknot (+/-) mRNAs, respectively, which were decoded by the same isoacceptor tRNA molecule (tRNA ${ }^{\text {Leu5 }}$ ). We tested decoding of UUA codon by tRNA ${ }^{\text {Leu5 }}$ using the ribosome complexes programmed with frameshifting mRNA (+/+) and mRNA without a pseudoknot (+/-). No significant differences were observed upon the incorporation of Leu during decoding of UUG or UUA codons (data not shown).

Finally, for the following codon (AAG) the effect of increasing ternary complex concentrations on the incorporation of Lys was monitored. 705 initiation complexes programmed with control mRNA (-/-) were rapidly reacted with saturating concentrations of EF-Tu.GTP.Tyr-tRNA ${ }^{\text {Tyr }}$ and EF-Tu.GTP.Leu-tRNA ${ }^{\text {Leu }}$ ternary complexes and increasing amounts of EF-Tu.GTP.Lys-tRNA ${ }^{\text {Lys }}$ ternary complex, in the presence of EF-G. The ternary complex EF-Tu.GTP.Lys-tRNA ${ }^{\text {Lys }}$ titration curve was hyperbolic and saturated at a concentration of $3 \mu \mathrm{M}$ with a rate of $7 \mathrm{~s}^{-1}$ (Figure $8 \mathrm{~F}$ ).

Next, we checked the dependence of rate of amino acid incorporation on the EF-G concentration. Initiation complexes programmed with frameshifting mRNA $(+/+)$ were rapidly reacted in the presence of saturating concentrations of ternary complexes, EFTu.GTP.aminoacyl-tRNA in the presence of increasing concentrations of EF-G. At each time point the collected peptide products were separated by reversed-phase HPLC and analyzed by radiaoctivity counting. The rates of incorporation of amino acids (Tyr, Leu and Lys) were not changed at various EF-G concentrations, showing that translocation is not rate limiting for the amino acid incorporation (Figure 9). 


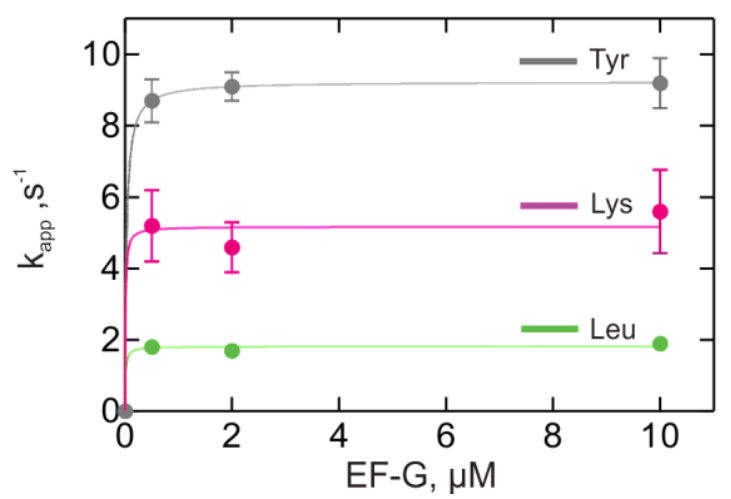

Figure 9. Dependence of amino acid incorporation on EF-G concentration. Formation of tetrapeptides ( $\mathrm{fMYLK}$ ) were monitored on ribosomal complexes programmed with frameshifting mRNA $(+/+)$ at varying concentrations of EF-G $(0.5 / 2 / 10 \mu \mathrm{M})$. Apparent rates of Tyr, Leu, and Lys incorporation were plotted against EF-G concentration.

Once the in vitro translation system was established and the analysis was optimized to monitor individual incorporation reactions, we focused on the fundamental question of decoding at the frameshifting site.

\subsubsection{Effect of frameshifting elements on decoding}

To monitor frameshifting, initiated $70 \mathrm{~S}$ ribosomes programmed with various model mRNAs (encoding for MetTyrLeuLysPhe or MetTyrLeuLysVal) were rapidly reacted with saturating concentration of the ternary complex mix with Tyr-, Leu-, Lys-, Val- and Phe-tRNAs in the presence of EF-G. Separation of the pentapeptides and analysis of rate of incorporation was done as described before.

Tyrosine is the first tRNA molecule binding at the vacant A site of the 70S initiated ribosomes in our system. In case of frameshifting mRNA (+/+), Tyr was incorporated at a rate $\left(9.1 \mathrm{~s}^{-1}\right)$ similar to the complexes programmed with control mRNA (-/-) $\left(8.4 \mathrm{~s}^{-1}\right)$. When the pseudoknot was removed (+/-) or the slippery site was mutated $(-/+)$ the rate was unchanged $\left(8.5 \mathrm{~s}^{-1}\right.$ and $8.0 \mathrm{~s}^{-1}$, respectively). Peptides fraction corresponding to dipeptides (fMY) were found after $0.05 \mathrm{~s}$ on and reached the maximum accumulation at $0.25 \mathrm{~s}$, followed by consumption in the following reaction (Figure 10). 
A 5' AUG UAU UUA AAG UUU CGC.......

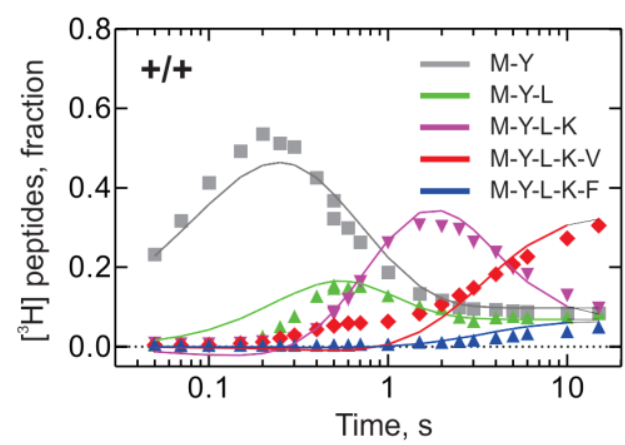

C 5' AUG UAU UUG AAG UUU CGC.......3'

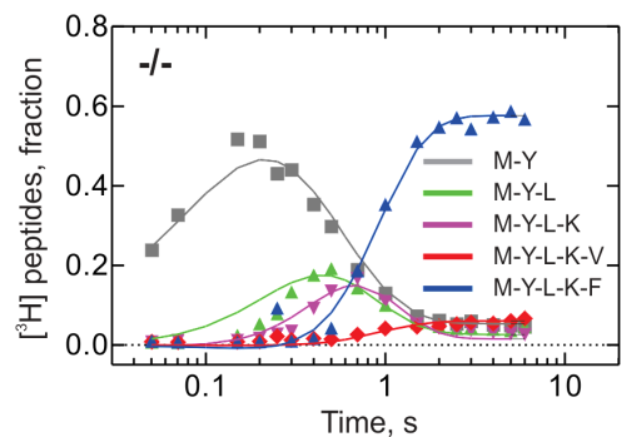

B 5' AUG UAU UUA AAG UUU CGC....... 3'

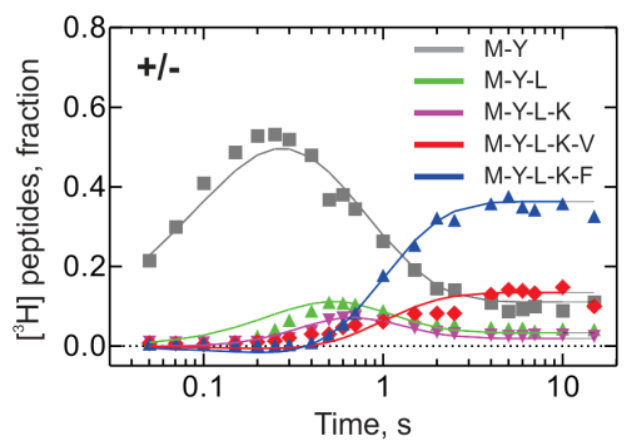

D 5' AUG UAU UUG AAG GUU UCG.......3'

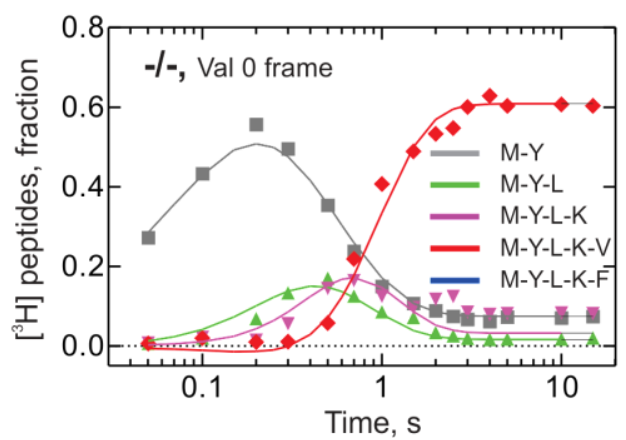

Figure 10. Kinetics of amino acid incorporation before and at the frameshifting site. (A-D) 705 initiation complexes programmed with different mRNA species $(0.2 \mu \mathrm{M})$ were rapidly reacted with a ternary complex mix containing EF-Tu.GTP.aminoacyl-tRNA (Tyr, Leu, Lys, Val, Phe) in the presence of EF-G ( $2 \mu \mathrm{M}$, in a quench-flow apparatus. The fraction of peptides ( $\left[{ }^{3} \mathrm{H}\right]$ peptide $/\left[{ }^{3} \mathrm{H}\right]$ total) at each time point corresponding to fMY (grey), fMYL (green), fMYLK (magenta), fMYLKV (red) and fMYLKF (blue) was used to calculate the rate of peptide bond formation by numerical integration. Fits yielding the apparent rate constants for the respective amino acid incorporation step are represented as continuous lines. Time course of translation intermediates produced by a complex programmed with a:

(A) frameshifting mRNA $(+/+)$.

(B) minus pseudoknot mRNA (+/-).

(C) minus slippery site, minus pseudoknot mRNA (-/-).

(D) minus slippery site, minus pseudoknot mRNA (-/-) that encodes Val in zero frame.

Similarly to Tyr, the ribosomes incorporated Leu forming the tripeptides ( $\mathrm{fMYL}$ ) with similar rates $\left(2.2 \mathrm{~s}^{-1}\right)$ independent of the type of mRNA used. Synthesis of tripeptides ( $\mathrm{fMYL}$ ) started after $0.15 \mathrm{~s}$ and reached maximum at $0.5 \mathrm{~s}$. Slight variations were observed in this rate for ribosome complexes programmed with no pseudoknot (+/-) mRNA $\left(1.4 \mathrm{~s}^{-1}\right)$ and with no slippery site $(-/+)$ mRNA $\left(2.9 \mathrm{~s}^{-1}\right)$. There was a delay during synthesis of tripeptides, which could not be fitted using the multistep incorporation model, leading to a difference between 
the simulated and experimental data. The variations in the apparent rate constants for Leu incorporation among different complexes could be related to this discrepancy (Figure 10).

In the minimal IBV $1 a / 1 b$ frameshifting fragment, AAG is the codon where slippage of the tRNA occurs (Brierley et al., 1997). Therefore, we aimed to identify whether -1 frameshifting would occur during the accommodation step of Lys as proposed previously (Harger et al., 2002; Plant, 2003). The formation of the tetrapeptides (fMYLK) was monitored on ribosomal complexes programmed with model frameshifting $(+/+)$ and various control mRNAs. In frameshifting $(+/+)$ complexes a remarkable difference in the tetrapeptide traces (shown in magenta in Figure 10A) was observed compared to the control (-/-) complexes in terms of the amplitude. In case of control (-/-) constructs synthesis of tetrapeptides started at $0.25 \mathrm{~s}$ and reached maximum level at $0.7 \mathrm{~s}$, whereas for the frameshifting (+/+) complexes the corresponding tetrapeptide peak position was shifted to $2 \mathrm{~s}$ (Figure 10C). Despite that amplitude difference, apparent rate of the Lys incorporation in frameshifting $(+/+)$ construct $\left(4.6 \mathrm{~s}^{-1}\right)$ was not changed (Table 1). Rather, the difference in the amplitude of Lys incorporation peak can be attributed to a slower step following peptide bond formation. Similar rates were calculated for tetrapeptides formation using constructs programmed with minus pseudoknot mRNA (+/-) $\left(5.4 \mathrm{~s}^{-1}\right)$ and minus slippery site mRNA (-/+) $\left(4.1 \mathrm{~s}^{-1}\right)($ Table 1$)$. Collectively, our analysis at the extended slippery site (UAU UUA AAG) demonstrates that in all the ribosome complexes programmed with various model mRNAs, the rate for incorporation of the first three amino acids Tyr, Leu and Lys remains unchanged (Table 1), whereas the rate and the proportion of Phe and Val incorporation depend on the elements at the frameshifting site.

A remarkable difference was found in the rate of Phe incorporation at the UUU codon positioned directly $3^{\prime}$ of the slippery heptamer (UUUAAAG) in the zero frame. With frameshifting mRNA (+/+) Phe was incorporated $>80$-fold slower compared to control (-/-) complexes $\left(0.05 \mathrm{~s}^{-1}\right.$ vs $\left.4.1 \mathrm{~s}^{-1}\right)$ or complexes programmed with minus pseudoknot mRNA (+/-) $\left(5.6 \mathrm{~s}^{-1}\right)$ (Figure 10A-C). 
Table 1. Effect of frameshifting elements on peptide synthesis.

\begin{tabular}{cccccc}
\hline & & & & -1 frame & 0 frame \\
Slippery site / Pseudoknot & Tyr, $\mathrm{k}_{1}\left(\mathrm{~s}^{-1}\right)$ & Leu, $\mathrm{k}_{2}\left(\mathrm{~s}^{-1}\right)$ & Lys, $\mathrm{k}_{3}\left(\mathrm{~s}^{-1}\right)$ & Val, $\mathrm{k}_{4}\left(\mathrm{~s}^{-1}\right)$ & Phe, $\mathrm{k}_{5}\left(\mathrm{~s}^{-1}\right)$ \\
\hline + / & $9.1 \pm 0.4$ & $1.7 \pm 0.1$ & $4.6 \pm 0.7$ & $0.3 \pm 0.02$ & $0.05 \pm 0.01$ \\
+ / - & $8.5 \pm 0.3$ & $1.4 \pm 0.1$ & $5.4 \pm 0.8$ & $2.1 \pm 0.5$ & $5.6 \pm 1.2$ \\
- / & $8.0 \pm 0.3$ & $2.9 \pm 0.2$ & $4.1 \pm 0.4$ & $0.09 \pm 0.02$ & $0.06 \pm 0.01$ \\
- / & $8.6 \pm 0.3$ & $2.4 \pm 0.1$ & $4.9 \pm 0.6$ & $0.4 \pm 0.1$ & $4.1 \pm 0.5$ \\
\cline { 2 - 6 } & & & & 0 & 0 frame \\
- / - & $10.3 \pm 0.6$ & $2.2 \pm 0.2$ & $5.2 \pm 0.9$ & N.A. & $4.0 \pm 0.7$ \\
\hline
\end{tabular}

When the ribosomes shift to the -1 reading frame, Val is added to the peptide chain instead of Phe. Formation of the corresponding pentapeptide (fMYLKV) with frameshifting mRNA $(+/+)$ took place at a rate of $0.3 \mathrm{~s}^{-1}$. With control constructs $(-/-)$, Val was incorporated at a rate of $0.4 \mathrm{~s}^{-1}$ (Table 1). Another parameter that can be extracted from the analysis of the peptides would be the relative concentrations of the C-terminal peptide products, which reflects the overall frameshifting efficiency. During the synthesis of the pentapeptides, despite the similar rates for Val incorporation, the amounts incorporated at the C-terminus differed greatly. With frameshifting mRNA $(+/+)$, Val was incorporated in $75 \%$ of the pentapeptide products, whereas Phe was added in 25\% (Figure 10A). With the control construct $(-/-)$, Val was encoded in $9 \%$ of the cases, whereas the remaining $90 \%$ of the control ribosomes incorporated Phe (Figure 10C).

To further explore the effect of individual frameshifting stimulatory elements, decoding was analyzed using mRNA construct without pseudoknot (+/-). About $30 \%$ of the total synthesized pentapeptides contained C-terminal Val. Interestingly, Val was incorporated in the -1 reading frame around seven times faster (apparent rate of $2.1 \mathrm{~s}^{-1}$ ) compared to frameshifting constructs. In order to determine whether this rate was similar to the rate of Val incorporation in the zero frame, we have generated another model mRNA (MetTyrLeuLysVal) displaying GUU codon at the A site, which is encoded as Val. In this construct, the rate of Val incorporation was twofold faster $\left(4.0 \mathrm{~s}^{-1}\right)$ than the construct 
without pseudoknot (+/-) (Figure 10D). This demonstrated that, at the recoding site, Val is incorporated in -1 frame at a rate comparable to that in zero frame. These results are consistent with the observation that -1 frameshifting occurs in the absence of the pseudoknot in vivo (Figure 6B, Figure 10B).

Once we observed that -1 frame decoding was greatly increased in the presence of the slippery site, we were interested to examine the role of the pseudoknot alone in accuracy and speed of translation. For this purpose, an mRNA containing a disrupted slippery site was designed based on the frameshifting mRNA $(+/+)$. When analogous experiments were performed with complexes programmed with mRNA with pseudoknot, without slippery site $(-/+)$, we found that rates for incorporation of Phe and Val were greatly reduced (to $0.06 \mathrm{~s}^{-1}$ and $0.09 \mathrm{~s}^{-1}$, respectively) as compared to control complexes (-/-), whereas the rates for the incorporation of first three amino acids (Tyr, Leu and Lys) were unchanged (Table 1). The efficiency of pentapeptide formation was lower, which would be an indication of pausing or stalling of translation upon encountering the pseudoknot.

Kinetics of amino acid incorpotation for the codon adjacent to the slippery site, decoding in -1 frame as well as in zero frame, were somewhat slower in complexes programmed with frameshifting mRNA (+/+) and mRNA without slippery site (-/+), suggesting that the presence of a pseudoknot slows down the translation over the recoding site. However, it is not clear whether this could be the driving force for the ribosomes to shift the reading frame. Furthermore, at the slippery site U UUA AAG the rates of incorporation of Leu and Lys were similar for all different constructs tested. This suggests that frameshifting takes place during or following the incorporation of Lys to the nascent peptide and a step following peptide bond formation is likely rate-limiting. At the next step, we were prompted to look in more detail at the decoding step of Lys.

\subsubsection{Decoding of lysine at the frameshifting site}

Frameshifting on the XXXA AAG slippery sequence was proposed to be caused by the lack of tRNA $^{\text {Lys }}$ (anticodon UUC) in E. coli (Tsuchihashi and Brown, 1992). In E. coli both AAA and AAG codons are decoded by the same isoacceptor tRNA (anticodon UUU* with modified nucleotide at the first position of the anticodon). The modified nucleotide $\mathrm{mnm}^{5} \mathrm{~s}^{2} \mathrm{U}\left(34 \mathrm{U}^{*}\right)$ 
in $E$. coli tRNA ${ }^{\text {Lys }}$ base pairs with different strengths with $A$ and $G$ nucleotides at the third position of the two Lys codons (Murphy et al., 2004; Yarian et al., 2000; Yokoyama and Nishimura, 1995). In addition it was reported that 3' A containing codons are translated faster than G containing codons in vivo for Glu codons (Krüger et al., 1998). It was proposed that because of the weak codon-anticodon interactions, frameshifting would occur during decoding of the AAG codon (Bertrand et al., 2002; Napthine et al., 2003; Tsuchihashi and Brown, 1992). However, it would be impossible to verify this with peptide analysis, because in both cases the same peptide with C-terminal Lys would be detected. Decoding of AAA is faster than AAG on Lys codons as monitored by the faster rate of peptide bond formation with the AAA compared to AAG codon in the A site (Ortiz-Meoz and Green, 2010). We attempted to test which lysine codon was displayed at the A site in frameshifting $(+/+)$ complexes by measuring the rate of peptide bond formation on AAA and AAG codons.

To monitor accommodation and peptide bond formation on the AAA codon we have generated an mRNA that contains a single $G$ to $A$ point mutation at the lysine codon. In the rest of the model mRNAs used in this study, the AAG codon is present in the zero frame. The experiment was carried out by rapidly reacting initiation complexes programmed with model mRNAs encoding for MYLKV (where lysine codon was either AAA or AAG) with ternary complexes EF-Tu.GTP.aminoacyl-tRNA for Tyr, Leu, Lys and Val in the presence of EF-G. The rates of di- (fMY), tri- (fMYL) peptide formation show no differences among the complexes tested. We note, with the ribosomes programmed with the model mRNAs encoding AAA or AAG on the Lys codon, Lys was incorporated slightly faster (with a rate of $8.6 \mathrm{~s}^{-1}$ ) on the AAA codon, compared the AAG $\left(6.1 \mathrm{~s}^{-1}\right)$ (Table 2). The tendency to decode the AAA codon faster than the AAG codon was in semi-agreement with Ortiz et al. (Ortiz-Meoz and Green, 2010). With the frameshifting construct $(+/+)$ the apparent rate of Lys incorporation was measured around $5 \mathrm{~s}^{-1}$, which is similar to $6.1 \mathrm{~s}^{-1}$ within the statistical significance of the measurements (Table 1). This would suggest that AAG codon was presented at the $A$ site during frameshifting, supporting the notion that tRNA slippage had occurred following decoding of the lysine. 
Table 2. Decoding of lysine codons AAA and AAG codons in a model mRNA without the pseudoknot*.

\begin{tabular}{llr}
\hline & 5' AAA 3' $^{\prime}$ & \multicolumn{1}{c}{ 5' AAG 3' } \\
\hline Tyr, $\mathrm{k}_{1}\left(\mathrm{~s}^{-1}\right)$ & $8.2 \pm 0.6$ & $11.2 \pm 0.6$ \\
Leu, $\mathrm{k}_{2}\left(\mathrm{~s}^{-1}\right)$ & $3.4 \pm 0.4$ & $3.1 \pm 0.2$ \\
Lys, $\mathrm{k}_{3}\left(\mathrm{~s}^{-1}\right)$ & $8.6 \pm 2.1$ & $6.1 \pm 0.8$ \\
\hline
\end{tabular}

* The mRNAs utilized encode for MetTyrLeuLysVal.

\subsubsection{Testing the effect of tRNAs decoding -1 and 0 frames following frameshifting}

Having observed that the amino acid incorporation at the codon directly following the slippery site was slower in case of frameshifting, we tested whether the concentrations of tRNAs that are incorporated after tRNA ${ }^{\text {Lys }}$ are important. If peptidyl-tRNA (MYLK-tRNA ${ }^{\text {Lys }}$ ) would be repositioned on the mRNA during the recognition of the following codon (for Phe or Val) - but before the peptidyl transferase reaction is completed, the efficiency of decoding should depend on the concentration of the potential $A$ site substrates corresponding to codons in -1 frame or zero frame (Barak et al., 1996; Baranov et al., 2004; Kolor et al., 1993). In order to determine whether frameshifting occurred during codon recognition of the next codon UUG, following the Lys codon, we have focused especially on the incorporation of Val and Phe.

First, we investigated whether there was competition between zero and - 1 frame tRNAs for binding to the A site during frameshifting by omitting the potentially competing of the A-site substrates Phe-tRNA ${ }^{\text {Phe }}$ and Val-tRNA ${ }^{\text {Val }}$. In the scenario where frameshifting was caused by hungry codons at the A site, omitting the ternary complex EF-Tu.GTP.Val-tRNA ${ }^{\text {Val }}$ for binding in -1 frame would abolish frameshifting (Barak et al., 1996). We have tested this in the frameshifting construct $(+/+)$ by excluding the -1 frame A-site substrate EF-Tu.GTP.ValtRNA ${ }^{\mathrm{Val}}$. The initiation complexes were reacted in a quenched flow with the ternary complex mix EF-Tu.GTP.aminoacyl-tRNAs (Tyr, Leu, Lys and Phe) in the presence of EF-G. The rate of fMYLKF pentapeptide formation was not increased in the absence of Val-tRNA ${ }^{\text {Val }}\left(k=0.01 \mathrm{~s}^{-1}\right)$ (Figure 11), which was not significantly different from the rate $\left(0.05 \mathrm{~s}^{-1}\right)$ obtained with both 
tRNAs (Val and Phe) present (Table 1). This showed that the efficiency of zero frame decoding was not increased and implied that frameshifting is not caused by competition between the tRNAs reading the codons after the slippery site.

In case limited availability of cognate tRNA would facilitate binding of aminoacyl tRNAs -1 frame, omitting the zero frame substrate EF-Tu.GTP.Phe-tRNA ${ }^{\text {Phe }}$ would lead to increased -1 frameshifting. That was tested by rapidly reacting the initiation complexes programmed with the frameshifting mRNA (+/+) with ternary complexes EF-Tu.GTP-aminoacyl-tRNA (Tyr, Leu, Lys and Val) in the presence of EF-G. The apparent rate of pentapeptide (fMYLKV) formation was $0.2 \mathrm{~s}^{-1}$. This rate was similar to the rate observed for Val incorporation $\left(0.3 \mathrm{~s}^{-1}\right)$ in frameshifting $(+/+)$ constructs in the presence of Phe-tRNA ${ }^{\text {Phe }}$ (Figure $\left.11 \mathrm{~A}\right)$. This showed that the rate of Val incorporation was not different in the absence of zero frame competitor, EFTu.GTP.Phe-tRNA ${ }^{\text {Phe }}$, demonstrating that the efficiency of frameshifting is not influenced by tRNA competition for mRNA decoding after the slippery site.

A Without -1 frame competitor tRNA

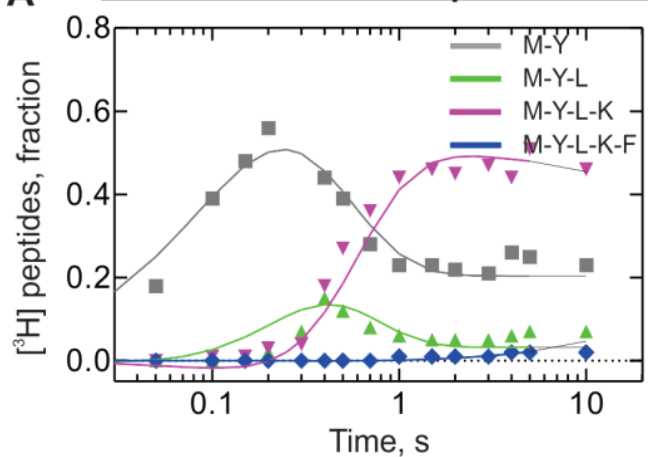

B Without zero-frame competitor tRNA

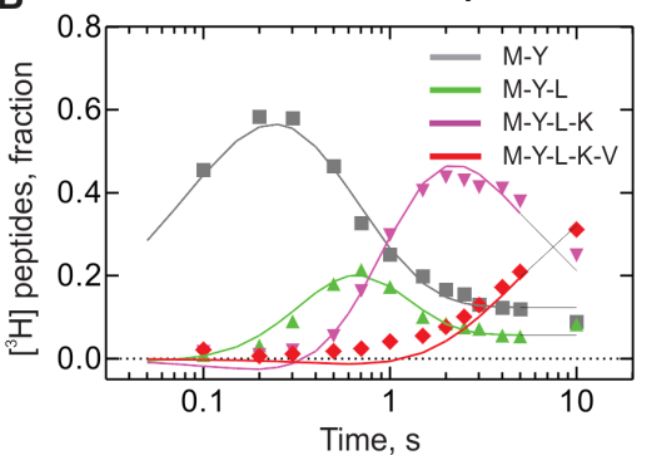

Figure 11. Competition between EF-Tu.GTP.Phe-tRNA ${ }^{\text {Phe }}$ and EF-Tu.GTP.Val-tRNA ${ }^{\text {Val }}$ for binding to the A site. 70S initiation complexes programmed with frameshifting mRNA $(+/+)(0.2 \mu \mathrm{M})$ were rapidly reacted with ternary complex mix composed of EF-Tu.GTP-aminoacyl-tRNA for Tyr, Leu, Lys, Phe (A), or Tyr, Leu, Lys, Val (B) in the presence of EF-G $(2 \mu \mathrm{M})$.

As a second approach, we tested whether the concentration of the A site substrates that potentially bind at the -1 or zero frames would lead to increased decoding in -1 frame. Therefore, we varied the concentrations of either zero or -1 frame aminoacyl-tRNA substrates (Phe-tRNA ${ }^{\text {Phe }}$ and Val-tRNA ${ }^{\text {Val }}$, respectively). When - 1 frame substrate Val-tRNA ${ }^{\text {Val }}$ was used in excess, rate of Val incorporation was $0.3 \mathrm{~s}^{-1}$. Zero-frame decoding was not effected as shown by the rate of Phe incorporation at the pentapeptides (fMYLKF) of $0.05 \mathrm{~s}^{-1}$ 
(compare to Table 1). In case Phe-tRNA ${ }^{\text {Phe }}$ was introduced in excess to the A site, the rate of Phe incorporation was essentially not changed. In this case as well, no change in the kinetics of -1 frame decoding of Val was observed $\left(0.4 \mathrm{~s}^{-1}\right)$ (Table 3$)$. These results showed that the observed rate limiting step of the pentapeptide formation precedes the decoding step of the next codon, UUU.

Table 3. Competition at the recoding site. The rates of pentapeptide formation were monitored at varying concentrations of Val and Phe ternary complexes as A-site substrates on the 3' G UUU 5' stretch.

\begin{tabular}{llrrrrc}
\hline \multicolumn{2}{l}{ TC concentration } & & & & -1 frame & 0 frame \\
\cline { 1 - 1 }$[$ Val], $\mu \mathrm{M}$ & {$[$ Phe $], \mu \mathrm{M}$} & Tyr, $\mathrm{k}_{1}\left(\mathrm{~s}^{-1}\right)$ & Leu, $\mathrm{k}_{2}\left(\mathrm{~s}^{-1}\right)$ & Lys, $\mathrm{k}_{3}\left(\mathrm{~s}^{-1}\right)$ & Val, $\mathrm{k}_{4}\left(\mathrm{~s}^{-1}\right)$ & Phe, $\mathrm{k}_{5}\left(\mathrm{~s}^{-1}\right)$ \\
\hline 1.5 & 1.5 & $9.1 \pm 0.4$ & $1.7 \pm 0.1$ & $4.6 \pm 0.7$ & $0.3 \pm 0.02$ & $0.05 \pm 0.01$ \\
0 & 1.5 & $7.9 \pm 0.6$ & $2.9 \pm 0.4$ & $6.9 \pm 1.6$ & N.A & $0.01 \pm 0.05$ \\
1.5 & 0 & $9.2 \pm 0.5$ & $1.6 \pm 0.1$ & $3.2 \pm 0.5$ & $0.2 \pm 0.05$ & N.A \\
1 & 4 & $9.9 \pm 0.6$ & $1.9 \pm 0.1$ & $3.2 \pm 0.5$ & $0.5 \pm 0.06$ & $0.06 \pm 0.02$ \\
4 & 1.5 & $10.5 \pm 0.9$ & $2.1 \pm 0.2$ & $4.6 \pm 1.1$ & $0.3 \pm 0.05$ & $0.05 \pm 0.01$ \\
\hline
\end{tabular}

\subsection{Time-resolved puromycin reactivity and $50 \mathrm{~S}$ translocation at the frameshifting codon}

Our kinetic studies described above suggested that frameshifting is not an A site-dependent event, i.e. that translating ribosomes shift to the new reading frame after incorporation of Lys, but before the incorporation of Phe or Val, which leaves the translocation of tRNA ${ }^{\text {Lys }}$ as the most likely step at which frameshifting takes place. To test which elemental step of translocation is affected, we measured the reactivity of post-translocation state ribosomes with puromycin which can be used to measure translocation on the 50S subunit (Borowski et al., 1996; Katunin et al., 2002; Semenkov et al., 1992; Wohlgemuth et al., 2008). We attempted to combine our in vitro translation system with the puromycin assay (Katunin et al., 2002) for which we have designed a double mixing experiment in the quench flow. In the first mixing step, 70S initiation complexes are reacted with ternary complexes and EF-G as described above and incubated in one of the reaction loops. In the second step, the complexes were mixed with the A-site aminoacyl-tRNA analog puromycin used in the 
quencher syringe; and the reactants were hold in the exit line allowing completion of the puromycin reaction. After appearing from the exit line the reactions were quenched with $\mathrm{KOH}$ in the collection tube. Setting up of this assay required several optimizations to be performed (i) to define the puromycin reaction time in the exit line, and (ii) validate the method by comparing the rate constants of puromycin reaction with unpurified POST Phe complexes with $\mathrm{fMetPhe-tRNA}^{\text {Phe }}$ at the P site obtained with the present setup and with the conventional mixing (Katunin et al., 2002).

To determine the time required to pass through the exit line, we performed an experiment where we measured puromycin reactivity of the P-site fMet-tRNA ${ }^{\text {MMet }}$. Initiation complexes 70S-mRNA-fMet-tRNA ${ }^{\text {fMet }}$ were rapidly mixed with puromycin in the reaction loop and after the given delay time (in this case reaction time with puromycin) pushed into a collection tube containing the quencher to stop the puromycin reaction. The analysis of the reaction products showed that about $80 \%$ of the P-site $\mathrm{fMet}^{-\mathrm{tRNA}}{ }^{\mathrm{fMet}}$ reacts with Pmn after $2 \mathrm{~s}$ (Figure 12). We evaluated the curves by single exponential fitting and determined the apparent rate of the Pmn reaction with the initiation complexes as $1 \mathrm{~s}^{-1}$. The time spent in the exit line before the puromycin reaction could be stopped was estimated as 0.4-0.5 s from the $X$ intercept - which was taken into account in the next experiments, where POST complexes were reacted with Pmn.

A

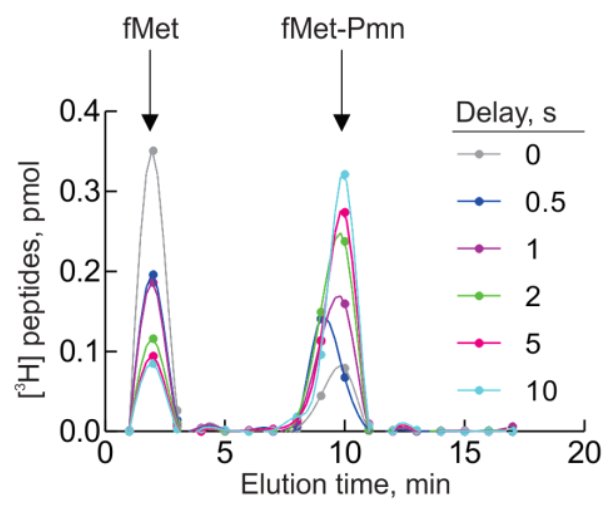

B

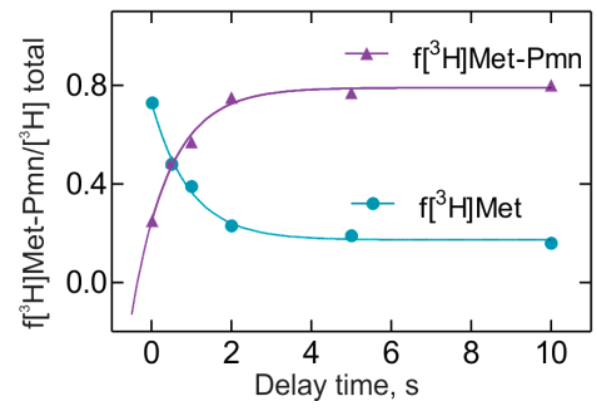

Figure 12. fMet-puromycin reactivity monitored by quench flow. (A) Separation of puromycin reaction products is shown. (B) Fraction of puromycin reactive 705 initiation complexes ( $f\left[{ }^{3} \mathrm{H}\right]$ Met-Pmn/ $\left[{ }^{3} \mathrm{H}\right]$ total) at each time point was plotted. Kinetics of formation of the $\left.\mathrm{f}^{3} \mathrm{H}\right]$ MetPmn peptide was determined by single exponential fitting $\left(k=1 \mathrm{~s}^{-1}\right)$. 
A classical pretranslocation complex with the P-site occupied with initiator tRNA ${ }^{\text {fMet }}$ and the A-site with fMetPhe-tRNA $^{\text {Phe }}$ is essentially nonreactive with puromycin, whereas posttranslocation complexes containing peptidyl-tRNA in the P/P state react more than a 1000 times faster (Peske et al., 2004; Semenkov et al., 1992; Sharma et al., 2004). In the next step, we measured the rate of puromycin reaction of a single-round translocated complex with peptidyl-tRNA ${ }^{\text {Phe }}$, which was a test for the double mixing experiment in the quench flow.

The experiment was carried out in two subsequent steps in the quench flow. In the first step, initiation complexes with the P-site occupied by fMet-tRNA ${ }^{f M e t}$ were rapidly reacted with EFTu.GTP.Phe-tRNA ${ }^{\text {Phe }}$ in the presence of EF-G for a fixed time $(0.5 \mathrm{~s})$ that allow formation of peptidyl tRNA ${ }^{\text {Phe }}$. In the reaction loop (depicted as 1 in Figure $13 A$ ) the A-site binding of PhetRNA $^{\text {Phe }}$ would be followed by translocation of fMetPhe-tRNA ${ }^{\text {Phe }}$ to the P site rendering the $3^{\prime}$ of the tRNA reactive for puromycin. In the second step, the complexes were reacted with puromycin - instead of quencher - (Figure 13A). The time of puromycin reaction varied by introducing delays in the second mixing step before the reactions were stopped by $\mathrm{KOH}$ in a collection tube. Note that the $0.4-0.5 \mathrm{~s}$ in the exit line (depicted as 3 in Figure 13A) had to be added to the puromycin reaction time. By that, we monitored the reaction of $\mathrm{P}$-site bound fMetPhe-tRNA $^{\text {Phe }}$ with puromycin, which resulted in formation of a tripeptide (fMetPhePmn). $\left[{ }^{3} \mathrm{H}\right]$-labeled peptides corresponding to puromycin-reactive and non-reactive fractions over the total $\left[{ }^{3} \mathrm{H}\right]$-labeled peptides were quantified. Exponential fitting gave a rate of the puromycin reaction of $2.0 \mathrm{~s}^{-1}$ (Figure 13B). A similar rate was obtained from purified POST Phe $_{\text {P }}$ complexes at $1 \mathrm{mM}$ puromycin concentration (Katunin et al., 2002), which indicates that the mixing scheme used in the present experiments yields the same rate constants for puromycin reaction as the conventional mixing. 
A

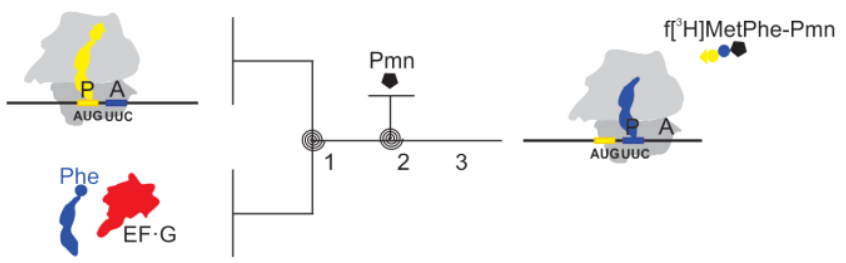

B

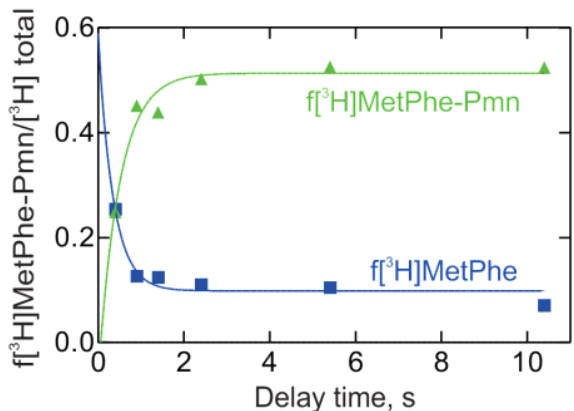

Figure 13. Puromycin reactivity of $f\left[{ }^{3} \mathrm{H}\right]$ MetPhe-tRNA ${ }^{\text {Phe }}$. (A) Schematic of the experiment. (Step 1) Initiation complexes, 70S. $\left[^{3} \mathrm{H}\right] \mathrm{Met}^{\mathrm{T}} \mathrm{tRNA}{ }^{\mathrm{fMet}} \cdot \mathrm{mRNA}(0.1 \mu \mathrm{M})$ were rapidly mixed with ternary complex, EF-Tu.GTP.Phe-tRNA ${ }^{\text {Phe }}(1 \mu \mathrm{M})$ in the presence of EF-G $(2 \mu \mathrm{M})$ for $0.5 \mathrm{~s}$ in the reaction loop (depicted as 1 ). (Step 2) The complexes with $\mathrm{FMetPhe-tRNA}^{\text {Phe }}$ in the P-site were mixed with puromycin (final $1 \mathrm{mM}$ ) (depicted as 2) and reacted for indicated time intervals in the exit line (depicted as 3 ) before the reactions were quenched with $\mathrm{KOH}(0.4 \mathrm{M})$ in the collection tube. (B) Time courses were evaluated by exponential fitting (continuous lines). $\left[{ }^{3} \mathrm{H}\right] \mathrm{fMetPhe}$, as a substrate, was consumed at a rate of $2.8 \mathrm{~s}^{-1}$ (in blue, squares) and $\left[{ }^{3} \mathrm{H}\right] \mathrm{fMetPhe}-\mathrm{Pmn}$ formed at a rate of $2.0 \mathrm{~s}^{-1}$ (in green, triangles).

These experiments allowed us to establish the system for monitoring puromycin reaction in real-time during translocation using non-purified POST complexes. To measure translocation of $\mathrm{fMYLK} \mathrm{tRNA} \mathrm{L}^{\text {Lys }}$, purified $70 \mathrm{~S}$ initiation complexes programmed with defined model frameshifting $(+/+)$, control (-/-) and minus pseudoknot (+/-) mRNAs (70S.fMettRNA $^{\text {fMet }} \cdot \mathrm{mRNA}$ ) were rapidly mixed with ternary complexes EF-Tu.GTP-aminoacyl-tRNAs for Tyr, Leu and Lys in presence of EF-G to form fMYLK-tRNA ${ }^{\text {Lys }}$, which at the end of the reaction should be located in the $\mathrm{P}$ site, where it can further react with puromycin. After the indicated time points in the reaction loops (depicted as 1 in Figure 14A), complexes were mixed with puromycin and were pushed through the exit line (depicted as 3 ), where they reacted with puromycin for $\sim 1 \mathrm{~s}$ (Figure 14A). Concerning the analysis of the peptides in principle, di- (fMY) and tri- ( $f M Y L)$ peptides could react with puromycin to a certain extent, which could make the analysis of this experiment complicated. However, as only C-terminal Lys and Lys-Pmn would contain the $\left[{ }^{14} \mathrm{C}\right]$-Lys label, we could analyze the fMYLK and fMYLKPmn peptides independently of reactions of the shorter peptides. The chromatographic elution profile of the puromycin reactive tetrapeptides (fMYLK-Pmn) had a characteristic shift of 10 min with respect to the tetrapeptides (fMYLK). The fraction of $f\left[{ }^{3} \mathrm{H}\right] \mathrm{MYL}-\left[{ }^{14} \mathrm{C}\right] \mathrm{K}-$ Pmn over the total $\left[{ }^{3} \mathrm{H}\right]$ peptides (normalized) were plotted as a function of time, and the resulting curves were fitted by single exponential functions. 
A

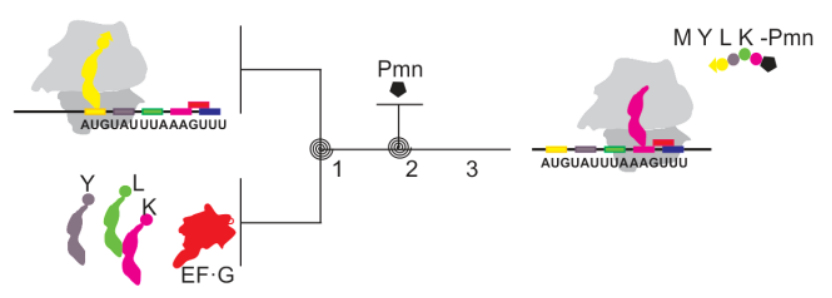

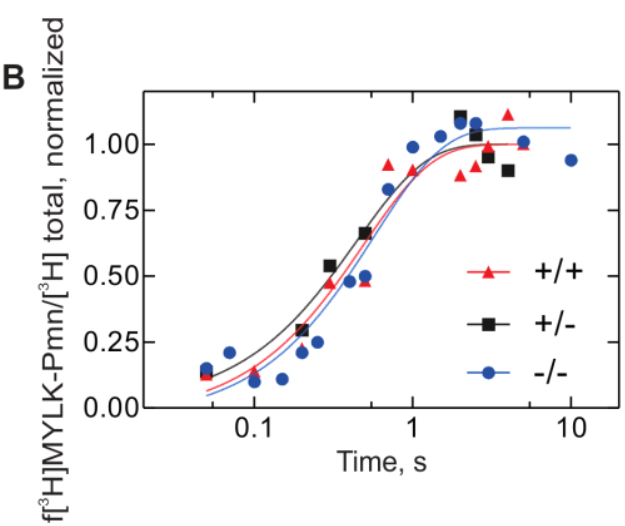

Figure 14. Time-resolved $50 \mathrm{~S}$ translocation of $\mathrm{fMYLK}-\mathrm{tRNA} \mathrm{A}^{\text {Lys }}$ complexes measured by indicator puromycin reaction. (A) The reactants were rapidly mixed in a double mixing experiment in quench flow. (Step 1) Initiation complexes 70S.fMet-tRNA ${ }^{\text {fMet }} \cdot \mathrm{mRNA},(0.2 \mu \mathrm{M})$ were rapidly mixed with ternary complexes, EF-Tu.GTP.aminoacyl-tRNA for Tyr, Leu and Lys (1.5 $\mu \mathrm{M})$ and EF-G $(2 \mu \mathrm{M})$ in reaction loops (depicted as 1$)$ for the indicated times. (Step 2) Puromycin (1 $\mathrm{mM}$ ) was mixed with the complexes (depicted as 2). After passing the exit line (depicted in 3 ) the reaction was quenched with $\mathrm{KOH}$ in a collection tube. (B) The fraction of $\mathrm{f}\left[{ }^{3} \mathrm{H}\right] \mathrm{MYL}-\left[{ }^{14} \mathrm{C}\right] \mathrm{K}$ Pmn over total $\left[{ }^{3} \mathrm{H}\right]$ peptides (normalized to 1 ) was plotted at different reaction times and evaluated by single exponential fitting. Calculated rates of puromycin reaction for complexes programmed with frameshifting mRNA $(+/+)$, depicted in red, $\mathrm{k}\left(\mathrm{s}^{-1}\right)=2.1 \pm 0.3$, complexes programmed with mRNA with slippery site and minus pseudoknot, depicted in black $(+/-), \mathrm{k}\left(\mathrm{s}^{-1}\right)$ $=2.2 \pm 0.5$ and complexes programmed with mRNA minus slippery site and minus pseudoknot, depicted in blue $\left.(-/-), \mathrm{k}^{-1}\right)=1.7 \pm 0.3$.

The rate of puromycin reaction of $\mathrm{FMYLK}$-tRNA ${ }^{\text {Lys }}$ synthesized on the frameshifting mRNA $(+/+)$ was $2.1 \pm 0.3 \mathrm{~s}^{-1}$. Notably, because puromycin reaction of the tRNA carrying the Cterminal Lys residue is intrinsically very high $\left(100 \mathrm{~s}^{-1}\right)$ (Wohlgemuth et al., 2008), the rate measured here reflects the rate of translocation of $\mathrm{FMYLK}$-tRNA ${ }^{\text {Lys }}$ from the $A$ to the $P$ site on the $50 \mathrm{~S}$ subunit. Similarly, in the control construct (-/-) fMYLK-tRNA ${ }^{\text {Lys }}$ reacted with puromycin at a rate of $1.7 \pm 0.3 \mathrm{~s}^{-1}$. Furthermore, ribosome complexes programmed with mRNA that was mutated for the pseudoknot (+/-) had rate of $2.2 \pm 0.5 \mathrm{~s}^{-1}$ (Figure 14B). Collectively, translocation as measured by puromycin reactivity of different ribosomal complexes with or without frameshifting elements indicated that the -1 frameshifting occurs after the peptide bond formation and is not dependent on $50 \mathrm{~S}$ translocation.

\subsection{Movement of frameshifting tRNA ${ }^{\text {Lys }}$ from the $A$ to the $P$ site}

Transient changes on the tRNA molecule during accommodation or translocation can be followed by proflavin attached at the D loop of tRNA (Rodnina et al., 1997; Rodnina et al., 
1994; Semenkov et al., 2000; Wilden et al., 2006). In order to monitor the movement of tRNA $^{\text {Lys }}$ from the $A$ site to the $P$ site on the 50 S subunit with frameshifting and control complexes, we performed stopped-flow experiments using proflavin-labeled Lys-tRNA ${ }^{\text {Lys }}$. Translation of the codon sequence (MetTyrLeuLysPhe) was initiated by rapidly mixing $70 \mathrm{~S}$ initiation complexes programmed with frameshifting $(+/+)$ and control $(-/-)$ mRNAs with ternary complexes EF-Tu.GTP-aminoacyl-tRNA for Tyr, Leu, Lys (Prf), Val and Phe in the presence of EF-G and the fluorescence change of tRNA ${ }^{\text {Lys }}$ (Prf) was monitored, which allowed us to selectively monitor the binding and translocation of tRNA ${ }^{\text {Lys }}$ during translation (Figure $15 A)$.

A

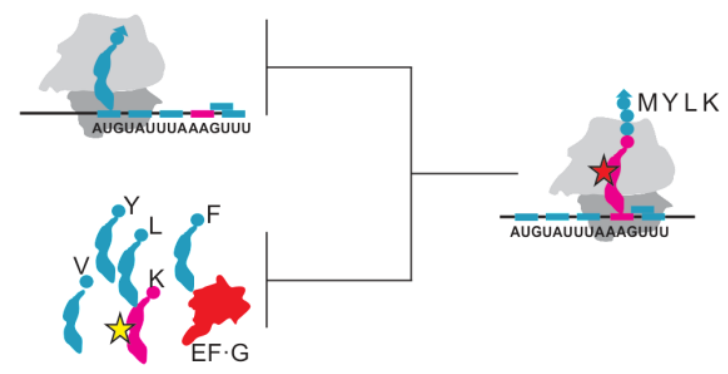

B

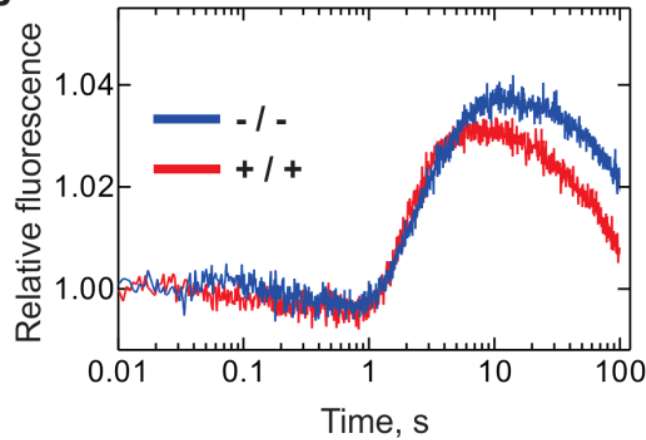

Figure 15. Translocation of $t R N A^{\text {Lys }}$ at the $50 \mathrm{~S}$ subunit. (A) Experimental setup. Initiation complexes $(0.2 \mu \mathrm{M})$ programmed with frameshifting $(+/+)$ or control $(-/-)$ mRNAs were rapidly reacted with ternary complexes EF-Tu.GTP.aminoacyl-tRNAs for Tyr, Leu, Lys (Prf), Val and Phe (1.5 $\mu \mathrm{M}$ each) in the presence of EF-G $(2 \mu \mathrm{M})$ in the stopped-flow apparatus. (B) Time course of fluorescence change of $\operatorname{tRNA}^{\text {Lys }}$ (Prf) on a frameshifting $(+/+$, red) or control $(-/-$, blue) complexes.

The fluorescence of tRNA ${ }^{\text {Lys }}$ (Prf) increased after a delay of about $1 \mathrm{~s}$, which probably reflected all elongation steps preceding translocation of tRNA ${ }^{\text {Lys }}$. After that delay, a large biphasic fluorescence change was observed. The first phase most likely reflects translocation upon displacement of $\operatorname{tRNA}^{\mathrm{Lys}}$ (Prf) from the A site to the P site, where the fluorescence signal increased, similarly to the effect reported for the translocation of fMetPhe-tRNA ${ }^{\text {Phe }}$ (Rodnina et al., 1997; Savelsbergh et al., 2003). The second phase comprised a very slow decrease in fluorescence, which could be attributed to the following events such as dissociation of tRNA ${ }^{\text {Lys }}$ from the ribosome. The rates obtained by three exponential fitting yielded an apparent rate of $0.5 \mathrm{~s}^{-1}$ for the translocation of $\mathrm{tRNA} \mathrm{A}^{\mathrm{Lys}}$ on ribosomes programmed with control mRNA lacking frameshifting elements (-/-). With complexes programmed with 
frameshifting mRNA $(+/+)$ the rate of tRNA ${ }^{\text {Lys }}$ translocation was essentially not changed $(0.9$ $\mathrm{s}^{-1}$ ) (Figure 15B). Thus, the overall rate of translocation as followed by a label at the elbow region of the tRNA is not affected by frameshifting.

To conclude, the extent and rates of translocation was the same on the frameshifting $(+/+)$ and minus slippery site minus pseudoknot (-/-) complexes during translocation of the tRNA $^{\text {Lys }}$ (Prf) at the $50 \mathrm{~S}$ subunit. This further supports the results of the $50 \mathrm{~S}$ translocation assay as measured by puromycin reactivity and leaves the incomplete (frameshifting) translocation on the $30 \mathrm{~S}$ subunit as the only remaining step between the translocation of tRNA $^{\text {Lys }}$ and decoding of the codons in the zero or -1 frame by tRNA ${ }^{\text {Phe }}$ or tRNA ${ }^{\text {Val }}$.

\subsection{EF-G binding and $30 \mathrm{~S}$ translocation}

To explore why translocation on the $30 \mathrm{~S}$ subunit is affected, we monitored the interaction of EF-G with the ribosome using FRET between labels in the $L 7 / 12$ stalk of the ribosome and in EF-G.

The L7/L12 protein has been shown to interact with EF-G and EF-Tu which has structural and functional implications (Diaconu et al., 2005; Kothe et al., 2004; Mohr et al., 2002; Savelsbergh et al., 2000; Stark et al., 2000). Recently, a novel fluorescence assay was developed in our laboratory utilizing FRET between a fluorescent dye (Alx488) covalently attached to the protein L7/12 and a quencher dye (QSY9) attached at position 209 in domain 1 of EF-G. The fluorescence of the L12 protein was quenched upon EF-G interaction (C. E. Cunha, A. Lehwess-Litzmann, personal communication). The assay was modified in order to monitor EF-G (QSY9) binding when the ribosome translates over the frameshifting codon.

Complexes were prepared with the L7/12-labeled ribosomes that contained $\mathrm{FMYL-tRNA}{ }^{\text {Leu }}$ in

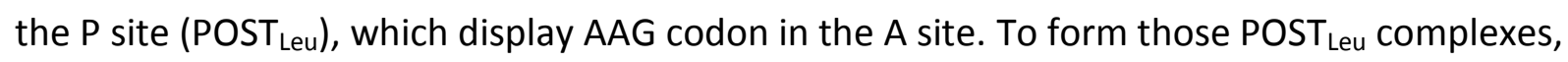
initiated $70 \mathrm{~S}$ ribosomes labeled at L7/12 with Alx488 were incubated with ternary complexes, EF-Tu.GTP.aminoacyl-tRNAs for Tyr and Leu in the presence of EF-G to allow the translocation of $\mathrm{fMYL-tRNA}{ }^{\text {Leu }}$ to the $P$ site. Ternary complexes and initiation complexes were used without further purification. Afterwards, the POST Leu complexes were rapidly reacted in a stopped flow with EF-Tu.GTP.Lys-tRNA ${ }^{\text {Lys }}$ and EF-G (QSY9), by which Lys-tRNA ${ }^{\text {Lys }}$ will initially 
bind to the $A$ site and form pretranslocation complex with fMYLK-tRNA ${ }^{\text {Lys }}$ in the A site. This would be followed by EF-G (QSY9) binding and translocation (Figure 16A).

A

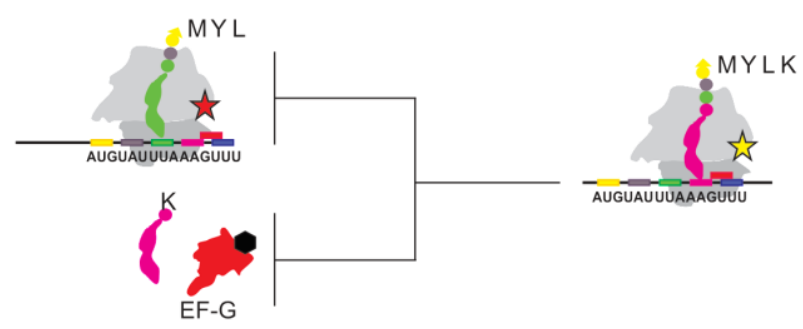

C

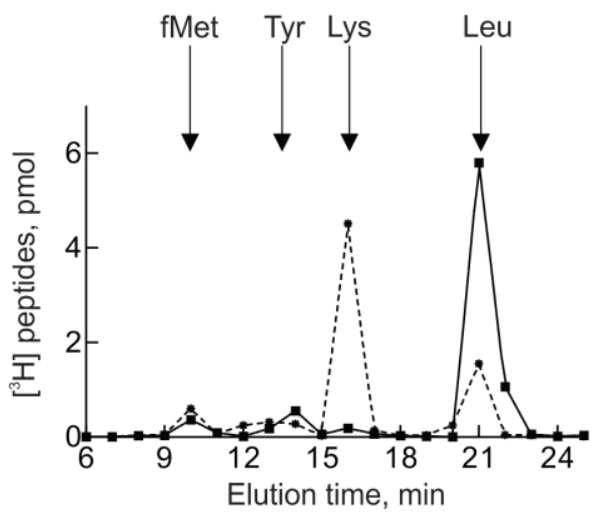

B

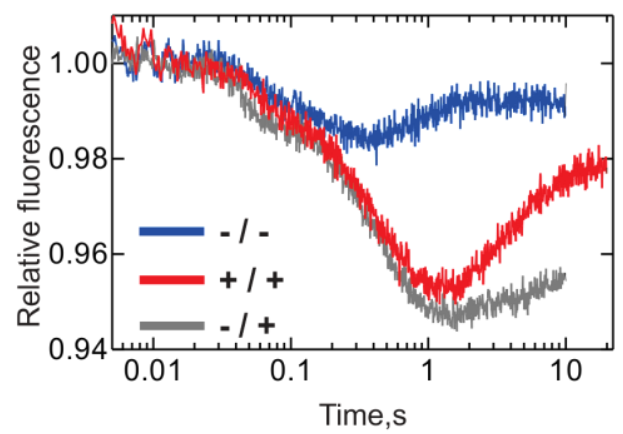

Figure 16. EF-G binding to ribosomes translating the Lys codon. (A) Schematic representation

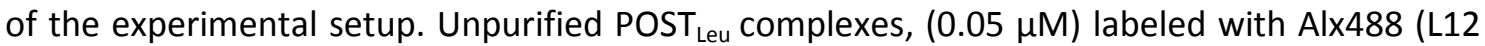
position 138) were rapidly mixed with EF-Tu.GTP.Lys-tRNA ${ }^{\text {Lys }}(0.25 \mu \mathrm{M})$ and EF-G (209 QSY9) (1 $\mu \mathrm{M})$ in a stopped flow apparatus. (B) Fluorescence traces of individual POST $_{\text {Lys }}$ complexes programmed with frameshifting (+/+) (in red), control (-/-) (in blue) and minus slippery site (/+) (in grey) mRNAs. (C) Peptide products of POST Leu complexes before (solid line) and after addition of EF-Tu.GTP.Lys-tRNA ${ }^{\text {Lys }}$ and EF-G (QSY9) (dotted line).

When EF-G binding to POST $_{\text {Leu }}$ complexes programmed with control mRNA (-/-) was monitored, a decrease in fluorescence $\left(k_{\text {app }}\right.$ of $\left.6 \mathrm{~s}^{-1}\right)$ was followed by an increase in fluorescence $\left(2.1 \mathrm{~s}^{-1}\right)$. When an analogous experiment was conducted with ribosome complexes programmed with frameshifting mRNA (+/+), a decrease in fluorescent signal (1.9 $\mathrm{s}^{-1}$ ) was followed by a slower increase in fluorescence $\left(0.5 \mathrm{~s}^{-1}\right)$. Initial quenching of fluorescence was remarkably larger in frameshifting $(+/+)$ complexes, which reported on binding of EF-G.GTP to ribosomal complexes during Lys translocation (Figure 16B).

Next, we tested whether presence of the pseudoknot could influence translocation and the time that EF-G interacts with the ribosomes. For this purpose, EF-G binding on POST Leu 
complexes programmed with mRNA without the slippery site $(-/+)$ was examined. We observed a similar biphasic fluorescence quenching as in frameshifting (+/+) complexes, with the major step proceeding with a rate of $1.9 \mathrm{~s}^{-1}$. The following fluorescence increase $\left(0.6 \mathrm{~s}^{-1}\right)$ had a smaller amplitude change compared to frameshifting $(+/+)$ or to the control (-/-) complexes. This might report that in the POST $_{\text {Leu }}$ complexes programmed with the mRNA without the slippery site $(-/+)$ dissociation of EF-G was slowed down. These results claim that EF-G remains bound on the ribosomes longer during frameshifting, as well as in the presence of pseudoknot.

To verify the biochemical properties of the complexes, we analyzed the formation of the tetrapeptide (fMYLK) from the $\mathrm{POST}_{\text {Leu }}$ complexes (Figure 16C). Purified initiated $70 \mathrm{~S}$ ribosomes were incubated with ternary complexes for Tyr and Leu in the presence of EF-G to allow the translocation of tRNA ${ }^{\text {Leu }}$ in the $\mathrm{P}$ site and the reaction product was analyzed by HPLC. Then, EF-Tu.GTP. $\left[{ }^{14} \mathrm{C}\right]$ Lys-tRNA ${ }^{\text {Lys }}$ and EF-G were added to the POST Leu $_{\text {complexes and }}$ the respective product was analyzed. Overall, $80 \%$ of the POST $_{\text {Leu }}$ complexes reacted with Lys-tRNA ${ }^{\text {Lys }}$, showing that the POST Leu complexes were almost fully active in translation (Figure 16C).

In order to determine whether the effect of EF-G retention on the ribosome during frameshifting was specific to the Lys codon, we carried out similar experiments for the previous codon, Leu. In this case, no significant difference was observed in the fluorescent signal for EF-G binding and the following events (data not shown). In addition, to exclude the possibility that the observed change in fluorescence was due to the binding of EF-G alone or ternary complexes alone, we measured the binding of EF-G to the post translocation tRNA ${ }^{\text {Leu }}$ complexes. No significant change in fluorescence was observed (data not shown). 


\section{DISCUSSION}

Frameshifting has important functions in gene expression especially in viral particle assembly and propagation. For this reason, it has become an attractive target for antiviral drugs. However, it is not known with certainty at which point during translation elongation cycle frameshifting occurs, during or after tRNA accommodation or during translocation. This study shows for the first time the kinetics of different steps of translation elongation during -1 frameshifting and thus provides a new insight into the mechanism of -1 frameshifting. We show that -1 frameshifting occurs during EF-G-catalyzed translocation of tRNAs and mRNA on the 305 subunit and results from the synergetic action of the frameshifting stimulatory elements.

\subsection{Functionality of modified IBV $1 a / 1 b$ frameshifting site in $E$. coli}

In programmed -1 frameshifting, the ribosome is induced to move to an alternative open reading frame. Efficient frameshifting relies on the coordinated function of stimulatory signals embedded in the mRNA. One is the obligatory frameshifting slippery site, which is in most cases a heptanucleotide motif in the form of two homopolymeric triplets (X-XXY-YYZ). The other element is the cis-acting stimulatory RNA found at an optimal distance of 5-8 nucleotides downstream of the slippery site, which is proposed to perturb normal decoding or translocation beyond a pause (Namy et al., 2006; Plant and Dinman, 2005). Lastly, a SD element which is capable of base pairing with the $3^{\prime}$ end of the 16S rRNA is implicated as a stimulatory element in E. coli (Larsen et al., 1994; Mejlhede et al., 1999). Frameshifting efficiencies can be more than $80 \%$ depending on the system utilized (Donly et al., 1990; Tsuchihashi and Brown, 1992).

Frameshifting segments from viral genes have been used to study recoding in bacterial systems (Brierley et al., 1997; Di et al., 1993; Horsfield et al., 1995; Weiss et al., 1989; Yelverton et al., 1994). We utilized a modified form of the IBV coronavirus $1 a / 1 b$ gene fragment as a model for -1 frameshifting. Initially, we established an in vivo dual luciferase assay to test the functionality of this model in E. coli. 
The efficiency of -1 frameshifting in the IBV $1 a / 1 b$ fragment observed in our experiments is up to $70 \%$ in E. coli. In earlier mutational studies, the original IBV $1 a / 1 b$ gene caused $30 \%-1$ frameshifting in vivo in oocyte embryos, or in vitro in rabbit reticulocyte lysate (Brierley et al., 1989). Using a minimal variant of the IBV pseudoknot with a shorter loop 2, which is also utilized in this work, Napthine et al. reported an enhanced frameshifting efficiency of $\sim 50 \%$ in the in vitro translation system in rabbit reticulocyte lysate (Napthine et al., 1999). The response of ribosomes to viral frameshifting elements changes between eukaryotic and prokaryotic translation systems (Brierley et al., 1997; Weiss et al., 1989). In the original IBV $1 a / 1 b$ mRNA the second codon of the slippery site (AAC) coding for Asp was found to be a weak frameshift codon in E. coli, whereas replacement of this codon with AAG (encoding Lys) restored the efficiencies (up to 40\%); the situation was completely the reverse in rabbit reticulocyte lysate. Because the first triplet requirements are less stringent in rabbit reticulocyte lysate, a mismatched G:A (codon:anticodon) pair in the first position can be tolerated, but still certain mutations (like $\mathrm{U}$ to $\mathrm{C}$ of the first position) can dramatically reduce the frameshifting efficiency (down to $2 \%$ ), proposing both codons are involved in tRNA slippage (Brierley et al., 1992). In contrast, mutational work in E. coli suggested that the first codon of the slippery site (UUA) was not involved in tRNA slippage, because the $U$ to $C$ mutation in second position was fully functional, arguing for a single-tRNA slippage mechanism in E. coli (Brierley et al., 1997; Napthine et al., 2003).

Changes of the pseudoknot sequence and structure lead to varying frameshifting efficiencies. In previous studies, wild type or mutant variants of the IBV $1 a / 1 b$ pseudoknots were tested for their efficiencies in promoting -1 frameshifting. Using the in vitro rabbit reticulocyte translation system, Brierley et al. reported complete abolishment of frameshifting in the absence of the pseudoknot (Brierley et al., 1991). In vivo in E. coli deletion of the pseudoknot lead to a drastic decrease (to $2 \%$ ) in -1 frameshifting. However in contrast to the rabbit reticulocyte lysate system, which strictly requires a pseudoknot, a predicted stem loop sequence was capable of stimulating a high level of -1 frameshifting in E. coli (Brierley et al., 1997). In the present in vivo system, we tested the effect of a pseudoknot on -1 frameshifting by removing the pseudoknot from the frameshifting cassette. Assuming no formation of an alternative secondary structure capable of inducing a pause, we report that still a significant proportion of ribosomes ( $30 \%)$ change the reading 
frame in the absence of pseudoknot, albeit fewer compared to the frameshifting construct (70\%) (Figure 6B).

One possible reason for the differences observed in frameshifting efficiencies is the use of different experimental systems and vector sequences. The viral IBV $1 a / 1 b$ system we utilized was altered to be suitable for translation in E. coli (stated in results). That makes a direct comparison of the previous experiments with the current in vivo work difficult. In addition, we cannot rule out possible influences of other cis-acting elements; i.e. in E. coli the SD-antiSD base pairing can influence efficiency of -1 frameshifting (Fayet and Prère, 2010). Larsen et al. reported that deletion of the 5 ' stimulatory SD element in dnaX decreased frameshifting from $56 \%$ to $26 \%$ (Larsen et al., 1994). In B. subtilis cdd frameshifting, synthesis of the frameshifting product strongly depends on a $5^{\prime}$ SD element; mutations caused $\sim 10$ fold decrease in frameshifting (Mejlhede et al., 1999). We note that the constructs used by Brierley et al. lacked the SD sequence upstream of the IBV $1 a / 1 b$ slippery site (which is present in our constructs) (Brierley et al., 1997) which could explain the differences. Together, our in vivo results support that -1 frameshifting relies on the coordinated action of more than one stimulatory element in E. coli, each of which can potentially act independently and up to varying levels.

The in vivo luciferase assay enabled us to test the functionality of the modified IBV $1 a / 1 b$ frameshifting site in E. coli translation system. However, genetic studies alone would not elucidate the precise mechanism of -1 frameshifting. In order to test and challenge different models proposed to explain frameshifting, we have systematically analyzed kinetics of different steps of the elongation cycle using the highly purified in vitro translation system developed in our lab. This provided direct information about the position and duration of ribosomal pause.

\subsection{Decoding at the frameshifting site}

Selection of the correct aminoacyl-tRNA is monitored at several stages to ensure production of correct peptides (Rodnina and Wintermeyer, 2001b). Previous studies proposed that frameshifting takes place during decoding when slippery codons are presented at the $P$ and A sites (Harger et al., 2002; Licznar et al., 2003; Plant and Dinman, 2005). Harger et al. 
proposed that peptidyl-transfer was involved in frameshifting, based on the results from Dinman et al. which showed antibiotics that slow down peptidyl transfer such as sparsomycin increased -1 frameshifting and anisomycin lead to decreased -1 frameshifting (Dinman et al., 1997). Indeed, sparsomycin facilitates spontaneous translocation in the absence of EF-G, therefore it was stated by Fredrick and Noller that the peptidyl-transferase center of 50S is involved in translocation (Fredrick and Noller, 2003). In addition, they stated mutants of eEF-1A but not of eEF-2 were related to increased -1 frameshifting (Harger et al., 2002). In fact, mutants of EF-G which affect -1 frameshifting were described (Ortiz et al., 2006). In addition, ribosomal mutations in helix 34 and 23 S rRNA that influence tRNA translocation were shown to increase spontaneous -1 frameshifting, which also supports a relationship between translocation and frameshifting (Kubarenko et al., 2006; Sergiev et al., 2005).

A mechanical explanation to frameshifting at the step of A-site tRNA accommodation was provided by Plant et al. in the $9 \AA$ model. They proposed that during accommodation the anticodon loop of the A-site tRNA moves by $9 \AA$ which pulls the mRNA with it while the downstream secondary structure creates tension by pulling the mRNA in the opposite direction. The tension created by the pseudoknot would be relieved by uncoupling of base pair interactions at the $A$ and $P$ sites and repairing one nucleotide in $5^{\prime}$ direction (Plant, 2003). Although the $9 \AA$ model explains how the pseudoknot would be involved in facilitating frameshifting, the movement of the anticodon loops from $A / T$ to $A / A$ sites was only $a$ prediction and not based on direct experimental results (Noller et al., 2002), therefore this part is questionable. Yet, it is not clear how the tension brought on the A-site tRNA would cause the uncoupling of codon:anticodon interactions and slippage on the P-site tRNA. In this model the GTP hydrolysis by the eEF-1A was suggested to lower the threshold of -1 frame decoding by the ribosome, however such an energetic input may also be provided by EF-G during translocation (Plant, 2003).

Many groups performed extensive peptide analysis at the frameshifting sites which pinpointed the last in-frame codon decoded (Jacks et al., 1988b; Sekine et al., 1992; Weiss et al., 1989; Yelverton et al., 1994). However, pre- and post-peptidyl transfer frameshifting mechanisms would actually result in incorporation of identical amino acids at the frameshifting codon. Therefore, it is not possible to distinguish whether tRNA slippage 
occurred before peptidyl transfer, i.e. during accommodation, or after peptidyl transfer, i.e. during translocation.

In this work, we were able to overcome the limitations of the previously used approaches by measuring the kinetics of amino acid incorporation at each step of decoding. Our results on ribosomes programmed with variants of the IBV $1 a / 1 b$ frameshift fragment with or without stimulatory elements suggest that decoding of the slippery codons was not altered when efficient frameshifting occurred. The first codon of the IBV $1 a / 1 b$ slippery site is UUA encoding Leu in the regular reading frame. We note that Leu was incorporated into the peptides more slowly than the preceding Tyr or the following amino acids. This might be explained by the observation that translocation of the initiator tRNA ${ }^{\text {fMet }}$, which is required to expose the Leu codon in the A site, is slower than of elongator tRNAs (Dorner et al., 2006). Translocation of tRNA ${ }^{\text {fMet }}$ would slow down the translocation of the P- and A-site tRNAs, thereby negatively affect the incorporation of the next amino acid moiety to the fMY dipeptides.

To challenge the hypothesis that frameshifting occurs during the decoding of the Lys codon at the A-site, we studied decoding of Lys. We show that the incorporation of Lys coded by the second codon of the slippery sequence was essentially unchanged regardless of the mRNA variant employed (Table 1), suggesting that no frameshifting occurs up to the step of Lys decoding and incorporation into the peptide. We also tested whether zero frame AAG or -1 frame AAA Lys codon was decoded by tRNA ${ }^{\text {Lys }}\left(U_{U} U^{*}\right)$ at the A-site. Although decoded by the same isoacceptor tRNA ${ }^{\text {Lys }}$, we note that Lys is decoded slightly slower on AAG codons compared to AAA codons (Table 2). This is in agreement with a previous study which compared the efficiency of dipeptide formation on both Lys codons by E. coli tRNA ${ }^{\text {Lys }}$ (OrtizMeoz and Green, 2010). Similarly, the rates of decoding of UUU and UUC codons by tRNA $^{\text {Phe }}(A A G)$ differ by a factor of two (Gromadski et al., 2006; Gromadski and Rodnina, 2004). The rate of Lys incorporation $\left(5 \mathrm{~s}^{-1}\right)$ in the frameshifting construct was similar to rate of Lys incorporation on AAG codons $\left(6.1 \mathrm{~s}^{-1}\right)$, whereas the rate on AAA codons was slightly faster $\left(8.6 \mathrm{~s}^{-1}\right)$, supporting that tRNA slippage occurs following decoding of Lys codon in $A$ site. 
Our experiments on decoding of lysine are contrasting with the models that proposed slippage during or immediately after the Lys accommodation (Harger et al., 2002; Plant, 2003; Plant and Dinman, 2005). Also it is worth mentioning, frameshifting before peptide bond formation is unlikely, because peptide bond formation takes place instantaneously once aminoacyl-tRNA is accommodated, the time window between the accommodation and peptide bond formation is rather narrow (Gromadski and Rodnina, 2004; Pape et al., 1998; Rodnina and Wintermeyer, 2001a).

It was previously shown that $E$. coli tRNA ${ }^{\text {Lys }}$ (UUU*) base pairs with different strengths with $A$ and $G$ nucleotides due to the modified (34U*) nucleotide $\mathrm{mnm}^{5} \mathrm{~S}^{2} \mathrm{U}$ (Murphy et al., 2004; Yarian et al., 2000; Yokoyama and Nishimura, 1995), and as a consequence of these interactions A site codon influences frameshifting (Tsuchihashi and Brown, 1992). We show that decoding of Lys is not effected in the A site; still we regard that the interactions of the modified $U^{*}\left(\mathrm{mnm}^{5} \mathrm{~S}^{2} \mathrm{U}\right)$ base with the $A$ and $\mathrm{G}$ bases of the Lys would be critical at the $P$ site. These results correlate with the previously published peptide sequencing data and support a post-peptidyl transfer slippage mechanism (Barak et al., 1996; Horsfield et al., 1995; Jacks et al., 1988b; Weiss et al., 1989; Yelverton et al., 1994).

\subsubsection{Role of tRNA competition}

Examining the rate of amino acid incorporation at the codon $3^{\prime}$ adjacent to the frameshifting site showed that translation was greatly slowed down when both the slippery sequence and the frameshifting pseudoknot were present. However, we note that Val in -1 frame was incorporated faster and more efficiently than Phe in the zero frame (Table 1, Figure 10). From the mechanistic point of view, there could be two potential explanations for the frameshifting mechanism. One possibility is that frameshifting may take place during the accommodation of the codon following the frameshift site. Alternatively, frameshifting could occur at a preceding step that is rate limiting for the next incorporation step.

As long as the cognate tRNA does not occupy the A site, there can be competition for binding at the A-site. Especially under starvation conditions, relative abundance of cognate isoacceptor tRNAs can influence translational errors and define which codon would be translated (Buchan and Stansfield, 2007). In case tRNA slippage occurs during the A-site 
accommodation step of the elongation cycle before the peptidyl transfer, the efficiency of frameshifting would depend on the availability of A-site substrates. Moreover, concentrations of aminoacyl-tRNAs coded by the zero and -1 frames should affect the frequency of recoding. Therefore, we questioned whether availability or concentrations of aminoacyl-tRNAs influence programmed -1 frameshifting.

In order to investigate whether the vacancy of the A site affects - 1 frameshifting, we have omitted either one of the potential A-site substrates, Phe-tRNA ${ }^{\text {Phe }}$ and Val-tRNA ${ }^{\text {Val }}$, which would bind to the A site in zero frame and -1 frame, respectively. Surprisingly, even in the absence of the zero frame A-site substrate Phe-tRNA ${ }^{\text {Phe }}$, there was no difference in the rate of -1 frame amino acid (Val) incorporation, indicating that frameshifting was not affected by the competition between tRNA ${ }^{\mathrm{Val}}$ and tRNA $^{\text {Phe }}$ (Figure 11, Table 3). We agree that nonprogrammed frameshifting events can be observed as a result of cognate-tRNA availability, however for programmed -1 frameshifting that is unlikely to be the driving factor.

Consistently, no difference in the zero frame (Phe) incorporation was observed when -1 frame competitor Val-tRNA ${ }^{\text {Val }}$ was excluded. Furthermore, there was no competition for Asite binding between the two tRNAs that base pair at -1 frame and zero frame. Thus, our results do not support models that invoke competition at the A site in frameshifting, for example those reporting by peptide sequence analysis that at higher concentrations of the out-of-frame aminoacyl-tRNA or due to starvation at the in-frame codon, efficiency of ribosome slippage and frameshifting increases (Barak et al., 1996; Kolor et al., 1993; Yelverton et al., 1994). We emphasize that competition at the A-site could be relevant in accidental frameshifting mechanisms.

We note that the scenario would be completely different in case of +1 frameshifting. During +1 frameshifting in the Ty1 element a rare arginine codon (AGG) is present at the slippery site which increases the likelihood of frameshifting. Increasing or decreasing the levels of Arg-tRNA ${ }^{\text {Arg }}$ reduces or induces +1 frameshifting (Gurvich et al., 2005). In addition, a vacant A site representing a Trp codon was reported to induce frameshifting in the RF2 gene in vivo up to $50 \%$, while induction of the $\mathrm{TRNA}^{\mathrm{Tr}}$ gene shifted the equilibrium back towards zero frame decoding (Sipley and Goldman, 1993). Apparently, different kinetic determinants have 
a role in +1 and -1 frameshifting; therefore, comparison of the mechanisms should be made with caution.

As shown here, programmed -1 frameshifting is not defined by the interactions at the decoding site on the slippery site or on the codon $3^{\prime}$ of the slippery site. These results allowed us to exclude the $9 \AA$ model of the -1 frameshifting, the simultaneous slippage model and the integrated model that proposed tRNA slippage before peptidyl transfer.

\subsubsection{Role of frameshifting elements}

Examining the rate and efficiency of amino acid incorporation at the overlapping codon with the complexes lacking the pseudoknot revealed that the presence of a slippery site was sufficient to stimulate decoding in -1 reading frame. This was in agreement with the in vivo experiments, where we observed $30 \%$ frameshifting efficiency (Figure 6). The rate of incorporation of the -1 frame substrate Val-tRNA ${ }^{\mathrm{Val}}$ was similar to the normal rate of Val incorporation in zero-frame (Table 1). The efficiency of the translation in -1 was also rather high, with more than one third of ribosomes moving to the alternative reading frame at the overlapping codon. This was surprising, because generally it is believed that frameshifting strongly depends on the presence of the pseudoknot in the IBV $1 a / 1 b$ fragment (Brierley et al., 1997). Mutational work in the RSV frameshifting sequence suggested that a strong slippery site may compensate for the destabilized pseudoknot, which is in line with our results (Nikolic et al., 2012).

It is generally accepted that secondary structures can act as roadblocks for the ribosomes and may impede translation (Lopinski et al., 2000; Somogyi et al., 1993; Tholstrup et al., 2012; Tu et al., 1992; Wen et al., 2008). Using mRNAs that were mutated at the slippery site, we tested the role of the pseudoknot on translation. The rates of Tyr, Leu and Lys incorporation was essentially the same in the presence and absence of the pseudoknot, while the codon following the Lys codon rate of decoding was greatly reduced (Table 1). In these complexes we reported incorporation at the -1 reading frame, albeit with slower rates $\left(0.09 \mathrm{~s}^{-1}\right.$ for Val; Table 1). This would indicate that a stimulatory sequence is not obligatory; however, the presence of it greatly enhances the efficiency of frameshifting. 
Previously, Garcia et al. compared the efficiencies of frameshifting signals from BWYV in vivo in E. coli and in vitro in rabbit reticulocyte lysate and wheat germ extracts (Garcia et al., 1993). They confirm that the response of $E$. coli ribosomes was less stringent to the viral frameshifting elements. They reported that mutations in the slippery site or the pseudoknot that completely abolished frameshifting on eukaryotic ribosomes, lead to a 30-50\% decrease in E. coli in the BWYV frameshifting site (Garcia et al., 1993), which is in line with the results presented in this study.

Our results show that in the absence of slippery site, a certain population of ribosomes would be forced to change the reading frame and in that case $\mathrm{G}: U$ (codon:anticodon) pairing at the first position would be tolerated, although it is unclear how this can be achieved. We speculate that the identity of the P-site tRNA might still play an important role determining the slippage efficiency. The SD sequence and the pseudoknot in combination might lead to frameshifting despite the lack of the slippery site. In addition frameshifting may have a different mechanism in the absence or presence of a slippery site. This unexpected result is in agreement with the previously suggested role for the pseudoknot in creating tension on the mRNA and forcing the ribosomes to shift the reading frame (Namy et al., 2006; Plant, 2003; Plant and Dinman, 2005).

Another interesting observation of this work was that after decoding the Lys codon, the structure of the pseudoknot might be affected compared to the preceding steps of

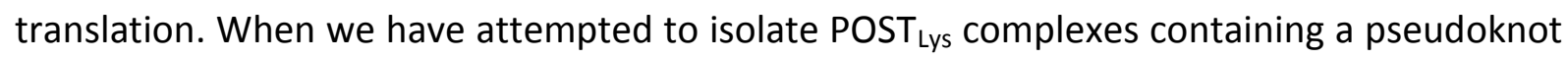
that had MYLK-tRNA ${ }^{\text {Lys }}$ in the $P$ site and a UUU codon in the A site (data not shown), those ribosomes failed to resume translation following a long pause upon addition of ternary complexes for Phe-tRNA ${ }^{\text {Phe }}$ and Val-tRNA ${ }^{\text {Val }}$. Previous heelprinting work indicated that the pseudoknot stem 1 region is melted while the ribosomes are paused over the slippery site (Tu et al., 1992). Possibly, during the time required for the complex purification, the halfmelted pseudoknot acquires an unusual topology that blocks factor binding and the following elongation step. However, POST Leu $_{\text {Complexes in which }} \mathrm{PMYL}$-tRNA $^{\text {Leu }}$ was in the P site could resume translation. This implies the tension or ribosomal contacts by the pseudoknot influence elongation provided that the spacer length with the $A$ site is less than 9 nucleotides (Brierley et al., 1992; Mazauric et al., 2009; Morikawa and Bishop, 1992). 


\subsection{Translocation of tRNA ${ }^{\text {Lys }}$ at 50 S subunit}

Our systematic analysis at the frameshift site revealed that frameshifting was not an A-site event and a step following peptide bond formation was responsible for -1 frameshifting. Therefore, we focused on the translocation of tRNA ${ }^{\text {Lys }}$. During translocation at the $50 \mathrm{~S}$ subunit the acceptor ends of the deacylated tRNA and peptidyl tRNA move from the A and P sites to the $\mathrm{P}$ and $\mathrm{E}$ sites. It was suggested that the dynamic nature of the ribosomes during translocation would allow change of the reading frame (Weiss et al., 1989).

The puromycin assay allows monitoring the reactivity of the $3^{\prime}$ ends of the acceptor end of the peptidyl-tRNA located in the 50S P site, whereas peptidyl-tRNA situated in the A site is essentially nonreactive with puromycin. By testing the reactivity of $\mathrm{FMYLK}$-tRNA ${ }^{\text {Lys }}$ complexes in real-time through the course of translation, we observed that $50 \mathrm{~S}$ translocation proceeded with the same rate on frameshifting and control complexes (Figure 14).

The dynamics of tRNA ${ }^{\text {Lys }}$ labeled with proflavin at the D loop was also monitored which reports on translocation (Rodnina et al., 1997; Savelsbergh et al., 2003). Consistent with the puromycin experiment, similar rates for translocation of tRNA ${ }^{\text {Lys }}$ (Prf) was observed with frameshifting or control complexes (Figure 15). This showed that the tRNA slippage must occur following the transfer of the $3^{\prime}$ end of fMYLK-tRNA ${ }^{\text {Lys }}$ to the $P$ site on the 50S subunit (because the $D$ loop is located at the $50 \mathrm{~S}$ subunit). Accordingly, it is unlikely that tRNAs would slip during formation of the hybrid states (Weiss et al., 1990). Indeed, while this thesis was in preparation, an exciting finding in our laboratory has shown that $50 \mathrm{~S}$ and $30 \mathrm{~S}$ translocation takes place simultaneously and the formation of hybrid states is not a prerequisite for EF-G binding (unpublished work Holtkamp et al.). However the concerted movement of the two tRNAs on both subunits can be uncoupled by antibiotics such as hygromycin B, spectinomycin and viomycin or by mutations in EF-G (Ermolenko et al., 2007; Modolell and Vazquez, 1977; Peske et al., 2004). Could a similar uncoupling of translocation on both subunits is possible during frameshifting? To find an answer we focused on EF-G dependent translocation on 30 s subunit. 


\subsection{EF-G binding, translocation of the anticodon ends}

The translocation step is intrinsically a very rapid process. On the other hand, a pause created by the pseudoknot may potentially slow down a certain elemental step of translocation and an otherwise transient intermediate might accumulate during frameshifting. Our results based on puromycin reactivity suggest that the POST state has been reached at the $50 \mathrm{~S}$ subunit with the same rates in frameshifting and control ribosomes. Next, we tested the last step of translocation which involves movement of the tRNAs and mRNA from $A$ to $P$ and $P$ to $E$ sites on the 30 S subunit (Moazed and Noller, 1989). On the $30 \mathrm{~S}$ subunit the movement of the tRNAs and mRNA is rapid and takes place in the unlocked state of the ribosome-EF-G complex (Munro et al., 2010; Rodnina and Wintermeyer, 2011; Savelsbergh et al., 2003). The binding of EF-G to the ribosome during translocation was reported by the fluorescence quenching of $\mathrm{L} 7 / 12$-labeled ribosomes by a quencher attached to EF-G. The recruitment of EF-G was not impaired during frameshifting as shown by similar rates of EF-G binding, $\sim 2 \mathrm{~s}^{-1}$, for control and frameshifting complexes. In contrast, EF-G dissociation, which can be monitored by the release of quenching, was slower by a factor of four from the frameshifting complexes compared to control ribosomes. With complexes containing only the pseudoknot (-/+), EF-G dissociation was as slow as with the frameshifting complexes, $\sim 0.5 \mathrm{~s}^{-1}$, but occurred only partially, as seen from the smaller amplitude compared to the frameshifting case (Figure 16).

These findings suggest that the tRNA ${ }^{\text {Lys }}$ slippage occurs during EF-G-induced unlockingrelocking rearrangements of the ribosome during translocation. The most likely explanation for the EF-G retention is that, in the presence of the pseudoknot the back swiveling movement of the $30 \mathrm{~S}$ subunit head with respect to the body is affected (Cunha, 2012; Savelsbergh et al., 2009).

We propose that the prolonged retention of EF-G on the ribosome may be caused by the inhibition of the 305 subunit head movement towards the rigid pseudoknot structure on the mRNA. When the slippery sequence is present, the ribosome may escape this mechanically trapped state by moving by less than three nucleotides at a time, which would lead to -1 frameshifting. In the absence of the slippery sequence but in the presence of the pseudoknot, EF-G is retained on the ribosome even more efficiently, because the ribosome 
cannot escape the trapped state by -1 slippage, which slows down the incorporation of the following amino acids in both zero and -1 frames.

We show for the first time on the complexes programmed with mRNA containing a slippery site and a pseudoknot that frameshifting occurs when EF-G is still bound on the ribosomes. This is in line with the structural study of Namy et al. (Namy et al., 2006) who proposed that the IBV pseudoknot would interfere with the translocase eEF-2. We should note however, that Namy et al. used elongating ribosomes with a pseudoknot, but in the absence of a slippery sequence (Namy et al., 2006). Such complexes - which are comparable to the complexes programmed with mRNA with a pseudoknot alone utilized in our study - are not authentic frameshifting complexes and differ from those in a number of important ways. Similarly, to the authentic frameshifting situation, the pseudoknot slows down translocation; however, the recoding is induced to a much smaller extent. The stalling effect of the pseudoknot is also in agreement with the IBV fragment heelprinting experiments, which stated a role for pausing while the ribosomes are positioned over the slippery site with the $P$ site codon approximately 8 to 12 nucleotides upstream of the pseudoknot (Kontos et al., 2001). However, our data show that ribosomes are able to shift to a different reading frame on the slippery site also in the absence of the pseudoknot. Therefore, although the mechanical pause brought by the pseudoknot to the system can drive frameshifting to significant levels, other interactions governed by the identity of tRNA ${ }^{\text {Lys }}$ are of crucial importance.

Based on the results of our kinetic analysis of programmed frameshifting on the IBV frameshifting site in E. coli we envisage that the ribosomes may have three fates upon encountering the frameshifting Lys codon (Figure 17).

(1) Ribosomes might pause and the tension would be relieved by changing the reading frame (Plant, 2003). This route preferred by most of the frameshifting complexes (about 70\%) in which the rate of -1 frame Val incorporation is faster than the rate of zero frame Phe incorporation. For the ribosomes that lack the slippery site chances to base pair in the -1 reading frame after reading the Lys codon are lower. Despite the lack of the slippery sequence, a significant population of ribosomes might still choose to change the reading frame, however at a very low rate, because in this case 
the P-site codon in the -1 frame is a near-cognate codon GAA codon. Dissociation of EF-G is possible only when certain conformational requirements are met. In the absence of the slippery site it is presumably harder to achieve, but despite the lack of the slippery site frameshifting is still a rescue pathway for the complexes to carry on translation. In the absence of pseudoknot, significant proportion (about 30\%) of ribosomes can change the reading frame indicating that -1 frame decoding can be almost as favorable as zero frame decoding.

(2) Ribosomes force the tRNAs back into the zero reading frame. In the presence of the pseudoknot, this path requires that tRNAs are translocated even slower, because a longer time is required to drive translocation opposing the tension created by the pseudoknot hindering the mRNA from opening the access to the next codon.

(3) Ribosomes encountering the pseudoknot might stop translating and an abortive peptide would be produced. This fate is pronounced in the absence of the slippery site, where almost half of the ribosomes quit translation in the POST Lys $_{\text {state following }}$ incorporation of Lys.

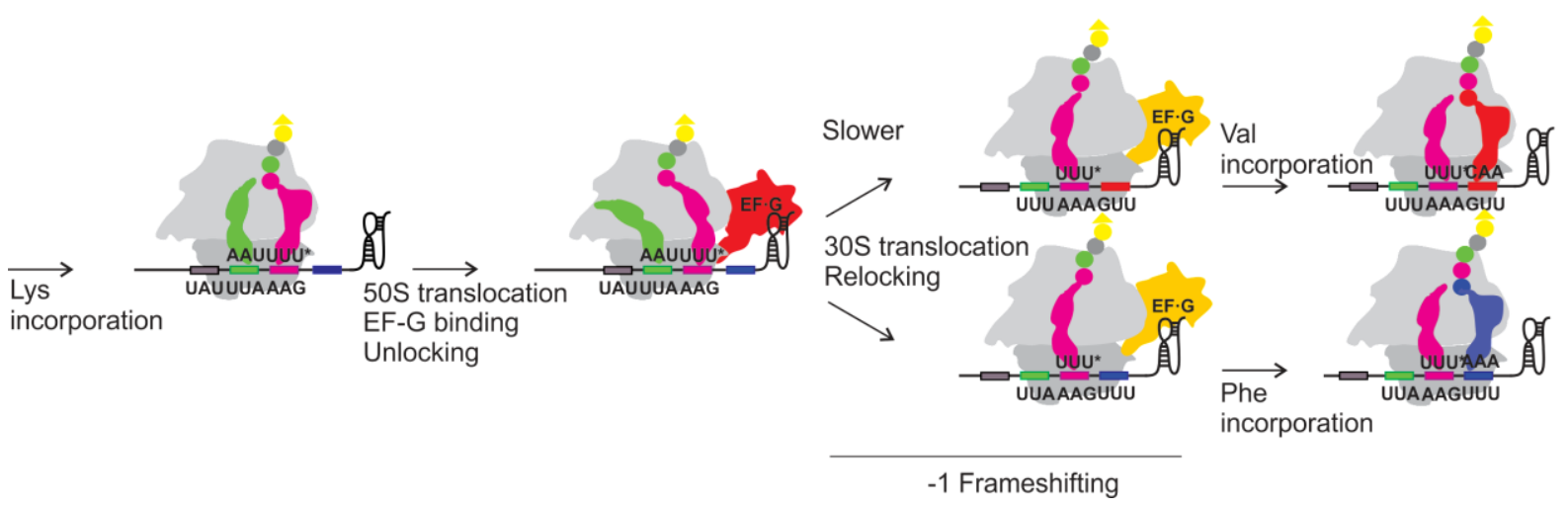

Figure 17. Proposed model for -1 frameshifting. The ribosomal subunits are represented in light grey (50S) and dark grey (30S). EF-G is depicted in red (GTP bound) and dark yellow (GDP bound), tRNAs for Leu in green, Lys in magenta, Val in red, Phe in blue.

Here we describe the detailed mechanism of -1 frameshifting based on the kinetic analysis of the elemental steps in real-time. Considering the variety of systems that use -1 frameshifting, it is possible that more than one mechanism evolved among prokaryotes and eukaryotes. The mechanism of -1 frameshifting may differ among the genes of the same 
organism. Although some similarities exist between +1 and -1 frameshifting events, the underlying mechanism with regard to the role of the stimulatory elements is likely completely different. A comprehensive insight into the mechanism of +1 frameshifting is still lacking, which would greatly increase our global understanding of frameshifting events and the sophisticated nature of the recoding events in the ribosome but also of reading frame maintenance in general. 


\section{MATERIALS AND METHODS}

\subsection{Chemicals and enzymes}

Unless otherwise stated all chemicals were purchased from Merck (Darmstadt, Germany).

Restriction enzymes were purchased from New England Biolabs (Frankfurt, Germany).

Antibiotics were purchased from Calbiochem (California, USA).

[14C]-labeled lysine

$[3 \mathrm{H}]$-labeled methionine

Alexa Fluor 488 C5-maleimide

Guanosine-5'-triphosphate (GTP)

L-amino acids

Scintillation liquid Lumasafe plus

Scintillation liquid Quickszint 361

Lysozyme from chicken egg white

Phenol aqua- RNA grade

Phosphoenol pyruvate

Phusion HF-DNA polymerase

QSY9-maleimide

\subsection{Buffers}

Buffer A
Hartmann (Braunschweig, Germany)

Hartmann (Braunschweig, Germany)

Invitrogen (Oregon, USA)

Sigma (Steinheim, Germany)

Sigma (Steinheim, Germany)

PerkinElmer (Massachusetts, USA)

Zinser analytic (Frankfurt, Germany)

Sigma (Steinheim, Germany)

Roth (Karlsruhe, Germany)

Roche (Mannheim, Germany)

NEB (Frankfurt, Germany)

Invitrogen (Oregon, USA)

$50 \mathrm{mM}$ Tris- $\mathrm{HCl}, \mathrm{pH} 7.5$

$70 \mathrm{mM} \mathrm{NH}_{4} \mathrm{Cl}$ 
$30 \mathrm{mM} \mathrm{KCl}$

$7 \mathrm{mM} \mathrm{MgCl} 2$

Buffer B

$50 \mathrm{mM}$ Tris- $\mathrm{HCl}, \mathrm{pH} 7.5$

$70 \mathrm{mM} \mathrm{NH}_{4} \mathrm{Cl}$

$30 \mathrm{mM} \mathrm{KCl}$

$14 \mathrm{mM} \mathrm{MgCl} 2$

Buffer C

20 mM Tris- $\mathrm{HCl}, \mathrm{pH} 8.5$

$300 \mathrm{mM} \mathrm{NaCl}$

5 mM 2-Mercaptoethanol

$15 \%$ glycerol

Buffer D

20 mM Tris- $\mathrm{HCl}, \mathrm{pH} 8.5$

$300 \mathrm{mM} \mathrm{NaCl}$

5 mM 2-Mercaptoethanol

$15 \%$ glycerol

$250 \mathrm{mM}$ imidazole

HPLC buffer A

$20 \mathrm{mM}$ ammonium acetate $\mathrm{pH} 5.0$

$10 \mathrm{mM}$ magnesium acetate

$400 \mathrm{mM} \mathrm{NaCl}$

HPLC buffer B

$20 \mathrm{mM}$ ammonium acetate $\mathrm{pH} 5.0$

$10 \mathrm{mM}$ magnesium acetate

$400 \mathrm{mM} \mathrm{NaCl}$ 
$15 \% \mathrm{EtOH}$

HPLC Buffer C for peptide analysis

HPLC Buffer D for peptide analysis

Labeling buffer (for EF-G)

2X RNA loading dye

5X DNA loading buffer

10X TAE
$0.1 \%$ TFA

$0.1 \%$ TFA

65\% Acetonitrile

$50 \mathrm{mM}$ Tris- $\mathrm{HCl}, \mathrm{pH} 7.5$

$70 \mathrm{mM} \mathrm{NH}_{4} \mathrm{Cl}$

$30 \mathrm{mM} \mathrm{KCl}$

$7 \mathrm{mM} \mathrm{MgCl}_{2}$

$300 \mathrm{mM} \mathrm{NaCl}$

20 mM EDTA pH 8.0

2 mM Tris- $\mathrm{HCl} \mathrm{pH} 7.5$

$8 \mathrm{M}$ urea

$20 \%$ glycerol

0.05\% (w/v) bromophenol blue

$0.05 \%(\mathrm{w} / \mathrm{v})$ xylene cyanol

$30 \%$ glycerol

5 mM EDTA, pH 8.0

$0.25 \%(w / v)$ bromophenol blue

$0.25 \%(w / v)$ xylene cyanol

$0.4 \mathrm{M}$ Tris- $\mathrm{HCl}$

$0.2 \mathrm{M}$ acetic acid 
10 mM EDTA, pH 8.4

10X TBE

$0.89 \mathrm{M}$ Tris- $\mathrm{HCl}$

$0.89 \mathrm{M}$ boric acid

25 mM EDTA, pH 8.3

LB

$10 \mathrm{~g} / \mathrm{l} \mathrm{NaCl}$

$10 \mathrm{~g} / \mathrm{l}$ tryptone

$5 \mathrm{~g} / \mathrm{l}$ yeast extract

Lysis buffer

10 mM Tris- $\mathrm{HCl}$ pH 8.0

1 mM EDTA

$5 \mathrm{mg} / \mathrm{ml} \mathrm{lysozyme}$

\subsection{Plasmids}

pET24a(+)

Novagen

pGem Luc

Promega

pRL

Promega

\section{$4.4 \quad$ E. coli strains}

NovaBlue

Novagen

Tuner

Novagen

lacZY deletion mutants of BL21

BL21 (DE3)

Novagen 


\subsection{Primers}

Generation of the linearized pET24a(+)

Forward 5`-TGAGATCCGGCTGCTAACAAAGCCC

Reverse 5`ATGTATATCTCCTTCTTAAAGTTAAACAAAATTATTTCTAG

Generation of Fluc insert

Forward: 5`-ATGGAAGACGCCAAAAACATAAAGAAAGGCCC

Reverse: 5 '-CAATTTGGACTTTCCGCCCTTCTTGGCC

Generation of Rluc insert

Forward: 5`-ATGACTTCGAAAGTTTATGATCCAGAACAAAGGAAACG

Reverse: 5`-TTATTGTTCATTTTTGAGAACTCGCTCAACGAACG

Generation of the linearized parental vector

Reverse: 5 '-CAATTTGGACTTTCCGCCCTTCTTGGCC

Forward: 5`-ATGACTTCGAAAGTTTATGATCCAGAACAAAGGAAACG

Generation of IBV $1 a / 1 b$ gene insert $300 \mathrm{bp}$ (pNC10) (also used for sequencing)

Forward: 5`-CAGCGGTCAGCGTTGTTTTAC

Reverse: 5`-GTTGTGTGGAATTGTGAGCGGAT

Primer for vector \#1

Forward: 5 `-AGATCTTCTATGACTTCGAAAGTTTATGATCCAGAACAAAG

Reverse: 5`-GCGAAACTTCAGATACATATGTATATCTCCTTGATTCTTAACAAG

Primer for vector \#2

Forward: 5`-ATGACTTCGAAAGTTTATGATCCAGAACAAAGGAAACG

Reverse: 5`-AGAAGATCTTATTACGGCTCGCTTTGCAAGGGGTAC

Primer for vector \#3

Forward: 5`-CTTGTTAAGAATCAAGGAGATATACATATGTATTTAAAGTTTCG 
Reverse: 5`CAATTTGGACTTTCCGCCCTTCTTGGCC

Primer for vector \#4

Forward: $5 `$-GATATACATATGTATTTAAAGTTTCGCTAATAAGATCTTCTATGACTTCGAAAG

Reverse: 5`CTTTCGAAGTCATAGAAGATCTTATTAGCGAAACTTTAAATACATATGTATATC

Primer for vector \#5

Forward: 5 `-TGTTAAGAATCAAGGAGATATACATATGTATCTGAAGTTTCGCGGGGTATCAG

Reverse: 5`-CTGATACCCCGCGAAACTTCAGATACATATGTATATCTCCTTGATTCTTAACA

\subsection{List of vectors}

A series of test vectors were generated that vary in their frameshifting elements. Nomenclature and the description of the vectors used for the in vivo luciferase assay are as follows:

Vector \#1 was designed as a control vector without the slippery site and the pseudoknot. The Rluc gene was cloned in zero frame.

Vector \#2 was designed as the frameshifting construct, which contained the slippery site and the pseudoknot. The Rluc gene was cloned in -1 frame; Rluc activity reports on -1 frameshifting.

Vector \#3 was the same as vector \#2, the Rluc gene was cloned in zero frame; Rluc activity reports on readthrough.

Vector \#4 was designed to examine the effect of slippery site alone on frameshifting. The Rluc gene was cloned in -1 frame. 
Table 4. Vectors used for the $I B V 1 a / 1 b$ in vivo luciferase assay. Nucleotides forming the slippery site are underlined.

\begin{tabular}{|c|c|c|c|}
\hline Vector & Recoding site & Pseudoknot & $\begin{array}{l}\text { Rluc } \\
\text { frame }\end{array}$ \\
\hline 1 & $5^{\prime} \ldots$ UAUUUGAAGUUUCGC ...3' & & 0 \\
\hline 2 & $5{ }^{\prime} \ldots$ UAUUUAAAGUUUCGC & GGGGUAUCAGUCAGGCUCGGCUGGUACCCCUUGCAAAGCGAGCCG...3' & -1 \\
\hline 3 & 5 '...UAUUUAAAGUUUCGC & GGGGUAUCAGUCAGGCUCGGCUGGUACCCCUUGCAAAGCGAGCCG...3' & 0 \\
\hline 4 & $5^{\prime} \ldots$ UAUUUAAAGUUUCGC....3' & & -1 \\
\hline
\end{tabular}

\subsection{Molecular biology methods}

\subsubsection{Plasmid construction}

Vectors used for in vivo experiments contain the genes for firefly luciferase (Fluc) and renilla luciferase (Rluc), that were flanked by linkers corresponding to the test sequences of the IBV $1 a / 1 b$ gene. The Fluc gene was used as an internal control, whereas the expression of Rluc gene was an indicator for frameshifting. The vector used for the experiments was $\operatorname{PET} 24 a(+)$. Fluc and Rluc genes were amplified by PCR from vectors pGem Luc and pRL, respectively. All PCR reactions were done using a high fidelity polymerase, Phusion. The $\mathrm{pET} 24 \mathrm{a}(+)$ vector was amplified by PCR and in subsequent cloning steps first the Fluc and then the Rluc gene were blunt-end ligated. In order to prevent vector relegation, the $5{ }^{`}$ end of the linear vector was dephosphorylated by Antartic Phosphatase treatment $(0.4 \mathrm{U} / \mu \mathrm{l}$ in $1 \mathrm{X}$ Antartic Phosphatase Buffer, NEB).

The IBV $1 a / 1 b$ gene fragment was cloned from the pTZ18 vector, which was kindly provided by Dr. V. Katunin. Cloning of the $\sim 100$ nucleotide long IBV insert was not straightforward, since the large part of the insert was the $65 \mathrm{nt}$ long pseudoknot segment. Therefore, initially the minimal IBV fragment was cloned as a larger fragment of $\sim 300$ nucleotides into the parental vector. Hereafter, the IBV fragment was trimmed from both ends by deletion mutagenesis. This vector was used as a template for site directed mutagenesis to generate different test constructs. 
PCR products were purified using the PCR Cleanup Kit (Macharey-Nagel) to ensure better ligation efficiency. The concentration and the purity of the products were assessed by measuring the absorption at $260 \mathrm{~nm}$, confirmed by using agarose gel electrophoresis on a $1 \%$ gel. All ligation reactions were done using the Quick Ligation Kit (NEB) according to the manufacturer's protocol.

\subsubsection{Site-directed mutagenesis}

The quick-change mutagenesis-primers were complementary oligonucleotides. The primers were 30-45 nucleotides long, had a melting temperature of $\geq 78^{\circ} \mathrm{C}$, a GC content of $\sim 40 \%$ and ended with one or more $\mathrm{C}$ or $\mathrm{G}$ nucleotides. Mutations were in the middle of the primers and had 10-15 complementary nucleotides at both ends. The synthesis of the oligonucleotides was done commercially (Eurofins MWG Operon). PCR reactions for sitedirected mutagenesis were set as outlined in Table 5.

Table 5. PCR reaction conditions.

\begin{tabular}{lll}
\hline & Stock & Final \\
\hline Template & $\ldots \ldots$ & $250 \mathrm{ng}$ \\
5X HF Buffer & $5 \mathrm{X}$ & $1 \mathrm{X}$ \\
dNTP, each & $10 \mathrm{mM}$ & $0.2 \mathrm{mM}$ \\
Primer 1 & $4 \mu \mathrm{M}$ & $0.5 \mu \mathrm{M}$ \\
Primer 2 & $4 \mu \mathrm{M}$ & $0.5 \mu \mathrm{M}$ \\
DMSO & $100 \%$ & $3 \%$ \\
Phusion pol. & $2 \mathrm{U} / \mu \mathrm{l}$ & $0.02 \mathrm{U} / \mu \mathrm{l}$ \\
\hline
\end{tabular}

PCR reactions were performed in a preheated PeqStar thermocycler (Peqlab) according to the parameters given in Table 6 . In order to digest the methylated template DNA, the restriction enzyme $D p n$ I (20 units) was added after the PCR reactions and incubated for 1.5 $\mathrm{h}$ at $37^{\circ} \mathrm{C}$. An aliquot $(5 \mu \mathrm{l})$ of the $D p n$ I digested PCR product was run on an agarose gel (1\%) in order to confirm complete digestion. 
Table 6. PCR cycle conditions.

\begin{tabular}{lll}
\hline Cycles & Temperature & Time \\
\hline 1 & $98^{\circ} \mathrm{C}$ & $2 \mathrm{~min}$ \\
$2-25$ & $98^{\circ} \mathrm{C}$ & $20 \mathrm{~s}$ \\
& $60^{\circ} \mathrm{C}$ & $40 \mathrm{~s}$ \\
& $72^{\circ} \mathrm{C}$ & $15 \mathrm{~s} / \mathrm{kb}$ plasmid \\
26 & $72^{\circ} \mathrm{C}$ & $10 \mathrm{~min}$ \\
\hline
\end{tabular}

\subsubsection{Transformation}

Ligation products or quick-change PCR products were transformed into NovaBlue competent cells. Heat shock transformation was performed according to the manufacturer's protocol. Following transformation, the cells were plated kanamycin (Kan)-containing LB-agar plates and incubated at $37^{\circ} \mathrm{C}$ overnight. Antibiotic resistant transformants were selected and single colonies were inoculated in $5 \mathrm{ml}$ LB medium. On the following day, the cells were harvested at $10,000 \mathrm{~g}$ for $2 \mathrm{~min}$. Cell pellets were used for mini-preparation of plasmid DNA.

For the dual luciferase assay, test plasmids (100 ng each) were transformed into $10 \mu$ l Tuner cells. Heat shock transformation was performed as described in the producer's manual.

\subsubsection{Plasmid DNA preparation}

Plasmids were purified using the Macharey-Nagel Plasmid Preparation Kit (Mini, Midi or Maxi-scale) according to the manufacturer's instructions. The concentration and the quality of the DNA were checked by agarose gel electrophoresis and spectroscopic measurements at $260 \mathrm{~nm}$ (NanoDrop). All plasmids were verified by sequencing (Seqlab- Sequence Laboratories, Göttingen).

\subsection{In vivo dual luciferase assay}

In vivo luciferase assay of -1 frameshifting was performed by expressing different vectors (Table 4) in E. coli Tuner cells. Expression of T7 RNA polymerase in Tuner cells (lacZY deletion 
mutants of BL21) can be induced by addition of isopropyl- $\beta$,D-thiogalactopyranoside (IPTG). The lac permease mutation allows uniform entry of IPTG to all cells in the culture, which allows homogenous level of induction.

E. coli Tuner cells freshly transformed with the test plasmids were inoculated in $20 \mathrm{ml}$ LB medium supplemented with kanamycin $(30 \mathrm{mg} / \mathrm{ml})$. Following overnight growth the cultures were diluted 20X in fresh LB growth medium to reach a starting optical density 0.05 at 600 $\mathrm{nm}\left(\mathrm{OD}_{600}\right)$. Cells were grown at $37^{\circ} \mathrm{C}$ until the cultures reach mid-log phase. IPTG (70 $\left.\mu \mathrm{M}\right)$ was added $\left(\sim 0.5 \mathrm{OD}_{600}\right)$ to induce gene expression. The cells were harvested after incubation for $30-45 \mathrm{~min}$ at $37^{\circ} \mathrm{C}$ at $200 \mathrm{rpm}$. For harvesting, five $1 \mathrm{ml}$ samples from each culture were collected, centrifuged for $2 \mathrm{~min}$ at $10,000 \mathrm{~g}$ and the supernatant was removed. The cells were immediately frozen at $-20^{\circ} \mathrm{C}$ for short term or at $-80^{\circ} \mathrm{C}$ for long term storage.

Before every experiment, the cells were thawed on ice and resuspended in lysis buffer. The amount of the lysis buffer added to the cells varies to have equal amount of cells (1 $\mathrm{OD}_{600} / \mathrm{ml}$ ) for each sample. The cells were lysed on ice for $10 \mathrm{~min}$, then immediately frozen in liquid nitrogen. Lysates were thawed in $37^{\circ} \mathrm{C}$ waterbath for $5 \mathrm{~min}$ and the cell debris was removed by centrifugation for $5 \mathrm{~min}$ at $4^{\circ} \mathrm{C}$. The supernatant, which contained the Fluc-Rluc fusion protein, was used for expression analysis.

The dual luciferase assay was carried out in two steps. First, to measure firefly luciferase expression, $5 \mu \mathrm{l}$ of the supernatant were mixed with $100 \mu \mathrm{l}$ Bettle Juice (PJK GmbH), incubated at RT for $5 \mathrm{~min}$. The relative luciferase activity was measured in a luminometer (Sirius Single, PJK GmbH) with a delay time of 2 seconds and an integration time of 5 seconds.

Second, the renilla luciferase activity was measured by mixing $5 \mu$ l of cell extract with $100 \mu \mathrm{l}$ of Renilla Glow Juice (PJK GmbH). The samples were incubated for 0-20 min at RT followed by measurement of the luciferase activities. The same delay and measurement parameters were applied as for the firefly luciferase measurements.

The method of Grentzmann et al. was used to calculate the frameshifting efficiencies. Activity ratio of Rluc to Fluc gene of the test vectors (2-4, listed in Table 4) were divided by the activity ratio of control vector (1) and multiplied by 100 (Grentzmann et al., 1998a). 


$$
\text { Translation efficiency, } \%=\frac{\text { Rluc test } / \text { Fluc test }}{\text { Rluc control/Fluc control }} \cdot 100
$$

In every experiment each test construct was measured in triplicates. The experiments were repeated at least 3 times for error calculations.

\subsection{Preparation of labeled ribosomes (L7/12 138Alx488) and EF-G (WT and 209QSY9)}

For labeling of the residue 138 of L7/12 protein, Alexa Fluor 488 C5-maleimide dye ( $1 \mathrm{mg}$ ) was dissolved in buffer $A$ and mixed with $L 7 / 12$ protein $(\sim 80,000$ pmol, a generous gift from C. E. Cunha). The protein was labeled at $4^{\circ} \mathrm{C}$ overnight under rotation. Labeled $\mathrm{L} 7 / 12$ protein was purified by gel filtration on a HiLoad Superdex S75 10/300 column (GE Healthcare) equilibrated with buffer $A$ with a flow rate of $0.5 \mathrm{ml} / \mathrm{min}$. Fractions were collected and analyzed by SDS-PAGE for purity. The concentration and yield of the labeled $L 7 / 12$ protein was determined from absorbance measurements at $205 \mathrm{~nm}\left(\varepsilon=31 \mathrm{M}^{-1} \mathrm{~cm}^{-1}\right)$ (Scopes, 1974) and $495 \mathrm{~nm}\left(\varepsilon=71,000 \mathrm{M}^{-1} \mathrm{~cm}^{-1}\right)$.

70S ribosomes depleted from the $\mathrm{L} 7 / 12$ protein were used for reconstitution of $\mathrm{L} 7 / 12$ 138Alx488 labeled ribosomes. Removal of $L 7 / 12$ protein was carried out by $A$. LehwessLitzmann according to Mohr et al. (Mohr et al., 2002). Fivefold excess of labeled L7/12 protein was added to depleted ribosomes (cores) and incubated for $30 \mathrm{~min}$ at $37^{\circ} \mathrm{C}$. For removal of excess labeled $\mathrm{L} 7 / 12$ protein, ribosomes were loaded on $1.1 \mathrm{M}$ sucrose cushion in buffer $A$ and centrifuged in a TLS 55 swing out rotor in a Beckman Optima XP ultracentrifuge at $4^{\circ} \mathrm{C}$ and $259,000 \mathrm{~g}$ for $2 \mathrm{~h}$. Pellets were dissolved in buffer A. Aliquots (10 $\mu \mathrm{l}$ each) were prepared after determining the concentration of the $L 7 / 12$ labeled ribosomes. The aliquots were flash frozen in liquid nitrogen and stored at $-80^{\circ} \mathrm{C}$.

EF-G proteins (wild-type and $209 \mathrm{C}$ mutant) were overexpressed in BL21(DE3) cells at $37^{\circ} \mathrm{C}$ by Michael Zimmermann. Cells were harvested and pellets were resuspended in Buffer C supplemented with Complete Protease Inhibitor (Roche). Cells were lysed using an Emulsiflex apparatus and centrifuged at $300,000 \mathrm{~g}$ for $30 \mathrm{~min}$ (Ti50.2 rotor). The supernatant was loaded on a Protino column (Macharey- Nagel) for affinity chromatography using the Cterminal His-tag. The column was washed with buffer C, and eluted with buffer D. The eluted 
wild type EF-G protein was concentrated and the buffer was exchanged to $2 X$ buffer $A$ by membrane filtration (Vivaspin 10,000 MWCO); for storage, glycerol (1 volume) was added.

For the mutant EF-G 209C, three natural cysteine residues of wild-type EF-G were mutated and position 209 was exchanged to cysteine. For the labeling of EF-G 209C, initially buffer D was exchanged into labeling buffer using membrane concentrators (Vivaspin 30,000 MWCO). Labeling of EF-G 209C with the amine quencher dye, QSY9 was done by incubating fivefold excess of QSY9-maleimide dye with EF-G 209C protein in labeling buffer in the dark at $4^{\circ} \mathrm{C}$ overnight on a $360^{\circ}$ rotating shaker at $7 \mathrm{rpm}$. The dye-protein mix was applied to a Protino gravity-flow column (Macharey Nagel) for affinity purification of EF-G 209QSY9 using the $\mathrm{C}$-terminal His-tag. The resin was washed with 8 bed volumes of buffer $\mathrm{C}$ and the protein was eluted with Buffer D. Fractions were collected, presence of the labeled EF-G in the fractions was checked by SDS-PAGE. The eluted protein was concentrated and buffer was exchanged to $2 \mathrm{X}$ buffer $\mathrm{A}$ by membrane filtration (Vivaspin 10,000 MWCO), for storage glycerol ( 1 volume) was added. Aliquots $(30 \mu \mathrm{l})$ of the protein were flash frozen in liquid nitrogen and stored at $-80^{\circ} \mathrm{C}$. Concentration of the labeled EF-G QSY9 was calculated from absorbance at $280 \mathrm{~nm}$ assuming an extinction coefficient of $64,300 \mathrm{M}^{-1} \mathrm{~cm}^{-1}$ (Nguyen et al., 2010).

\subsection{RNA biochemistry methods}

\subsubsection{Aminoacylation of tRNAs}

Val-tRNA $^{\text {Val }}$ and Tyr-tRNA ${ }^{\text {Tyr }}$ were kindly provided by Dr. V. Katunin. tRNAs for Leu, Lys and Phe were prepared from E. coli total tRNA (Roche) and purified by gel filtration (Gromadski and Rodnina, 2004; Pape et al., 1998; Rodnina et al., 1994). Aminoacylation of tRNAs was performed in buffer A supplemented with ATP (3 mM), DTT (2 mM), tRNA (20 $\mu \mathrm{M})$, amino acid $(60 \mu \mathrm{M})$ and S100 $(2 \% \mathrm{v} / \mathrm{v})$ or aa-tRNA synthetase $(1 \% \mathrm{v} / \mathrm{v})$ for $1 \mathrm{~h}$ at $37^{\circ} \mathrm{C}$. After the reaction was completed the tRNAs were 3-4 times phenolized ( 1 volume) and the aqueous phase containing the tRNA was precipitated with 2.5 volume of ethanol for $1 \mathrm{~h}$ at $-20^{\circ} \mathrm{C}$, centrifuged at $5,000 \mathrm{~g}$ at $4^{\circ} \mathrm{C}$ for $1 \mathrm{~h}$. Precipitation was repeated twice to remove all traces of salts. Aminoacyl-tRNA pellets were vacuum dried to remove ethanol using a SpeedVac apparatus (Thermo Fisher). 


\subsubsection{Purification of tRNAs}

The aminoacylated tRNAs were enriched using high pressure liquid chromatography (Doerfel et al., 2013; Milon et al., 2007). Pellets of aminoacylated tRNAs were dissolved in $200 \mu \mathrm{l}$ HPLC high salt buffer A and applied to a LiChrosper WP 300 RP18 HPLC column $(250 \times 10.5$ $\mu \mathrm{m}$, Merck) pre-equilibrated with the same buffer. Chromatography was performed with a gradient of $0-40 \%$ HPLC buffer B at a flow rate of $3 \mathrm{ml} / \mathrm{min}$. Fractions were collected on ice $(6$ $\mathrm{ml}$ ) and $10 \mu$ aliquots were taken for scintillation counting using LumaSafe scintillation liquid $(2 \mathrm{ml})$ and/or by spectroscopic measurement where non-radioactive amino acids were used. Fractions containing the aminoacylated tRNA species were pooled and precipitated with ethanol at $-20^{\circ} \mathrm{C}$. tRNAs were dissolved in $5 \mathrm{mM} \mathrm{KOAc}$ and stored in aliquots $(40 \mu \mathrm{l})$ at $-80^{\circ} \mathrm{C}$. Aminoacylated tRNAs were tested in a peptide bond formation assay and found to be fully active.

\subsubsection{Labeling of tRNA ${ }^{\text {Lys }}$ with proflavin}

Proflavin-labeled tRNA ${ }^{\text {Lys }}$ was prepared in two steps. First, the reduction of the dihydrouridine at position 16-17 found at the D loop of the tRNA by borohydrate treatment. Second, proflavin was attached to these moieties (Wintermeyer and Zachau, 1979). tRNA ${ }^{\text {Lys }}$ (10 $\mathrm{A}_{260}$ units $/ \mathrm{ml}$ ) was dissolved in Tris- $\mathrm{HCl}\left(0.2 \mathrm{M}, \mathrm{pH}\right.$ 7.5). $\mathrm{NaBH}_{4}$ solution (100 mg dissolved in $1 \mathrm{ml}$ of $10 \mathrm{mM} \mathrm{KOH}$ ) was added to tRNA ${ }^{\text {Lys }}$ to a final concentration of $10 \mathrm{mg} / \mathrm{ml}$ and incubated for $30 \mathrm{~min}$ at $0^{\circ} \mathrm{C}$ in the dark. The reaction was stopped by the addition of acetic acid (100\%) to $\mathrm{pH} 4$ to 5 , and the tRNA was precipitated by addition of $0.3 \mathrm{M} \mathrm{KOAc},(\mathrm{pH} 4.5)$ and ice cold ethanol ( 2 volumes). After dissolving the pellet in $0.3 \mathrm{M} \mathrm{KOAc}$ ethanol precipitation was repeated twice to ensure removal of borohydrate.

For proflavin labeling reduced tRNA ${ }^{\text {Lys }}\left(10 \mathrm{~A}_{260}\right.$ units $\left./ \mathrm{ml}\right)$ was mixed with proflavin $(3 \mathrm{mM})$ in $0.1 \mathrm{M}$ sodium acetate $\left(\mathrm{pH} \mathrm{4.3)}\right.$. After incubation for $2 \mathrm{~h}$ at $37^{\circ} \mathrm{C}$ in dark, the reaction was stopped by the addition of $1 \mathrm{M}$ Tris- $\mathrm{HCl}(\mathrm{pH} 9)$, and the $\mathrm{pH}$ was adjusted to 7.5. Phenol extraction was performed to remove unreacted proflavin until the phenol phase was colorless. Ethanol precipitation was repeated three times. Incorporation of proflavin was quantified by absorbance measurements at $260 \mathrm{~nm}$ and $460 \mathrm{~nm}$. For fully labeled tRNA, the A460:A260 ratio is 0.055 (Milon et al., 2007). 


\subsection{4 mRNA transcription}

The mRNAs used in this work had the following sequences and properties:

+ Slippery site, + Pseudoknot

5' GGG AAU UCA AAA AUU GUU AAG AAU UAA GGA GAU AUA CAU AUG UAU UUA AAG UUU CGC GGG GUA UCA GUC AGG CUC GGC UGG UAC CCC UUG CAA AGC GAG CCG UAA UAA 3'

+ Slippery site, - Pseudoknot

5' GGG AAU UCA AAA AUU GUU AAG AAU UAA GGA GAU AUA CAU AUG UAU UUA AAG UUU CGC UAA UAA 3'

- Slippery site, - Pseudoknot

5' GGG AAU UCA AAA AUU GUU AAG AAU UAA GGA GAU AUA CAU AUG UAU UUG AAG UUU CGC A 3'

- Slippery site, - Pseudoknot, Val in zero frame

5' GGG AAU UCA AAA AUU GUU AAG AAU UAA GGA GAU AUA CAU AUG UAU UUG AAG GUU UCG CA 3'

- Slippery site, + Pseudoknot

5' GGG AAU UCA AAA AUU GUU AAG AAU CAA GGA GAU AUA CAU AUG UAU UUG AAG UUU CGC GGG GUA UCA GUC AGG CUC GGC UGG UAC CCC UUG CAA AGC GAG CCG UAA UAA 3'

- Slippery site, - Pseudoknot, Lys AAA codon

5' GGG AAU UCA AAA AUU GUU AAG AAU UAA GGA GAU AUA CAU AUG UAU UUG AAA GUU UCG CA 3'

mRNAs were produced by in vitro transcription using T7 RNA polymerase. Restriction digested plasmid DNA was used as template for run-off transcription. Plasmid DNA (3-5 mg) was digested with the restriction endonuclease BgIII (2000 units) in 1X buffer III (NEB) for 3 hours at $37^{\circ} \mathrm{C}$. Complete digestion of the plasmids was confirmed by agarose gel 


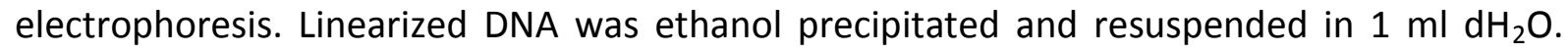
The reaction mix scheme for the transcription reaction is outlined in Table 7.

Table 7. Transcription reaction conditions.

\begin{tabular}{lll}
\hline & Stock & Final \\
\hline Transcription buffer & $5 \mathrm{X}$ & $1 \mathrm{X}$ \\
DTT & $100 \mathrm{mM}$ & $10 \mathrm{mM}$ \\
NTPs & $20 \mathrm{mM}$ & $3 \mathrm{mM}$ \\
GMP & $100 \mathrm{mM}$ & $5 \mathrm{mM}$ \\
Template DNA & & $100.0 \mu \mathrm{g} / \mathrm{ml}$ \\
Pyrophosphatase & $0.5 \mathrm{U} / \mu \mathrm{l}$ & $0.005 \mathrm{U} / \mu \mathrm{l}$ \\
T7 RNA Polymerase & $200 \mathrm{U} / \mu \mathrm{l}$ & $1.6 \mathrm{U} / \mu \mathrm{l}$ \\
RNase inhibitor & $50 \mathrm{U} / \mu \mathrm{l}$ & $0.2 \mathrm{U} / \mu \mathrm{l}$ \\
\hline
\end{tabular}

The transcription reactions were incubated for 3-4 hours and every hour samples were taken for analysis on an $8 \mathrm{M}$ urea PAGE. A representative gel is shown in Figure 18.

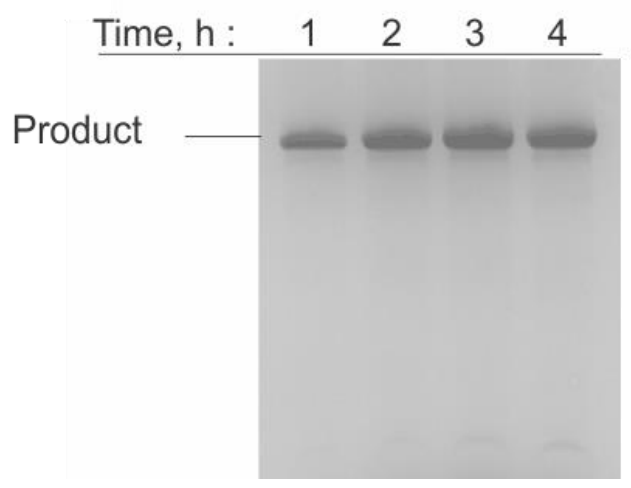

Figure 18. Time course of in vitro transcription of synthetic mRNAs. Transcription samples were collected every hour and analyzed on an $8 \mathrm{M}$ urea-PAGE. The gel was stained by methylene blue for visualization of the RNA.

\subsubsection{Purification of mRNAs}

In vitro transcribed mRNAs were purified to remove salts and enzymes. For analytical and preparative scale purifications, RNeasy Midi and Maxi Prep Kits (Qiagen) were used. mRNAs 
were purified according to the manufacturer's protocols except for the preparative scale samples, mRNAs were eluted three times with $1 \mathrm{ml}$ RNase-free water.

In order to check the quality, $5 \mu \mathrm{l}$ of the purified mRNA transcripts were loaded on an $8 \mathrm{M}$ urea PAGE. mRNA concentrations were determined from absorbance measurements at 260 $\mathrm{nm}$ using a NanoDrop instrument (Thermo Scientific) (for ssRNA, $\varepsilon_{\mathrm{A}}=15,400 \mathrm{M}^{-1} \mathrm{~cm}^{-1}$, $\varepsilon_{\mathrm{G}}=13,700 \mathrm{M}^{-1} \mathrm{~cm}^{-1}, \varepsilon_{\mathrm{C}}=9,000 \mathrm{M}^{-1} \mathrm{~cm}^{-1}$ and $\left.\varepsilon_{\mathrm{U}}=10,000 \mathrm{M}^{-1} \mathrm{~cm}^{-1}\right)$. To estimate the yield, the molecular weight of the oligos was calculated by the formula below:

$$
M . W .=(A n * 329.2)+(U n * 306.2)+(C n * 305.2)+(G n * 345.2)+159
$$

\subsection{Kinetic measurements}

\subsubsection{Initiation of the 705 ribosomes}

Ribosomes from E. coli MRE600, initiation factors (IF1, IF2 and IF3) and f[ $\left.{ }^{3} \mathrm{H}\right]$ Met-tRNA ${ }^{\text {Met }}$ were prepared as described previously (Gromadski and Rodnina, 2004; Milon et al., 2007; Rodnina et al., 1995; Rodnina et al., 1999).

Initiation complexes were prepared by incubating $E$. coli ribosomes $(1 \mu \mathrm{M})$ with mRNA (3 $\mu \mathrm{M})$, initiation factors IF1, IF2 and IF3 $(1.5 \mu \mathrm{M}), \mathrm{f}\left[{ }^{3} \mathrm{H}\right] \mathrm{M}^{\mathrm{M}}$ et-tRNA ${ }^{\mathrm{fMet}}(1.5 \mu \mathrm{M})$ and GTP $(1 \mathrm{mM})$ in buffer $\mathrm{A}$ up to $60 \mathrm{~min}$ at $37^{\circ} \mathrm{C}$. In order to monitor $70 \mathrm{~S}$ initiation complex formation, aliquots of $10 \mathrm{pmol}$ were taken at times $0,5,15,30,45$ and $60 \mathrm{~min}$ and filtered through a $0.45 \mu \mathrm{M}$ nitrocellulose filter (Sartorius, Germany) on a vacuum unit. Filters were washed with $4 \mathrm{ml}$ buffer $\mathrm{A}$ and were dissolved in $10 \mathrm{ml}$ Quickszint 361 scintillation liquid. Radioactivity in the samples was counted using a Tri-Carb 3110 TR instrument (Perkin Elmer). The efficiency of 705 initiation complex formation was estimated as follows:

Efficiency of 70 S IC formation, \% = Radioactivity $\left[{ }^{3} \mathrm{H}\right]_{\text {measured }}(\mathrm{dpm}) * 100 /$ Radioactivity $\left[{ }^{3} \mathrm{H}\right]_{\text {estimated }}\left(\mathrm{pmol} 70 \mathrm{~S}\right.$ ribosomes * specific activity of fMet tRNA $\left.{ }^{\mathrm{fMet}}(\mathrm{dpm} / \mathrm{pmol})\right)$

In preparative scale purification, initiation complexes (up to 2000 pmol) were pelleted through a $1.1 \mathrm{M}$ sucrose cushion in buffer $\mathrm{A}$. Centrifugation was performed in a TLS 55 
swing-out rotor in an ultracentrifuge (Beckman Optima XP) at 259,000 $\mathrm{g}$ at $4^{\circ} \mathrm{C}$, for $2 \mathrm{~h}$. Pellets were dissolved in buffer $A$. The concentration of the ribosomes was determined by absorbance measurements at $260 \mathrm{~nm}$, assuming $23 \mathrm{pmol}$ of $70 \mathrm{~S}$ ribosomes per $A_{260}$ unit. The radioactivity associated with $70 \mathrm{~S}$ ribosomes was measured by liquid scintillation counting to calculate efficiency of 705 initiation.

In experiments with labeled ribosomes (L7/12 138Alx488) initiation complexes were formed as described above, except the complexes were used directly without purification.

\subsubsection{Ternary complex formation}

EF-Tu was prepared as described previously (Gromadski and Rodnina, 2004; Rodnina et al., 1999). Ternary complexes composed of EF-Tu.GTP.aa-tRNA, were prepared by incubating EFTu (wild type his- tagged, used two fold excess over aminoacylated tRNA) with GTP (1 mM), phosphoenol pyruvate $(3 \mathrm{mM})$, and pyruvate kinase $(0.1 \mathrm{mg} / \mathrm{l})$ in buffer $A$ for $15 \mathrm{~min}$ at $37^{\circ} \mathrm{C}$, followed by the addition of $3 \mu \mathrm{M}$ of each aminoacylated tRNA. The aminoacyl-tRNA species were as follows; Leu-tRNA ${ }^{\text {Leu }}$, Tyr-tRNA ${ }^{\text {Tyr }},\left[{ }^{14} C\right]$ Lys-tRNA ${ }^{\text {Lys }}$, Phe-tRNA ${ }^{\text {Phe }}$ and Val-tRNA ${ }^{\text {Val }}$. Various ternary complexes with one or more aminoacyl-tRNA species were formed depending on the experiment (figure legends for details).

\subsubsection{Kinetics of amino acid incorporation measured by quench flow}

The quench-flow method was employed to measure the kinetics of amino acid incorporation during translation of different mRNA constructs. In a basic quench-flow experiment, two reactants are mixed with the help of a drive motor, which helps to move the reactants into a mixer. After the given time interval the drive motor is fired to expel the reactants through another mixer where the chemical quencher- a concentrated base in this case- is added to the reactants through middle drive syringe and the sample is pushed through the exit line into a collector for downstream analysis by means of analytical methods like HPLC. Basic scheme of a quench-flow apparatus can be seen in Figure 19. 


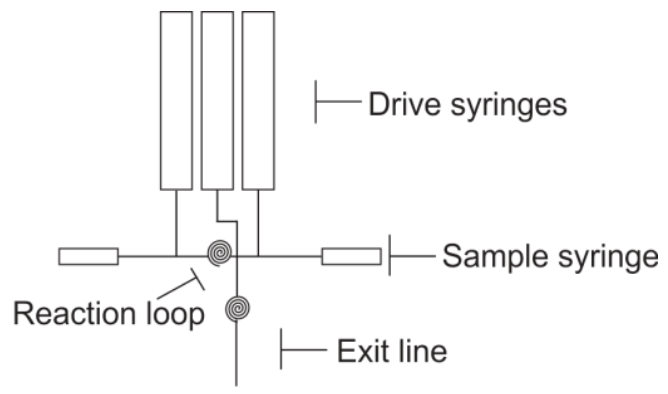

Figure 19. Scheme of a quench-flow apparatus. Buffer is loaded in the left and right drive syringes, middle drive syringe is used for the quencher (or for puromycin in the double mixing experiment described below). Equal volume of samples are loaded from the sample syringes and mixed at the reaction loops with varying sizes for the defined time intervals. Second mixer is shown before the exit line to depict the mixing with the quencher (or puromycin in the double mixing experiment). From there the sample passes through an exit line and is expelled into a collection tube.

Purified 70S ribosome complexes, programmed with model mRNAs, were diluted to $0.2 \mu \mathrm{M}$ in buffer A. Ribosome complexes were reacted with equal volumes $(14 \mu \mathrm{l})$ of ternary complexes, EF-Tu.GTP.aa-tRNA (1.5 $\mu \mathrm{M})$ for Tyr, Leu, Lys, Val and Phe in the presence of EF$\mathrm{G}(2 \mu \mathrm{M})$ in a quench-flow apparatus (KinTek) at $37^{\circ} \mathrm{C}$ in buffer $\mathrm{A}$. Reactions were quenched with $\mathrm{KOH}(0.8 \mathrm{M})$ at various time points. Peptides were released by hydrolysis at $37^{\circ} \mathrm{C}$ for 30 min, subsequently, the $\mathrm{pH}$ of the samples was neutralized by the addition of $1 / 10$ volume glacial acetic acid. The samples were centrifuged at 13,000 rpm for $5 \mathrm{~min}$ and the supernatants were analyzed using reversed-phase HPLC.

In case where ternary complexes were titrated over initiation complexes, experiments were performed essentially the same. For ternary complex Tyr, only EF-Tu.GTP.Tyr-tRNA ${ }^{\text {Tyr }}$ was added at various concentrations $(0.75 / 1 / 2 / 3 \mu \mathrm{M})$ and rapidly mixed with initiation complexes programmed with control mRNA (-/-). For ternary complex Leu, initiation complexes $(0.2 \mu \mathrm{M})$ were rapidly mixed with EF-Tu.GTP.Leu-tRNA ${ }^{\text {Leu }}(0.75 / 1 / 2 / 3 \mu \mathrm{M})$ in the presence of constant amounts of EF-Tu.GTP.Tyr-tRNA ${ }^{\text {Tyr }}(1.5 \mu \mathrm{M})$ and EF-G $(2 \mu \mathrm{M})$. For ternary complex Lys, initiation complexes $(0.2 \mu \mathrm{M})$ were rapidly mixed with EF-Tu.GTP.Lys$\operatorname{tRNA}^{\text {Lys }}(0.75 / 1 / 2 / 3 \mu \mathrm{M})$ in the presence of constant amount of EF-Tu.GTP.aminoacyl-tRNAs for Tyr and Leu $(1.5 \mu \mathrm{M})$ and EF-G $(2 \mu \mathrm{M})$. Reaction products were hydrolyzed with $\mathrm{KOH}$ $(0.8 \mathrm{M})$, neutralized with $1 / 10$ volume glacial acetic acid and analyzed using reversed-phase HPLC. 


\subsubsection{Separation and quantification of the translation products}

Reversed-phase HPLC (LiChroSpher100 RP-8 HPLC column, Merck) was used for the chromatographic analysis of the translation products. Separation of peptides of various sizes (up to pentapeptides) based on their length and hydrophobicity was performed by a linear acetonitrile elution gradient ranging from $0-65 \%$ acetonitrile in $0.1 \%$ TFA (v/v). Each run was $60 \mathrm{~min}$ including column equilibration and $1 \mathrm{ml}$ fractions were collected. Fractions were mixed with Irga-Safe Plus (Perkin Elmer) scintillation liquid $(2 \mathrm{ml})$ and were analyzed by scintillation counting (Tri-Carb 3110 TR, Perkin Elmer). The radioactivity profiles of the chromatograms indicated the elution positions of peptides of various sizes, which were used for the quantification of the respective peptide fraction.

For various ribosomal complexes, incorporation of amino acids was mathematically modeled by using the system of differential equations (1-6). In the system equations $A^{\prime}, B^{\prime}, C^{\prime}, D^{\prime}, E^{\prime}, F^{\prime}$ represent the derivative of the $A, B, C, D, E, F$ which define fraction of $f M, f M Y, f M Y L, f M Y L K$, fMYLKV and fMYLKF peptides present at each time point. The exponential rates $K_{1}(T y r), K_{2}$ (Leu), $\mathrm{K}_{3}$ (Lys), $\mathrm{K}_{4}$ (Val) and $\mathrm{K}_{5}$ (Phe) and the equilibrium concentrations, $\mathrm{Ab}, \mathrm{Bb}, \mathrm{Cb}, \mathrm{Db}$ were fitted by least squares method. The least squares fitting and the numerical integration of the system equations were performed in Scientist software (Micromath).

$$
\begin{aligned}
& A^{\prime}=-K_{1}{ }^{*}(A-A b) \\
& B^{\prime}=K_{1}{ }^{*}(A-A b)-K_{2}{ }^{*}(B-B b) \\
& C^{\prime}=K_{2}{ }^{*}(B-B b)-K_{3}{ }^{*}(C-C b) \\
& D^{\prime}=K_{3}{ }^{*}(C-C b)-\left(K_{4}+K_{5}\right) *(D-D b) \\
& E^{\prime}=K_{4}{ }^{*}(D-D b) \\
& F^{\prime}=K_{5}{ }^{*}(D-D b)
\end{aligned}
$$

\subsubsection{Puromycin assay of $50 \mathrm{~S}$ translocation}

Kinetics of the puromycin reaction was monitored using quench-flow (Figure 19). For monitoring the $50 \mathrm{~S}$ translocation of $\mathrm{POST}_{\text {Lys }}$ complexes, a double mixing experiment was designed. In this case, the quencher to terminate the puromycin reaction needed to be 
applied in a collection tube, which brings a certain delay within the exit line of the quench flow. In order to determine the delay within the exit line we used the classical experimental setup (as explained in section 4.11.3). Initiation complexes (1 pmol) were mixed with puromycin $(1 \mathrm{mM})$ in the reaction loops for different times. Instead of mixing the quencher from the middle drive syringe, reactions were quenched with $\mathrm{KOH}(0.5 \mathrm{M})$ in the collection tube. By single exponential fitting of the fraction of initiation complexes reactive with puromycin over a time using the function $Y=A *\left(1-e^{-k a p p .(x+t)}\right)$, where $x$ stands for the time of reaction in the reaction loop, $t$ represents the delay before quenching, $A$ represent the span and $k_{a p p}$ the rate constant.

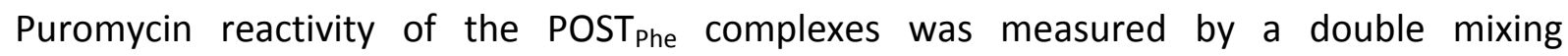
experiment setup. Briefly, the first mixing part took place like in a classical experiment and the equal volumes of initiation complexes $(0.1 \mu \mathrm{M})$ programmed with mRNA (AUG UUC) coding for fMetPhe was rapidly mixed with ternary complex EF-Tu.GTP.Phe tRNA ${ }^{\text {Phe }}(1 \mu \mathrm{M})$ in

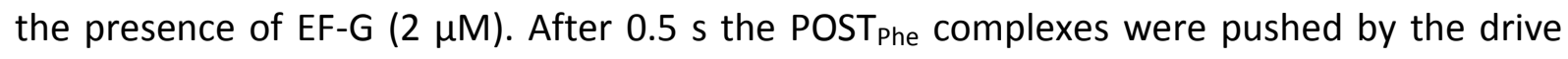
motor through the second mixer where puromycin ( $1 \mathrm{mM})$ was added. The reactants were hold in the exit line for the indicated delay times $0.4,0.9,1.4,2.4,5.4,10.4 \mathrm{~s}$ (0.4 s additional time spent while passing through the exit line was taken in account). The second time the drive motor was fired to push the reactants through the exit line to terminate the puromycin reaction with $\mathrm{KOH}(0.5 \mathrm{M})$ in a collection tube. Alkaline hydrolysis of the reaction products was followed by neutralization and reversed-phase HPLC as stated in section 4.11.4. Fractions of $\left[{ }^{3} \mathrm{H}\right] \mathrm{fMetPhe}-\mathrm{Pmn} /\left[{ }^{3} \mathrm{H}\right]$ total was used for single exponential fitting.

In case where puromycin reactivity of $\mathrm{f}\left[{ }^{3} \mathrm{H}\right]$ MetTyrLeu $\left[{ }^{14} \mathrm{C}\right]$ Lys translation complexes was measured, initiation complex $(0.2 \mu \mathrm{M})$ programmed with frameshifting or control mRNAs were rapidly reacted with ternary complexes EF-Tu.GTP.aa-tRNA (1.5 $\mu \mathrm{M})$ for Tyr, Leu and Lys $(1.5 \mu \mathrm{M})$ and EF-G $(2 \mu \mathrm{M})$ at $37^{\circ} \mathrm{C}$ in buffer A over a time course. In the second mixing step, post-translocation complexes were rapidly mixed with puromycin ( $1 \mathrm{mM}$, final) which was added through the middle drive syringe for $1 \mathrm{~s}$. After passing through the exit line the puromycin reaction was stopped by $\mathrm{KOH}(0.5 \mathrm{M})$. Samples were analyzed by reversed-phase HPLC as described (section 4.11.4). Radioactivity profiles of the chromatograms indicated positions of the $\left[{ }^{3} \mathrm{H}\right]$ fMetTyrLeu $\left[{ }^{14} \mathrm{C}\right]$ Lys and $\left[{ }^{3} \mathrm{H}\right]$ fMetTyrLeu $\left[{ }^{14} \mathrm{C}\right]$ Lys-Pmn peptides at the 
indicated time points. Fractions of $\left[{ }^{3} \mathrm{H}\right] \mathrm{fMetTyrLeu}\left[{ }^{14} \mathrm{C}\right]$ Lys-Pmn $/\left[{ }^{3} \mathrm{H}\right]$ total were evaluated by single exponential fitting using GraphPad Prism.

\subsubsection{Kinetics of translocation measured by stopped-flow}

Fluorescence stopped-flow measurements were performed using a SX-20MV stopped-flow apparatus (Applied Photophysics, Leatherhead, UK). To measure translocation of tRNA ${ }^{\text {Lys }}$ from the $A$ to the $P$ site, proflavinated-tRNA ${ }^{\text {Lys }}$ was used (Rodnina et al., 1994; Wilden et al., 2006; Wintermeyer and Zachau, 1979). Fluorescence of proflavin was excited at $470 \mathrm{~nm}$ and emission was monitored after passing a KV500 cut-off filter (Schott). Experiments were performed in buffer $A$ with DTT $(1 \mathrm{mM})$ at $37^{\circ} \mathrm{C}$ by rapidly mixing equal volumes $(60 \mu \mathrm{l})$ of initiation complexes $(0.2 \mu \mathrm{M})$ with ternary complexes, EF-Tu.GTP.aa-tRNA (1.5 $\mu \mathrm{M}$ each) for Tyr, Leu, Lys, Val and Phe and EF-G $(2 \mu \mathrm{M})$. Fluorescence change was measured over a time course of 100 seconds and 1000 points were acquired in logarithmic sampling mode. Data were evaluated by three exponential fitting using the function, $F=F \infty+A . e^{-k a p p 1 . t}+B . e^{-}$ kapp2.t $+C \cdot e^{-k a p p 3 \cdot t}$ in TableCurve software (Jandel Scientific), where $F$ is the fluorescence signal at time $t, F \infty$ is the final fluorescence, $k_{a p p 1}, k_{a p p 2}$ and $k_{a p p 3}$ are apparent rate constants and $A, B$ and $C$ are the change in amplitudes.

\subsubsection{Kinetics of EF-G binding measured using stopped flow}

For EF-G binding experiments post tRNA ${ }^{\text {Leu }}$ translocation complexes were formed by mixing $80 \mu \mathrm{l}$ each of $70 \mathrm{~S}$ initiation complexes (L7/12 138Alx488) $(0.5 \mu \mathrm{M})$ programmed with mRNAs encoding for MetTyrLeuLys with ternary complexes EF-Tu.GTP-aa-tRNA for Tyr and Leu (1.5-1 $\mu \mathrm{M}$, respectively) and EF-G $(2 \mu \mathrm{M})$ for 30 seconds at $37^{\circ} \mathrm{C}$. Post-translocation complexes $\left(0.05 \mu \mathrm{M}\right.$, final) and were rapidly mixed with ternary complex, EF-Tu.GTP.Lys-tRNA ${ }^{\text {Lys }}(0.25$ $\mu \mathrm{M}$, final) and EF-G (209QSY9) $\left(1 \mu \mathrm{M}\right.$, final) at $37^{\circ} \mathrm{C}$ in buffer A with DTT (1 mM) in a stoppedflow apparatus (Applied Photophysics). Excitation wavelength was set to $470 \mathrm{~nm}$ and fluorescence emission was recorded after passing through a KV500 cut-off filter (Schott). 1000 points were acquired over 10-20 seconds on a logarithmic time scale. Data were evaluated by fitting to three-exponential functions as described in section 4.11.6. 


\section{BIBLIOGRAPHY}

Adamski, F.M., Donly, B.C., and Tate, W.P. (1993). Competition between frameshifting, termination and suppression at the frameshift site in the Escherichia coli release factor-2 mRNA. Nucleic Acids Res 21, 5074-5078.

Agrawal, R., Heagle, A., Penczek, P., Grassucci, R., and Frank, J. (1999). EF-G-dependent GTP hydrolysis induces translocation accompanied by large conformational changes in the 705 ribosome. Nature structural biology 6, 643-647.

Agrawal, R., Penczek, P., Grassucci, R., and Frank, J. (1998). Visualization of elongation factor G on the Escherichia coli 70S ribosome: the mechanism of translocation. Proceedings of the National Academy of Sciences of the United States of America 95, 6134-6138.

Agrawal, R.K., Spahn, C.M., Penczek, P., Grassucci, R.A., Nierhaus, K.H., and Frank, J. (2000). Visualization of tRNA movements on the Escherichia coli $70 \mathrm{~S}$ ribosome during the elongation cycle. The Journal of cell biology 150, 447-460.

Agris, P.F. (2004). Decoding the genome: a modified view. Nucleic Acids Res 32, 223-238.

Agris, P.F. (2008). Bringing order to translation: the contributions of transfer RNA anticodon-domain modifications. EMBO reports 9, 629-635.

Asakura, T., Sasaki, T., Nagano, F., Satoh, A., Obaishi, H., Nishioka, H., Imamura, H., Hotta, K., Tanaka, K., Nakanishi, H., et al. (1998). Isolation and characterization of a novel actin filament-binding protein from Saccharomyces cerevisiae. Oncogene 16, 121-130.

Atkins, J., Baranov, P., Fayet, O., Herr, A., Howard, M., Ivanov, I., Matsufuji, S., Miller, W., Moore, B., Prère, M., et al. (2001). Overriding standard decoding: implications of recoding for ribosome function and enrichment of gene expression. Cold Spring Harbor symposia on quantitative biology 66, 217232.

Atkins, J.F., and Gesteland, R.F. (2009). Recoding: expansion of decoding rules enriches gene expression. Springer 24 . 
Barak, Z., Lindsley, D., and Gallant, J. (1996). On the mechanism of leftward frameshifting at several hungry codons. Journal of molecular biology 256, 676-684.

Baranov, P., Gesteland, R., and Atkins, J. (2002a). Recoding: translational bifurcations in gene expression. Gene 286, 187-201.

Baranov, P., Gesteland, R., and Atkins, J. (2004). P-site tRNA is a crucial initiator of ribosomal frameshifting. RNA (New York, NY) 10, 221-251.

Baranov, P.V., Gesteland, R.F., and Atkins, J.F. (2002b). Release factor 2 frameshifting sites in different bacteria. EMBO reports 3, 373-377.

Bashan, A., and Yonath, A. (2008). Correlating ribosome function with high-resolution structures. Trends in microbiology 16, 326-335.

Bekaert, M., and Rousset, J.-P. (2005). An extended signal involved in eukaryotic -1 frameshifting operates through modification of the E site tRNA. Molecular cell 17, 61-68.

Belcourt, M.F., and Farabaugh, P.J. (1990). Ribosomal frameshifting in the yeast retrotransposon Ty: tRNAs induce slippage on a 7 nucleotide minimal site. Cell 62, 339-352.

Bertrand, C., Prere, M.F., Gesteland, R.F., Atkins, J.F., and Fayet, O. (2002). Influence of the stacking potential of the base $3^{\prime}$ of tandem shift codons on -1 ribosomal frameshifting used for gene expression. Rna 8, 16-28.

Biswas, P., Jiang, X., Pacchia, A., Dougherty, J., and Peltz, S. (2004). The human immunodeficiency virus type 1 ribosomal frameshifting site is an invariant sequence determinant and an important target for antiviral therapy. Journal of virology 78, 2082-2089.

Björk, G.R. (1995). Genetic Dissection of Synthesis and Function of Modified Nucleosides in Bacterial Transfer RNA. In Progress in Nucleic Acid Research and Molecular Biology, E.C. Waldo, and M. Kivie, eds. (Academic Press), pp. 263-338.

Blanchard, S., Kim, H., Gonzalez, R., Puglisi, J., and Chu, S. (2004). tRNA dynamics on the ribosome during translation. Proceedings of the National Academy of Sciences of the United States of America $101,12893-12898$. 
Blattner, F.R., Plunkett, G., 3rd, Bloch, C.A., Perna, N.T., Burland, V., Riley, M., Collado-Vides, J., Glasner, J.D., Rode, C.K., Mayhew, G.F., et al. (1997). The complete genome sequence of Escherichia coli K-12. Science 277, 1453-1462.

Blinkowa, A.L., and Walker, J.R. (1990). Programmed ribosomal frameshifting generates the Escherichia coli DNA polymerase III gamma subunit from within the tau subunit reading frame. Nucleic Acids Res 18, 1725-1729.

Bock, A., Forchhammer, K., Heider, J., and Baron, C. (1991). Selenoprotein synthesis: an expansion of the genetic code. Trends Biochem Sci 16, 463-467.

Bonetti, B., Fu, L., Moon, J., and Bedwell, D.M. (1995). The efficiency of translation termination is determined by a synergistic interplay between upstream and downstream sequences in Saccharomyces cerevisiae. J Mol Biol 251, 334-345.

Borowski, C., Rodnina, M.V., and Wintermeyer, W. (1996). Truncated elongation factor G lacking the $\mathrm{G}$ domain promotes translocation of the $3^{\prime}$ end but not of the anticodon domain of peptidyl-tRNA. Proc Natl Acad Sci U S A 93, 4202-4206.

Brault, V., and Miller, W.A. (1992). Translational frameshifting mediated by a viral sequence in plant cells. Proc Natl Acad Sci U S A 89, 2262-2266.

Brierley, I., Digard, P., and Inglis, S. (1989). Characterization of an efficient coronavirus ribosomal frameshifting signal: requirement for an RNA pseudoknot. Cell 57, 537-547.

Brierley, I., Gilbert, R.C., and Pennell, S. (2010). Pseudoknot-Dependent Programmed -1 Ribosomal Frameshifting: Structures, Mechanisms and Models. In Recoding: Expansion of Decoding Rules Enriches Gene Expression, J.F. Atkins, and R.F. Gesteland, eds. (Springer New York), pp. 149-174.

Brierley, I., Jenner, A., and Inglis, S. (1992). Mutational analysis of the "slippery-sequence" component of a coronavirus ribosomal frameshifting signal. Journal of molecular biology $227,463-$ 479.

Brierley, I., Meredith, M., Bloys, A., and Hagervall, T. (1997). Expression of a coronavirus ribosomal frameshift signal in Escherichia coli: influence of tRNA anticodon modification on frameshifting. Journal of molecular biology 270, 360-373. 
Brierley, I., Rolley, N.J., Jenner, A.J., and Inglis, S.C. (1991). Mutational analysis of the RNA pseudoknot component of a coronavirus ribosomal frameshifting signal. J Mol Biol 220, 889-902.

Brunelle, J.L., Shaw, J.J., Youngman, E.M., and Green, R. (2008). Peptide release on the ribosome depends critically on the $2^{\prime} \mathrm{OH}$ of the peptidyl-tRNA substrate. Rna 14, 1526-1531.

Buchan, J.R., and Stansfield, I. (2007). Halting a cellular production line: responses to ribosomal pausing during translation. Biol Cell 99, 475-487.

Byrne, R.T., Konevega, A.L., Rodnina, M.V., and Antson, A.A. (2010). The crystal structure of unmodified tRNAPhe from Escherichia coli. Nucleic Acids Res 38, 4154-4162.

Clark, M.B., Janicke, M., Gottesbuhren, U., Kleffmann, T., Legge, M., Poole, E.S., and Tate, W.P. (2007). Mammalian gene PEG10 expresses two reading frames by high efficiency -1 frameshifting in embryonic-associated tissues. J Biol Chem 282, 37359-37369.

Commans, S., and Böck, A. (1999). Selenocysteine inserting tRNAs: an overview. FEMS microbiology reviews $23,335-351$.

Craigen, W.J., and Caskey, C.T. (1986). Expression of peptide chain release factor 2 requires highefficiency frameshift. Nature 322, 273-275.

Crick, F. (1966). Codon--anticodon pairing: the wobble hypothesis. Journal of molecular biology 19, 548-555.

Cunha, C.E.B., R.; Peske, F.; Holtkamp, W.; Wintermeyer, W.; Rodnina M.V. (2012). Dual use of GTP hydrolysis by elongation factor $\mathrm{G}$ on the ribosome. Translation.

Curran, J.F. (1993). Analysis of effects of tRNA:message stability on frameshift frequency at the Escherichia coli RF2 programmed frameshift site. Nucleic Acids Res 21, 1837-1843.

Danilevskaya, O., Slot, F., Pavlova, M., and Pardue, M.L. (1994). Structure of the Drosophila HeT-A transposon: a retrotransposon-like element forming telomeres. Chromosoma 103, 215-224.

Daviter, T., Gromadski, K.B., and Rodnina, M.V. (2006). The ribosome's response to codon-anticodon mismatches. Biochimie 88, 1001-1011. 
Devaraj, A., and Fredrick, K. (2010). Short spacing between the Shine-Dalgarno sequence and P codon destabilizes codon-anticodon pairing in the $\mathrm{P}$ site to promote +1 programmed frameshifting. Mol Microbiol 78, 1500-1509.

Di, R., Dinesh-Kumar, S.P., and Miller, W.A. (1993). Translational frameshifting by barley yellow dwarf virus RNA (PAV serotype) in Escherichia coli and in eukaryotic cell-free extracts. Molecular plantmicrobe interactions : MPMI 6, 444-452.

Diaconu, M., Kothe, U., Schlunzen, F., Fischer, N., Harms, J.M., Tonevitsky, A.G., Stark, H., Rodnina, M.V., and Wahl, M.C. (2005). Structural basis for the function of the ribosomal L7/12 stalk in factor binding and GTPase activation. Cell 121, 991-1004.

Dinman, J. (2012). Mechanisms and implications of programmed translational frameshifting. Wiley interdisciplinary reviews RNA 3, 661-673.

Dinman, J., Ruiz-Echevarria, M., Czaplinski, K., and Peltz, S. (1997). Peptidyl-transferase inhibitors have antiviral properties by altering programmed -1 ribosomal frameshifting efficiencies: development of model systems. Proceedings of the National Academy of Sciences of the United States of America 94, 6606-6611.

Doerfel, L.K., Wohlgemuth, I., Kothe, C., Peske, F., Urlaub, H., and Rodnina, M.V. (2013). EF-P is essential for rapid synthesis of proteins containing consecutive proline residues. Science 339, 85-88.

Donly, B., Edgar, C., Adamski, F., and Tate, W. (1990). Frameshift autoregulation in the gene for Escherichia coli release factor 2: partly functional mutants result in frameshift enhancement. Nucleic acids research $18,6517-6522$.

Dorner, S., Brunelle, J.L., Sharma, D., and Green, R. (2006). The hybrid state of tRNA binding is an authentic translation elongation intermediate. Nat Struct Mol Biol 13, 234-241.

Dulude, D., Theberge-Julien, G., Brakier-Gingras, L., and Heveker, N. (2008). Selection of peptides interfering with a ribosomal frameshift in the human immunodeficiency virus type 1. Rna 14, 981991. 
Ermolenko, D.N., Spiegel, P.C., Majumdar, Z.K., Hickerson, R.P., Clegg, R.M., and Noller, H.F. (2007). The antibiotic viomycin traps the ribosome in an intermediate state of translocation. Nat Struct Mol Biol 14, 493-497.

Farabaugh, P. (1996a). Programmed translational frameshifting. Microbiological reviews 60, 103-134.

Farabaugh, P.J. (1996b). Programmed translational frameshifting. Annual review of genetics 30, 507528.

Farabaugh, P.J. (2010). Programmed frameshifting in budding yeast. Springer, 221-247.

Fayet, O., and Prère, M.-F. (2010). Programmed Ribosomal -1 Frameshifting as a Tradition: The Bacterial Transposable Elements of the IS3 Family. In Recoding: Expansion of Decoding Rules Enriches Gene Expression, J.F. Atkins, and R.F. Gesteland, eds. (Springer New York), pp. 259-280.

Feng, Y.X., Yuan, H., Rein, A., and Levin, J.G. (1992). Bipartite signal for read-through suppression in murine leukemia virus mRNA: an eight-nucleotide purine-rich sequence immediately downstream of the gag termination codon followed by an RNA pseudoknot. J Virol 66, 5127-5132.

Fischer, N., Konevega, A.L., Wintermeyer, W., Rodnina, M.V., and Stark, H. (2010). Ribosome dynamics and tRNA movement by time-resolved electron cryomicroscopy. Nature 466, 329-333.

Flower, A., and McHenry, C. (1990). The gamma subunit of DNA polymerase III holoenzyme of Escherichia coli is produced by ribosomal frameshifting. Proceedings of the National Academy of Sciences of the United States of America 87, 3713-3717.

Fredrick, K., and Noller, H. (2003). Catalysis of ribosomal translocation by sparsomycin. Science (New York, NY) 300, 1159-1162.

Gao, Y.G., Selmer, M., Dunham, C.M., Weixlbaumer, A., Kelley, A.C., and Ramakrishnan, V. (2009). The structure of the ribosome with elongation factor $\mathrm{G}$ trapped in the posttranslocational state. Science 326, 694-699.

Garcia, A., van Duin, J., and Pleij, C.W. (1993). Differential response to frameshift signals in eukaryotic and prokaryotic translational systems. Nucleic Acids Res 21, 401-406. 
Gesteland, R., and Atkins, J. (1996). Recoding: dynamic reprogramming of translation. Annual review of biochemistry $65,741-809$.

Giedroc, D., and Cornish, P. (2009). Frameshifting RNA pseudoknots: structure and mechanism. Virus research 139, 193-401.

Gold, L. (1988). Posttranscriptional regulatory mechanisms in Escherichia coli. Annu Rev Biochem 57, 199-233.

Green, L., Kim, C.H., Bustamante, C., and Tinoco, I., Jr. (2008). Characterization of the mechanical unfolding of RNA pseudoknots. J Mol Biol 375, 511-528.

Grentzmann, G., Ingram, J., Kelly, P., Gesteland, R., and Atkins, J. (1998a). A dual-luciferase reporter system for studying recoding signals. RNA (New York, NY) 4, 479-565.

Grentzmann, G., Kelly, P., Laalami, S., Shuda, M., Firpo, M., Cenatiempo, Y., and Kaji, A. (1998b). Release factor RF-3 GTPase activity acts in disassembly of the ribosome termination complex. RNA (New York, NY) 4, 973-983.

Grigoriadou, C., Marzi, S., Kirillov, S., Gualerzi, C., and Cooperman, B. (2007). A quantitative kinetic scheme for $70 \mathrm{~S}$ translation initiation complex formation. Journal of molecular biology 373, 562-572.

Gromadski, K.B., Daviter, T., and Rodnina, M.V. (2006). A uniform response to mismatches in codonanticodon complexes ensures ribosomal fidelity. Mol Cell 21, 369-377.

Gromadski, K.B., and Rodnina, M.V. (2004). Kinetic determinants of high-fidelity tRNA discrimination on the ribosome. Mol Cell 13, 191-200.

Gromadski, K.B., Wieden, H.J., and Rodnina, M.V. (2002). Kinetic mechanism of elongation factor Tscatalyzed nucleotide exchange in elongation factor Tu. Biochemistry 41, 162-169.

Gualerzi, C., Brandi, L., Caserta, E., Garofalo, C., Lammi, M., La Teana, A., Petrelli, D., Spurio, R., Tomsic, J., and Pon, C. (2001). Initiation factors in the early events of mRNA translation in bacteria. Cold Spring Harbor symposia on quantitative biology 66, 363-376. 
Gualerzi, C., and Pon, C. (1990). Initiation of mRNA translation in prokaryotes. Biochemistry 29, 58815889.

Guo, D., Mant, C., Taneja, A., and Hodges, R. (1986a). Prediction of peptide retention times in reversed-phase high-performance liquid chromatography II. Correlation of observed and predicted peptide retention times .... Journal of Chromatography A.

Guo, D., Mant, C., Taneja, A., and Parker..., J. (1986b). Prediction of peptide retention times in reversed-phase high-performance liquid chromatography I. Determination of retention coefficients of amino acid residues of ....... of Chromatography $A$.

Guo, Z., and Noller, H.F. (2012). Rotation of the head of the 30 S ribosomal subunit during mRNA translocation. Proc Natl Acad Sci U S A.

Gurvich, O., Baranov, P., Gesteland, R., and Atkins, J. (2005). Expression levels influence ribosomal frameshifting at the tandem rare arginine codons AGG_AGG and AGA_AGA in Escherichia coli. Journal of bacteriology 187, 4023-4032.

Hammell, A., Taylor, R., Peltz, S., and Dinman, J. (1999). Identification of putative programmed -1 ribosomal frameshift signals in large DNA databases. Genome research 9, 417-427.

Hansen, T.M., Reihani, S.N., Oddershede, L.B., and Sorensen, M.A. (2007). Correlation between mechanical strength of messenger RNA pseudoknots and ribosomal frameshifting. Proc Natl Acad Sci U S A $104,5830-5835$.

Harger, J., Meskauskas, A., and Dinman, J. (2002). An "integrated model" of programmed ribosomal frameshifting. Trends in biochemical sciences 27, 448-502.

Hartz, D., Binkley, J., Hollingsworth, T., and Gold, L. (1990). Domains of initiator tRNA and initiation codon crucial for initiator tRNA selection by Escherichia coli IF3. Genes \& development 4, 1790-1800.

Hartz, D., Mcpheeters, D.S., and Gold, L. (1989). Selection of the Initiator Transfer-Rna by EscherichiaColi Initiation-Factors. Genes \& Development 3, 1899-1912.

Herr, A., Atkins, J., and Gesteland, R. (2000). Coupling of open reading frames by translational bypassing. Annual review of biochemistry 69, 343-372. 
Herr, A., Nelson, C., Wills, N., Gesteland, R., and Atkins, J. (2001a). Analysis of the roles of tRNA structure, ribosomal protein L9, and the bacteriophage T4 gene 60 bypassing signals during ribosome slippage on mRNA. Journal of molecular biology 309, 1029-1048.

Herr, A., Wills, N., Nelson, C., Gesteland, R., and Atkins, J. (2001b). Drop-off during ribosome hopping. Journal of molecular biology 311, 445-452.

Horsfield, J., Wilson, D., Mannering, S., Adamski, F., and Tate, W. (1995). Prokaryotic ribosomes recode the HIV-1 gag-pol-1 frameshift sequence by an E/P site post-translocation simultaneous slippage mechanism. Nucleic acids research 23, 1487-1494.

Howard, M.T., Gesteland, R.F., and Atkins, J.F. (2004). Efficient stimulation of site-specific ribosome frameshifting by antisense oligonucleotides. Rna 10, 1653-1661.

Huang, W.M., Ao, S.Z., Casjens, S., Orlandi, R., Zeikus, R., Weiss, R., Winge, D., and Fang, M. (1988). A persistent untranslated sequence within bacteriophage T4 DNA topoisomerase gene 60. Science 239, 1005-1012.

Irvine, J.H., Horsfield, J.A., McKinney, C.Z., and Tate, W.P. (1998). A novel strategy to interfere with human immunodeficiency virus type 1 propagation. The New Zealand medical journal 111, 222-224.

Ito, K., Uno, M., and Nakamura, Y. (2000). A tripeptide 'anticodon' deciphers stop codons in messenger RNA. Nature 403, 680-684.

Jacks, T., Madhani, H., Masiarz, F., and Varmus, H. (1988a). Signals for ribosomal frameshifting in the Rous sarcoma virus gag-pol region. Cell 55, 447-505.

Jacks, T., Power, M.D., Masiarz, F.R., Luciw, P.A., Barr, P.J., and Varmus, H.E. (1988b). Characterization of ribosomal frameshifting in HIV-1 gag-pol expression. Nature 331, 280-283.

Jacobs, J.L., Belew, A.T., Rakauskaite, R., and Dinman, J.D. (2007). Identification of functional, endogenous programmed- 1 ribosomal frameshift signals in the genome of Saccharomyces cerevisiae. Nucleic acids research $35,165-174$.

Jenner, L.B., Demeshkina, N., Yusupova, G., and Yusupov, M. (2010). Structural aspects of messenger RNA reading frame maintenance by the ribosome. Nat Struct Mol Biol 17, 555-560. 
Jones, O.W., Jr., and Nirenberg, M.W. (1966). Degeneracy in the amino acid code. Biochimica et biophysica acta 119, 400-406.

Juhling, F., Morl, M., Hartmann, R.K., Sprinzl, M., Stadler, P.F., and Putz, J. (2009). tRNAdb 2009: compilation of tRNA sequences and tRNA genes. Nucleic Acids Res 37, D159-162.

Julian, P., Konevega, A.L., Scheres, S.H., Lazaro, M., Gil, D., Wintermeyer, W., Rodnina, M.V., and Valle, M. (2008). Structure of ratcheted ribosomes with tRNAs in hybrid states. Proc Natl Acad Sci U S A $105,16924-16927$.

Julian, P., Milon, P., Agirrezabala, X., Lasso, G., Gil, D., Rodnina, M.V., and Valle, M. (2011). The CryoEM structure of a complete $30 \mathrm{~S}$ translation initiation complex from Escherichia coli. PLoS biology 9, e1001095.

Katunin, V., Muth, G., Strobel, S., Wintermeyer, W., and Rodnina, M. (2002). Important contribution to catalysis of peptide bond formation by a single ionizing group within the ribosome. Molecular cell 10, 339-346.

Klagges, B.R., Heimbeck, G., Godenschwege, T.A., Hofbauer, A., Pflugfelder, G.O., Reifegerste, R., Reisch, D., Schaupp, M., Buchner, S., and Buchner, E. (1996). Invertebrate synapsins: a single gene codes for several isoforms in Drosophila. The Journal of neuroscience : the official journal of the Society for Neuroscience 16, 3154-3165.

Kolor, K., Lindsley, D., and Gallant, J.A. (1993). On the role of the P-site in leftward ribosome frameshifting at a hungry codon. J Mol Biol 230, 1-5.

Kontos, H., Napthine, S., and Brierley, I. (2001). Ribosomal pausing at a frameshifter RNA pseudoknot is sensitive to reading phase but shows little correlation with frameshift efficiency. Mol Cell Biol 21, 8657-8670.

Kothe, U., Wieden, H.J., Mohr, D., and Rodnina, M.V. (2004). Interaction of helix D of elongation factor Tu with helices 4 and 5 of protein L7/12 on the ribosome. J Mol Biol 336, 1011-1021.

Kramer, E.B., and Farabaugh, P.J. (2007). The frequency of translational misreading errors in E. coli is largely determined by tRNA competition. Rna 13, 87-96. 
Krüger, M., Pedersen, S., Hagervall, T., and Sørensen, M. (1998). The modification of the wobble base of tRNAGlu modulates the translation rate of glutamic acid codons in vivo. Journal of molecular biology 284, 621-631.

Kubarenko, A., Sergiev, P., Wintermeyer, W., Dontsova, O., and Rodnina, M. (2006). Involvement of helix 34 of $16 \mathrm{~S}$ rRNA in decoding and translocation on the ribosome. The Journal of biological chemistry $281,35235-35244$.

Kuhlenkoetter, S., Wintermeyer, W., and Rodnina, M.V. (2011). Different substrate-dependent transition states in the active site of the ribosome. Nature 476, 351-354.

Larsen, B., Gesteland, R.F., and Atkins, J.F. (1997). Structural probing and mutagenic analysis of the stem-loop required for Escherichia coli dnaX ribosomal frameshifting: programmed efficiency of $50 \%$. J Mol Biol 271, 47-60.

Larsen, B., Wills, N.M., Gesteland, R.F., and Atkins, J.F. (1994). rRNA-mRNA base pairing stimulates a programmed -1 ribosomal frameshift. Journal of bacteriology 176, 6842-6851.

Leger, M., Dulude, D., Steinberg, S.V., and Brakier-Gingras, L. (2007). The three transfer RNAs occupying the $A, P$ and $E$ sites on the ribosome are involved in viral programmed -1 ribosomal frameshift. Nucleic Acids Res 35, 5581-5592.

Li, G., and Rice, C. (1993). The signal for translational readthrough of a UGA codon in Sindbis virus RNA involves a single cytidine residue immediately downstream of the termination codon. Journal of virology 67, 5062-5067.

Licznar, P., Mejlhede, N., Prère, M.-F., Wills, N., Gesteland, R., Atkins, J., and Fayet, O. (2003). Programmed translational -1 frameshifting on hexanucleotide motifs and the wobble properties of tRNAs. The EMBO journal 22, 4770-4778.

Lopinski, J., Dinman, J., and Bruenn, J. (2000). Kinetics of ribosomal pausing during programmed -1 translational frameshifting. Molecular and cellular biology 20, 1095-1103.

Manktelow, E., Shigemoto, K., and Brierley, I. (2005). Characterization of the frameshift signal of Edr, a mammalian example of programmed -1 ribosomal frameshifting. Nucleic Acids Res 33, 1553-1563. 
Marcheschi, R.J., Staple, D.W., and Butcher, S.E. (2007). Programmed ribosomal frameshifting in SIV is induced by a highly structured RNA stem-loop. J Mol Biol 373, 652-663.

Marczinke, B., Bloys, A.J., Brown, T.D., Willcocks, M.M., Carter, M.J., and Brierley, I. (1994). The human astrovirus RNA-dependent RNA polymerase coding region is expressed by ribosomal frameshifting. J Virol 68, 5588-5595.

Marquez, V., Wilson, D.N., Tate, W.P., Triana-Alonso, F., and Nierhaus, K.H. (2004). Maintaining the ribosomal reading frame: the influence of the E site during translational regulation of release factor 2. Cell $118,45-55$.

Matsufuji, S., Matsufuji, T., Miyazaki, Y., Murakami, Y., Atkins, J.F., Gesteland, R.F., and Hayashi, S. (1995). Autoregulatory frameshifting in decoding mammalian ornithine decarboxylase antizyme. Cell $80,51-60$.

Mazauric, M.H., Leroy, J.L., Visscher, K., Yoshizawa, S., and Fourmy, D. (2009). Footprinting analysis of BWYV pseudoknot-ribosome complexes. Rna 15, 1775-1786.

Mejlhede, N., Atkins, J.F., and Neuhard, J. (1999). Ribosomal -1 frameshifting during decoding of Bacillus subtilis cdd occurs at the sequence CGA AAG. J Bacteriol 181, 2930-2937.

Meskauskas, A., Harger, J., Jacobs, K., and Dinman, J. (2003). Decreased peptidyltransferase activity correlates with increased programmed -1 ribosomal frameshifting and viral maintenance defects in the yeast Saccharomyces cerevisiae. RNA (New York, NY) 9, 982-992.

Meulenberg, J.J., Hulst, M.M., de Meijer, E.J., Moonen, P.L., den Besten, A., de Kluyver, E.P., Wensvoort, G., and Moormann, R.J. (1993). Lelystad virus, the causative agent of porcine epidemic abortion and respiratory syndrome (PEARS), is related to LDV and EAV. Virology 192, 62-72.

Milon, P., Carotti, M., Konevega, A.L., Wintermeyer, W., Rodnina, M.V., and Gualerzi, C.O. (2010). The ribosome-bound initiation factor 2 recruits initiator tRNA to the $30 \mathrm{~S}$ initiation complex. EMBO reports $11,312-316$.

Milon, P., Konevega, A., Gualerzi, C., and Rodnina, M. (2008). Kinetic checkpoint at a late step in translation initiation. Molecular cell 30, 712-720. 
Milon, P., Konevega, A., Peske, F., Fabbretti, A., Gualerzi, C., and Rodnina, M. (2007). Transient kinetics, fluorescence, and FRET in studies of initiation of translation in bacteria. Methods in enzymology 430, 1-30.

Milon, P., Maracci, C., Filonava, L., Gualerzi, C.O., and Rodnina, M.V. (2012). Real-time assembly landscape of bacterial 30 S translation initiation complex. Nat Struct Mol Biol 19, 609-615.

Moazed, D., and Noller, H.F. (1989). Intermediate states in the movement of transfer RNA in the ribosome. Nature 342, 142-148.

Modolell, J., and Vazquez (1977). The inhibition of ribosomal translocation by viomycin. European journal of biochemistry / FEBS 81, 491-497.

Mohr, D., Wintermeyer, W., and Rodnina, M.V. (2002). GTPase activation of elongation factors Tu and $\mathrm{G}$ on the ribosome. Biochemistry 41, 12520-12528.

Moon, S., Byun, Y., Kim, H.-J., Jeong, S., and Han, K. (2004). Predicting genes expressed via -1 and +1 frameshifts. Nucleic acids research 32, 4884-4892.

Morikawa, S., and Bishop, D.H. (1992). Identification and analysis of the gag-pol ribosomal frameshift site of feline immunodeficiency virus. Virology 186, 389-397.

Morris, D.K., and Lundblad, V. (1997). Programmed translational frameshifting in a gene required for yeast telomere replication. Current biology : CB 7, 969-976.

Munro, J., Altman, R., O'Connor, N., and Blanchard, S. (2007). Identification of two distinct hybrid state intermediates on the ribosome. Molecular cell 25, 505-517.

Munro, J.B., Wasserman, M.R., Altman, R.B., Wang, L., and Blanchard, S.C. (2010). Correlated conformational events in EF-G and the ribosome regulate translocation. Nat Struct Mol Biol 17, 14701477.

Murphy, F.V.t., Ramakrishnan, V., Malkiewicz, A., and Agris, P.F. (2004). The role of modifications in codon discrimination by tRNA(Lys)UUU. Nat Struct Mol Biol 11, 1186-1191. 
Namy, O., Moran, S.J., Stuart, D.I., Gilbert, R.J., and Brierley, I. (2006). A mechanical explanation of RNA pseudoknot function in programmed ribosomal frameshifting. Nature 441, 244-247.

Namy, O., and Rousset, J.-P. (2010). Specification of standard amino acids by stop codons. Springer, 79-100.

Namy, O., Rousset, J., and Napthine, S. (2004). Reprogrammed genetic decoding in cellular gene expression. Molecular cell.

Napthine, S., Liphardt, J., Bloys, A., Routledge, S., and Brierley, I. (1999). The role of RNA pseudoknot stem 1 length in the promotion of efficient -1 ribosomal frameshifting. Journal of molecular biology $288,305-325$.

Napthine, S., Vidakovic, M., Girnary, R., Namy, O., and Brierley, I. (2003). Prokaryotic-style frameshifting in a plant translation system: conservation of an unusual single-tRNA slippage event. EMBO J 22, 3941-3950.

Nguyen, B., Ticu, C., and Wilson, K.S. (2010). Intramolecular movements in EF-G, trapped at different stages in its GTP hydrolytic cycle, probed by FRET. J Mol Biol 397, 1245-1260.

Nierhaus, K. (2006). Decoding errors and the involvement of the E-site. Biochimie 88, 1013-1019.

Nikolic, E.I., King, L.M., Vidakovic, M., Irigoyen, N., and Brierley, I. (2012). Modulation of ribosomal frameshifting frequency and its effect on the replication of Rous sarcoma virus. J Virol 86, 1158111594.

Noller, H., Yusupov, M., Yusupova, G., Baucom, A., and Cate, J. (2002). Translocation of tRNA during protein synthesis. FEBS letters 514, 11-16.

Noller, H.F., Hoffarth, V., and Zimniak, L. (1992). Unusual resistance of peptidyl transferase to protein extraction procedures. Science 256, 1416-1419.

Ortiz-Meoz, R., and Green, R. (2010). Functional elucidation of a key contact between tRNA and the large ribosomal subunit rRNA during decoding. RNA (New York, NY) 16, 2002-2013. 
Ortiz, P., Ulloque, R., Kihara, G., Zheng, H., and Kinzy, T. (2006). Translation elongation factor 2 anticodon mimicry domain mutants affect fidelity and diphtheria toxin resistance. The Journal of biological chemistry 281, 32639-32648.

Osterman, I.A., Evfratov, S.A., Sergiev, P.V., and Dontsova, O.A. (2013). Comparison of mRNA features affecting translation initiation and reinitiation. Nucleic Acids Research 41, 474-486.

Paleskava, A., Konevega, A., and Rodnina, M. (2010). Thermodynamic and kinetic framework of selenocysteyl-tRNASec recognition by elongation factor SelB. The Journal of biological chemistry 285 , 3014-3020.

Pan, D., Kirillov, S., and Cooperman, B. (2007). Kinetically competent intermediates in the translocation step of protein synthesis. Molecular cell 25, 519-548.

Pape, T., Wintermeyer, W., and Rodnina, M. (1998). Complete kinetic mechanism of elongation factor Tu-dependent binding of aminoacyl-tRNA to the A site of the E. coli ribosome. The EMBO journal $17,7490-7497$.

Parker, J. (1989). Errors and alternatives in reading the universal genetic code. Microbiol Rev 53, $273-$ 298.

Peltz, S., Hammell, A., Cui, Y., Yasenchak, J., Puljanowski, L., and Dinman, J. (1999). Ribosomal protein L3 mutants alter translational fidelity and promote rapid loss of the yeast killer virus. Molecular and cellular biology 19, 384-391.

Peske, F., Rodnina, M.V., and Wintermeyer, W. (2005). Sequence of steps in ribosome recycling as defined by kinetic analysis. Mol Cell 18, 403-412.

Peske, F., Savelsbergh, A., Katunin, V.I., Rodnina, M.V., and Wintermeyer, W. (2004). Conformational changes of the small ribosomal subunit during elongation factor G-dependent tRNA-mRNA translocation. J Mol Biol 343, 1183-1194.

Petropoulos, A., and Green, R. (2012). Further in vitro exploration fails to support the allosteric threesite model. The Journal of biological chemistry $287,11642-11648$. 
Phelps, S.S., Malkiewicz, A., Agris, P.F., and Joseph, S. (2004). Modified nucleotides in tRNA(Lys) and tRNA(Val) are important for translocation. J Mol Biol 338, 439-444.

Plant, E., Rakauskaite, R., Taylor, D., and Dinman, J. (2010). Achieving a golden mean: mechanisms by which coronaviruses ensure synthesis of the correct stoichiometric ratios of viral proteins. Journal of virology $84,4330-4340$.

Plant, E.P. (2003). The 9-A solution: How mRNA pseudoknots promote efficient programmed -1 ribosomal frameshifting. Rna 9, 168-174.

Plant, E.P., and Dinman, J.D. (2005). Torsional restraint: a new twist on frameshifting pseudoknots. Nucleic Acids Res 33, 1825-1833.

Prere, M.F., Canal, I., Wills, N.M., Atkins, J.F., and Fayet, O. (2011). The interplay of mRNA stimulatory signals required for AUU-mediated initiation and programmed -1 ribosomal frameshifting in decoding of transposable element IS911. Journal of bacteriology 193, 2735-2744.

Ratje, A., Loerke, J., Mikolajka, A., Brünner, M., Hildebrand, P., Starosta, A., Dönhöfer, A., Connell, S., Fucini, P., Mielke, T., et al. (2010). Head swivel on the ribosome facilitates translocation by means of intra-subunit tRNA hybrid sites. Nature 468, 713-716.

Ringquist, S., Shinedling, S., Barrick, D., Green, L., Binkley, J., Stormo, G.D., and Gold, L. (1992). Translation initiation in Escherichia coli: sequences within the ribosome-binding site. Molecular Microbiology 6, 1219-1229.

Ritchie, D.B., Foster, D.A., and Woodside, M.T. (2012). Programmed -1 frameshifting efficiency correlates with RNA pseudoknot conformational plasticity, not resistance to mechanical unfolding. Proc Natl Acad Sci U S A 109, 16167-16172.

Robinson, D.N., and Cooley, L. (1997). Examination of the function of two kelch proteins generated by stop codon suppression. Development 124, 1405-1417.

Rodnina, M., Savelsbergh, A., Katunin, V., and Wintermeyer, W. (1997). Hydrolysis of GTP by elongation factor $\mathrm{G}$ drives tRNA movement on the ribosome. Nature 385, 37-41. 
Rodnina, M.V. (2012). Quality control of mRNA decoding on the bacterial ribosome. Adv Protein Chem Struct Biol 86, 95-128.

Rodnina, M.V., Fricke, R., Kuhn, L., and Wintermeyer, W. (1995). Codon-dependent conformational change of elongation factor Tu preceding GTP hydrolysis on the ribosome. EMBO J 14, 2613-2619.

Rodnina, M.V., Fricke, R., and Wintermeyer, W. (1994). Transient conformational states of aminoacyltRNA during ribosome binding catalyzed by elongation factor Tu. Biochemistry 33, 12267-12275.

Rodnina, M.V., Gromadski, K.B., Kothe, U., and Wieden, H.J. (2005). Recognition and selection of tRNA in translation. FEBS Lett 579, 938-942.

Rodnina, M.V., Savelsbergh, A., Matassova, N.B., Katunin, V.I., Semenkov, Y.P., and Wintermeyer, W. (1999). Thiostrepton inhibits the turnover but not the GTPase of elongation factor $G$ on the ribosome. Proc Natl Acad Sci U S A 96, 9586-9590.

Rodnina, M.V., and Wintermeyer, W. (2001a). Fidelity of aminoacyl-tRNA selection on the ribosome: kinetic and structural mechanisms. Annu Rev Biochem 70, 415-435.

Rodnina, M.V., and Wintermeyer, W. (2001b). Ribosome fidelity: tRNA discrimination, proofreading and induced fit. Trends Biochem Sci 26, 124-130.

Rodnina, M.V., and Wintermeyer, W. (2011). The ribosome as a molecular machine: the mechanism of tRNA-mRNA movement in translocation. Biochem Soc Trans 39, 658-662.

Sanders, C.L., and Curran, J.F. (2007). Genetic analysis of the E site during RF2 programmed frameshifting. Rna 13, 1483-1491.

Savelsbergh, A., Katunin, V.I., Mohr, D., Peske, F., Rodnina, M.V., and Wintermeyer, W. (2003). An elongation factor G-induced ribosome rearrangement precedes tRNA-mRNA translocation. Mol Cell $11,1517-1523$.

Savelsbergh, A., Mohr, D., Kothe, U., Wintermeyer, W., and Rodnina, M. (2005). Control of phosphate release from elongation factor $\mathrm{G}$ by ribosomal protein $\mathrm{L} 7 / 12$. The EMBO journal 24, 4316-4323. 
Savelsbergh, A., Mohr, D., Wilden, B., Wintermeyer, W., and Rodnina, M.V. (2000). Stimulation of the GTPase activity of translation elongation factor $\mathrm{G}$ by ribosomal protein L7/12. J Biol Chem 275, 890894.

Savelsbergh, A., Rodnina, M.V., and Wintermeyer, W. (2009). Distinct functions of elongation factor $\mathrm{G}$ in ribosome recycling and translocation. Rna 15, 772-780.

Schmeing, T., and Ramakrishnan, V. (2009). What recent ribosome structures have revealed about the mechanism of translation. Nature 461, 1234-1242.

Scolnick, E., Tompkins, R., Caskey, T., and Nirenberg, M. (1968). Release factors differing in specificity for terminator codons. Proc Natl Acad Sci U S A 61, 768-774.

Scopes, R.K. (1974). Measurement of protein by spectrophotometry at $205 \mathrm{~nm}$. Analytical biochemistry 59, 277-282.

Sekine, Y., Nagasawa, H., and Ohtsubo, E. (1992). Identification of the site of translational frameshifting required for production of the transposase encoded by insertion sequence IS 1 . Molecular \& general genetics : MGG 235, 317-324.

Selmer, M., Dunham, C., Murphy, F., Weixlbaumer, A., Petry, S., Kelley, A., Weir, J., and Ramakrishnan, V. (2006). Structure of the 70S ribosome complexed with mRNA and tRNA. Science (New York, NY) 313, 1935-1942.

Semenkov, Y., Rodnina, M., and Wintermeyer, W. (1996). The "allosteric three-site model" of elongation cannot be confirmed in a well-defined ribosome system from Escherichia coli. Proceedings of the National Academy of Sciences of the United States of America 93, 12183-12188.

Semenkov, Y., Shapkina, T., Makhno, V., and Kirillov, S. (1992). Puromycin Reaction for the a-SiteBound Peptidyl-Transfer Rna. Febs Letters 296, 207-210.

Semenkov, Y.P., Rodnina, M.V., and Wintermeyer, W. (2000). Energetic contribution of tRNA hybrid state formation to translocation catalysis on the ribosome. Nat Struct Biol 7, 1027-1031. 
Sergiev, P., Lesnyak, D., Kiparisov, S., Burakovsky, D., Leonov, A., Bogdanov, A., Brimacombe, R., and Dontsova, O. (2005). Function of the ribosomal E-site: a mutagenesis study. Nucleic acids research $33,6048-6056$.

Sharma, D., Southworth, D.R., and Green, R. (2004). EF-G-independent reactivity of a pretranslocation-state ribosome complex with the aminoacyl tRNA substrate puromycin supports an intermediate (hybrid) state of tRNA binding. Rna 10, 102-113.

Sharma, P., Xiang, Y., Kato, M., and Warshel, A. (2005). What are the roles of substrate-assisted catalysis and proximity effects in peptide bond formation by the ribosome? Biochemistry 44, 1130711314.

Shehu-Xhilaga, M., Crowe, S., and Mak, J. (2001). Maintenance of the Gag/Gag-Pol ratio is important for human immunodeficiency virus type 1 RNA dimerization and viral infectivity. Journal of virology 75, 1834-1841.

Shine, J., and Dalgarno, L. (1974). 3'-Terminal Sequence of Escherichia-Coli 16s Ribosomal-Rna Possible Role in Initiation and Termination of Protein-Synthesis. P Aust Biochem Soc 7, 72-72.

Sievers, A., Beringer, M., Rodnina, M.V., and Wolfenden, R. (2004). The ribosome as an entropy trap. Proc Natl Acad Sci U S A 101, 7897-7901.

Simonetti, A., Marzi, S., Jenner, L., Myasnikov, A., Romby, P., Yusupova, G., Klaholz, B., and Yusupov, M. (2009). A structural view of translation initiation in bacteria. Cellular and molecular life sciences: CMLS 66, 423-436.

Sipley, J., and Goldman, E. (1993). Increased ribosomal accuracy increases a programmed translational frameshift in Escherichia coli. Proceedings of the National Academy of Sciences of the United States of America 90, 2315-2319.

Somogyi, P., Jenner, A., Brierley, I., and Inglis, S. (1993). Ribosomal pausing during translation of an RNA pseudoknot. Molecular and cellular biology 13, 6931-6940.

Spiegel, P., Ermolenko, D., and Noller, H. (2007). Elongation factor G stabilizes the hybrid-state conformation of the 70S ribosome. RNA (New York, NY) 13, 1473-1482. 
Stahl, G., Ben Salem, S., Li, Z., McCarty, G., Raman, A., Shah, M., and Farabaugh, P.J. (2001). Programmed +1 translational frameshifting in the yeast Saccharomyces cerevisiae results from disruption of translational error correction. Cold Spring Harb Symp Quant Biol 66, 249-258.

Stark, H., Rodnina, M., Wieden, H., van Heel, M., and Wintermeyer, W. (2000). Large-scale movement of elongation factor $\mathrm{G}$ and extensive conformational change of the ribosome during translocation. Cell 100, 301-309.

Steitz, J.A., and Jakes, K. (1975). How ribosomes select initiator regions in mRNA: base pair formation between the $3^{\prime}$ terminus of $16 \mathrm{~S}$ rRNA and the mRNA during initiation of protein synthesis in Escherichia coli. Proc Natl Acad Sci U S A 72, 4734-4738.

Steitz, T. (2008). A structural understanding of the dynamic ribosome machine. Nature reviews Molecular cell biology 9, 242-253.

Steneberg, P., and Samakovlis, C. (2001). A novel stop codon readthrough mechanism produces functional Headcase protein in Drosophila trachea. EMBO reports 2, 593-597.

Sung, D., and Kang, H. (2003). Prokaryotic and eukaryotic translational machineries respond differently to the frameshifting RNA signal from plant or animal virus. Virus Res 92, 165-170.

Takyar, S., Hickerson, R.P., and Noller, H.F. (2005). mRNA helicase activity of the ribosome. Cell 120, 49-58.

Theis, C., Reeder, J., and Giegerich, R. (2008). KnotInFrame: prediction of -1 ribosomal frameshift events. Nucleic Acids Res 36, 6013-6020.

Thiel, V., Ivanov, K.A., Putics, A., Hertzig, T., Schelle, B., Bayer, S., Weissbrich, B., Snijder, E.J., Rabenau, H., Doerr, H.W., et al. (2003). Mechanisms and enzymes involved in SARS coronavirus genome expression. The Journal of general virology 84, 2305-2315.

Tholstrup, J., Oddershede, L.B., and Sorensen, M.A. (2012). mRNA pseudoknot structures can act as ribosomal roadblocks. Nucleic Acids Res 40, 303-313.

Trobro, S., and Aqvist, J. (2005). Mechanism of peptide bond synthesis on the ribosome. Proc Natl Acad Sci U S A 102, 12395-12400. 
Tsuchihashi, Z. (1991). Translational frameshifting in the Escherichia coli dnaX gene in vitro. Nucleic Acids Res 19, 2457-2462.

Tsuchihashi, Z., and Brown, P. (1992). Sequence requirements for efficient translational frameshifting in the Escherichia coli dnaX gene and the role of an unstable interaction between tRNA(Lys) and an AAG lysine codon. Genes \& development 6, 511-519.

Tu, C., Tzeng, T., and Bruenn, J. (1992). Ribosomal movement impeded at a pseudoknot required for frameshifting. Proceedings of the National Academy of Sciences of the United States of America 89, 8636-8640.

Tujebajeva, R.M., Copeland, P.R., Xu, X.M., Carlson, B.A., Harney, J.W., Driscoll, D.M., Hatfield, D.L., and Berry, M.J. (2000). Decoding apparatus for eukaryotic selenocysteine insertion. EMBO reports 1, 158-163.

Uemura, S., Aitken, C.E., Korlach, J., Flusberg, B.A., Turner, S.W., and Puglisi, J.D. (2010). Real-time tRNA transit on single translating ribosomes at codon resolution. Nature 464, 1012-1017.

Urbonavicius, J., Qian, Q., Durand, J., Hagervall, T., and Björk, G. (2001). Improvement of reading frame maintenance is a common function for several tRNA modifications. The EMBO journal 20, 4863-4873.

Valle, M., Zavialov, A., Sengupta, J., Rawat, U., Ehrenberg, M., and Frank, J. (2003). Locking and unlocking of ribosomal motions. Cell 114, 123-134.

Weiner, A., and Weber, K. (1971). Natural read-through at the UGA termination signal of Q-beta coat protein cistron. Nature: New biology 234, 206-209.

Weiss, R., Dunn, D., Atkins, J., and Gesteland, R. (1990). Ribosomal frameshifting from -2 to +50 nucleotides. Progress in nucleic acid research and molecular biology 39, 159-183.

Weiss, R.B., Dunn, D.M., Shuh, M., Atkins, J.F., and Gesteland, R.F. (1989). E. coli ribosomes re-phase on retroviral frameshift signals at rates ranging from 2 to 50 percent. New Biol 1, 159-169. 
Wen, J.D., Lancaster, L., Hodges, C., Zeri, A.C., Yoshimura, S.H., Noller, H.F., Bustamante, C., and Tinoco, I. (2008). Following translation by single ribosomes one codon at a time. Nature 452, 598603.

Wilden, B., Savelsbergh, A., Rodnina, M.V., and Wintermeyer, W. (2006). Role and timing of GTP binding and hydrolysis during EF-G-dependent tRNA translocation on the ribosome. Proc Natl Acad Sci U S A 103, 13670-13675.

Wills, N.M. (2010). Translational Bypassing-Peptidyl-tRNA Re-pairing at Non-overlapping Sites. Springer, 365-381.

Wills, N.M., Gesteland, R.F., and Atkins, J.F. (1991). Evidence that a downstream pseudoknot is required for translational read-through of the Moloney murine leukemia virus gag stop codon. Proc Natl Acad Sci U S A 88, 6991-6995.

Wills, N.M., Moore, B., Hammer, A., Gesteland, R.F., and Atkins, J.F. (2006). A functional -1 ribosomal frameshift signal in the human paraneoplastic Ma3 gene. J Biol Chem 281, 7082-7088.

Wilson, W., Malim, M.H., Mellor, J., Kingsman, A.J., and Kingsman, S.M. (1986). Expression strategies of the yeast retrotransposon Ty: a short sequence directs ribosomal frameshifting. Nucleic Acids Res $14,7001-7016$.

Wintermeyer, W., and Zachau, H.G. (1979). Fluorescent derivatives of yeast tRNAPhe. European journal of biochemistry / FEBS 98, 465-475.

Wohlgemuth, I., Brenner, S., Beringer, M., and Rodnina, M.V. (2008). Modulation of the rate of peptidyl transfer on the ribosome by the nature of substrates. J Biol Chem 283, 32229-32235.

Yarian, C., Marszalek, M., Sochacka, E., Malkiewicz, A., Guenther, R., Miskiewicz, A., and Agris, P. (2000). Modified nucleoside dependent Watson-Crick and wobble codon binding by tRNALysUUU species. Biochemistry 39, 13390-13395.

Yelverton, E., Lindsley, D., Yamauchi, P., and Gallant, J. (1994). The function of a ribosomal frameshifting signal from human immunodeficiency virus-1 in Escherichia coli. Molecular microbiology 11, 303-313. 
Yokoyama, S., and Nishimura, S. (1995). tRNA: structure, biosynthesis, and function. Washington, DC: American Society for Microbiology, 207-223.

Youngman, E., McDonald, M., and Green, R. (2008). Peptide release on the ribosome: mechanism and implications for translational control. Annual review of microbiology 62, 353-373.

Yu, C.H., Noteborn, M.H., and Olsthoorn, R.C. (2010). Stimulation of ribosomal frameshifting by antisense LNA. Nucleic Acids Res 38, 8277-8283.

Yusupov, M.M., Yusupova, G.Z., Baucom, A., Lieberman, K., Earnest, T.N., Cate, J.H., and Noller, H.F. (2001). Crystal structure of the ribosome at 5.5 A resolution. Science $292,883-896$.

Yusupova, G., Jenner, L., Rees, B., Moras, D., and Yusupov, M. (2006). Structural basis for messenger RNA movement on the ribosome. Nature 444, 391-394.

Yusupova, G., Yusupov, M., Cate, J., and Noller, H. (2001). The path of messenger RNA through the ribosome. Cell 106, 233-241.

Zaher, H.S., and Green, R. (2009). Quality control by the ribosome following peptide bond formation. Nature 457, 161-166. 


\section{ACKNOWLEDGEMENTS}

I would like to thank Marina Rodnina, for accepting me in her lab and offering me to work in a project, which I find truly fascinating. Being in this lab has been a great experience for me. I also thank Wolfgang Wintermeyer for his advices and ideas.

I would like to thank especially to Frank Peske, for all his time and help in my PhD work. I learned a lot from his bright ideas and our stimulating discussions.

Thanks to Vladimir Katunin, who is unfortunately not with us anymore, for being a great collaborator and for sharing his experiences with me. He started the work on frameshifting and graciously supplied me with the tRNAs and many useful methods.

I would like to thank my students Deniz Ugurlu and Merve Öztürk, whom I trained during their summer internships for their critical questions and enthusiasm, which also trained me as a scientist.

This work would be very complicated without the reagents and common stocks prepared by the technical facility in our lab, therefore I would like to thank all, and thanks to Dimitra for simplifying the official work. Especially, I would like to thank Michael Zimmermann for being my second hands during my pregnancy, Olaf Geintzer for the fastest tRNA preparations and Sandra Kappler for the ribosome and EF-Tu that were prepared only for this project. I would also like to thank Anja Lehweß-Litzmann, Carlos da Cunha and Riccardo Belardinelli, for sharing their valuable preps with me and for helpful discussions.

I would like to express my gratitude to the IMPRS Molecular Biology Program, being a part of this program is one of the highlights in my life and my thesis committee members for their availability and helpful ideas. Especially, I would like to thank our program coordinator Steffen Burkhardt for his help and advices.

I would like to thank all my friends for all the fun and conversations, but especially for being there at frustrating times. Thanks to my great helper mother and especially to my father who could not see the completion of my work, for their encouragement and confidence in me. 
This work is dedicated to my life partner Kemal, who always believed in me, supported and pushed me to succeed. He is the one encouraging me and gives me the strength that keeps me going. Last words go to my little sunshine Alya, who joined us 8 months before the completion of this work. I would like to thank her to keep herself busy and happy while I was preparing my dissertation. She is the light in my life; I am learning something new about life from her every day. 


\section{APPENDIX A: In vivo dual luciferase assay of -1 frameshifting in dnaX}

\section{A.1 Construction of the plasmids}

The backbone vector (described in section 4.7.1) was utilized for the construction of the plasmids used in the dnaX in vivo dual luciferase assay. The dnaX fragment was cloned from the $E$. coli BL21 genome. The dnaX fragment was blunt end ligated to the Fluc-Rluc parental vector. This vector served as a template for site directed mutagenesis to generate all the constructs for E. coli dnaX frameshifting tests. Site directed mutagenesis was performed as described in section 4.7.2 using the primers given in Table 8. Mutations were verified by sequencing (Seqlab, Göttingen). The In vivo luciferase assay was performed as described in section 4.8 .

Table 8. List of primers used to construct the dnaX in vivo luciferase assay.

\begin{tabular}{cl}
\hline Vector & Primer Pair \\
\hline 1 & 5' AGAACGCTGCGCTGGAAAGACT 3' \\
& 5' TTAACTCTTCTTCGCTTTGGTTGCTC 3' \\
2 & 5' GAAAGTCCAAATTGCAGCAGGCACCGACT 3' \\
& 5' AGTCGGTGCCTGCTGCAATTTGGACTTTC 3' \\
3 & 5' AGGGAGCAACCAAAGCGAAGAAGAGTTAACCGGCAGCCGCTAC 3' \\
& 5' GTAGCGGCTGCCGGTTAACTCTTCTTCGCTTTGGTTGCTCCCT 3' \\
4 & 5' AGAACGCTGCGCTGGAAAGACT 3' \\
& 5' TTAACTCTTTTTTGCTTTGGTTGCTCC 3' \\
5 & 5' AGAACGCTGCGCTGGAAAGACT 3' \\
& 5' TTAACTCTTCTTCGCTTTGGTTGCTC 3' \\
6 & 5' AACCAAAGCAAAAAAGAGTTAACCGGCAGCCGCTA 3' \\
& 5' TAGCGGCTGCCGGTTAACTCTTTTTTGCTTTGGTT 3'
\end{tabular}




\section{A.2 Results}

The dnaX gene is the one of the two cellular genes found to be expressed by programmed -1 ribosomal frameshifting (Blinkowa and Walker, 1990; Mejlhede et al., 1999). Two subunits gamma, $\gamma$ and tau, $\tau$ of the DNA polymerase III holoenzyme are synthesized from the overlapping ORFs present in the $d n a X$ gene. For efficient replication, the correct ratio of the synthesized subunits is of critical importance (Blinkowa and Walker, 1990; Flower and McHenry, 1990; Tsuchihashi, 1991). This is an elegant example of how -1 frameshifting influences DNA replication.

In this study, using the dnax gene as a model we set up an in vivo dual luciferase assay system in order to study recoding signals in bacteria. The slippery sequence of dnaX (A AAA $A A G)$ leads to efficient frameshifting in bacteria. In the frameshifting site the lysine codon is encoded at both codons of the slippery site. A downstream stem loop is present 6 nucleotides downstream of the slippery A AAA AAG stretch, which has a length of 29 nucleotides. The GC rich stem loop (predicted free energy of $14.5 \mathrm{kcal} / \mathrm{mol}$ ) is composed of a long stem with a single mismatch and a shorter loop of 5 nucleotides (Figure 20) (Larsen et al., 1997).

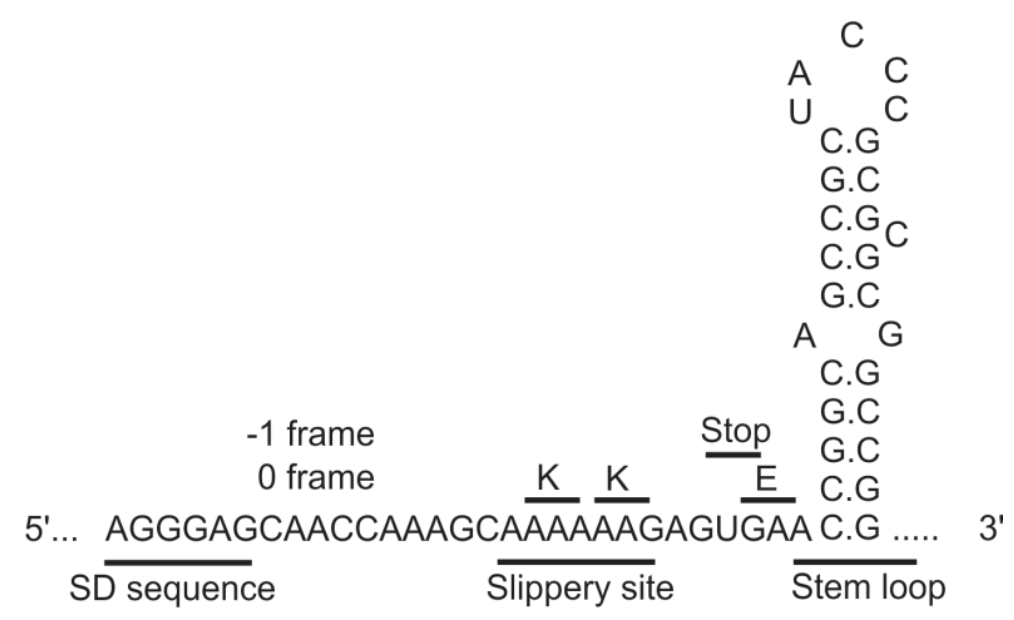

Figure 20. The dnaX frameshifting cassette. The underlined A AAA AAG is the slippery site and the stop codon is in the -1 reading frame.

To study -1 frameshifting in vivo, the dual luciferase construct was designed such that translation of renilla luciferase was a measure of efficiency of read-through translation or -1 frameshifting of the inserted dnaX gene fragment. Full length firefly-renilla phusion protein 
was expressed only if the ribosomes would shift to the -1 frame while translating the dnaX gene fragment. Firefly luciferase was used as an internal reference gene to compare efficiency of in frame translation from each expression vector relative to each other (Figure 21A).

Table 9. Vectors used for the dnaX in vivo luciferase assay. Nucleotides forming the slippery site are underlined.

\begin{tabular}{|c|c|c|c|}
\hline Vector & Recoding site & Stem loop & Rluc frame \\
\hline 1 & $5^{\prime} \ldots$ AAAGCGAAGAAGAGTTA & & 0 \\
\hline 2 & $5^{\prime} \ldots$ AAAGCAAAAAAGAGTGA & ACCGGCAGCCGCTACCCGCGCGCGGCCGGT...3’ & 0 \\
\hline 3 & $5^{\prime} \ldots$ AAAGCGAAGAAGAGTTA & & -1 \\
\hline 4 & $5^{\prime} \ldots$ AAAGCAAAAAAGAGTT & & -1 \\
\hline 5 & $5^{\prime} \ldots$ AAAGCGAAGAAGAGTTA & ACCGGCAGCCGCTACCCGCGCGCGGCCGGT...3’ & -1 \\
\hline 6 & $5^{\prime} \ldots$ AAAGCAAAAAAGAGTTA & & -1 \\
\hline
\end{tabular}

The efficiency of translation in all vectors used in this study (given in Table 9) was normalized to control construct without frameshifting elements (\#1), defined as $100 \%$. In the frameshifting construct (\#6), 50\% frameshifting was reported, indicating half of the ribosomes shift the reading frame during translation of the dnaX linker region. The in-frame control for frameshifting (\#2) was translated by $44 \%$ efficiency. Hereafter, we examined the effect of single frameshifting stimulatory element during translation of the dnaX gene. Deletion of the downstream stem loop region (\#4) decreased the efficiency of frameshifting to $25 \%$, indicating that slippery sequence is sufficient to induce frameshifting in E. coli. Interestingly, in the absence of the slippery site $(\# 3)$, we reported $10 \%$ translation in the -1 reading frame. This suggests that other stimulatory elements such as the stem loop can stimulate frameshifting up to some extent. We have generated another mutant in which slippery site and stem loop were removed (\#5). In this case still low level of translation was observed from the -1 reading frame, which can be explained by either the leakiness of the translation system or influence of other potential frameshifting stimulators, such as the SD sequence. One can also speculate the upstream AAAG which is located 1 nucleotide away 
from the slippery site might cause tRNA slippage, thereby the ribosomes might shift the reading frame (Figure 21B).

A

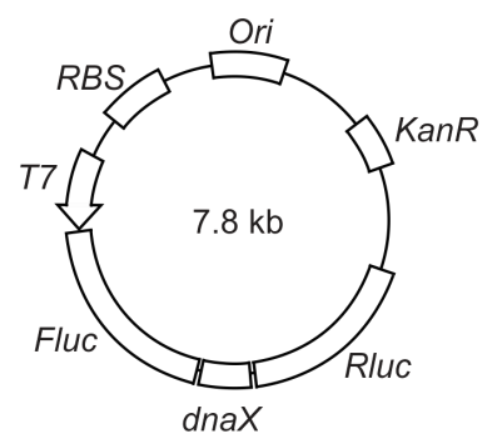

B

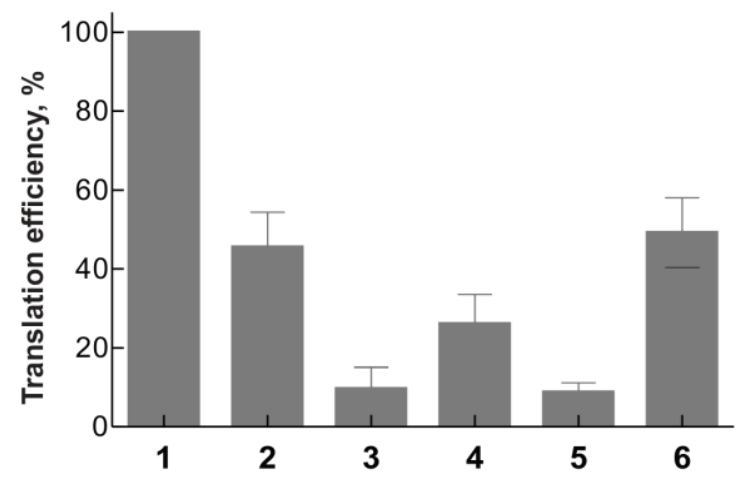

Figure 21. In vivo dual luciferase assay in dnaX gene.

(A) A dual luciferase vector is constructed in which expression of dnaX linker in different reading frames can be reported from the expression of the phusion protein Fluc-Rluc.

(B) Translation efficiencies of various constructs (1-6) created to investigate effect of different stimulatory elements on in vivo frameshifting in $E$. coli dnaX gene. (1) control vector (-//0), (2) frameshifting, Rluc zero frame vector $(+/+/ 0)$, (3) stem loop alone, Rluc -1 frame vector $(-/+/-1)$, (4) slippery site alone, Rluc -1 frame vector $(+/-/-1)$, (5) control vector, Rluc -1 frame $(-/-/-1),(6)$ frameshifting vector $(+/+/-1)$.

\section{A.3 Discussion}

Here we present the result of the in vivo dual luciferase assay of dnaX, which was developed to study recoding signals in $E$. coli.

There are several advantages of the in vivo dual luciferase assay system over the previously used reporter systems. The first advantage is that the use of the bicystronic reporter enables the internal control of the gene expression, such that normalization by optical density is not required. In addition, measurement of total protein concentration is not required since the relative activity measurement makes it possible to compare protein expression among different samples. The next advantage was control of gene expression through a single vector. Expression from the single vector was under the control of T7 promoter, which assured that mRNA abundance does not result in differences between the two reporter genes. In addition, the sensitivity of the luciferase assay system is much higher compared to other protein expression tests such as the lac Z ( $\beta$-galactosidase) based assays. 
The applicability of the dual luciferase assay system to study recoding signals was tested in various systems. Up to now, our lab successfully employed the in vivo dual reporter assay for studying frameshifting of the bacterial dnaX gene, viral IBV $1 a / 1 b$ gene and for selenocysteine incorporation (by S. B. Kotini). To develop the in vivo reporter assay the dnaX gene of $E$. coli was a suitable candidate due to the high frameshifting efficiency $(50-80 \%)$ observed in vivo (Larsen et al., 1997; Tsuchihashi and Brown, 1992). In our in vivo assay system we have observed $50 \%$ efficiency of frameshifting for the dnaX gene, indicating that half of the ribosomes shift the reading frame during translation, consistent with the required ratio 1:1 to obtain gamma and tau subunits of DNA polymerase III (Flower and McHenry, 1990). We have studied individual elements of -1 frameshifting in E. coli by introducing various mutations that disrupt the slippery sequence element and/or the stem loop. We observed efficient translation from the -1 reading frame even in the absence of a slippery site. This shows that the stem loop can be a sufficient stimulator for -1 ribosomal frameshifting. Similarly, removal of the stem loop did not cause abolishment of frameshifting. Our results supported that both a slippery site and a stem loop are required for efficient frameshifting, yet frameshifting can still take place to a certain extent in the presence of a single stimulatory element. We emphasize that there is the potential effect of the SD region 10 nucleotides upstream of the slippery site, which was not checked in our system, but has been linked with frameshifting previously (Larsen et al., 1997). These imply an additive effect of various stimulatory elements on frameshifting, which act in a concerted manner. These support the results that were obtained from the IBV $1 a / 1 b$ frameshifting and demonstrate a difference in the way the eukaryotic and prokaryotic ribosomes translate the frameshifting sites. 
APPENDIX B: List of abbreviations

\begin{tabular}{|c|c|}
\hline$\AA$ & Angstrom \\
\hline${ }^{\circ} \mathrm{C}$ & degree Celsius \\
\hline aа & amino acid \\
\hline Amp & ampicillin \\
\hline aSD & anti-Shine-Dalgarno \\
\hline$B W Y(V)$ & beet western yellows (virus) \\
\hline BYDV & barley yellow dwarf virus \\
\hline DTT & dithiotheretiol \\
\hline E. coli & Escherichia coli \\
\hline eEF-2 & Eukaryotic elongation factor-2 \\
\hline EF-G & Elongation factor-G \\
\hline EF-Tu & Elongation factor-Tu \\
\hline $\mathrm{F}$ & phenylalanine \\
\hline Fluc & firefly luciferase \\
\hline $\mathrm{fM}(\mathrm{M})$ & formyl-methionine (methionine) \\
\hline fMet & formyl-methionine \\
\hline GTP & guanosine triphosphate \\
\hline $\mathrm{h}$ & hour \\
\hline IBV & Infectious bronchitis virus \\
\hline IC & Initiation complex \\
\hline $\mathrm{K}$ & lysine \\
\hline Kan & kanamycin \\
\hline $\mathrm{L}$ & leucine \\
\hline LB-medium & Luria-Bertani-medium \\
\hline
\end{tabular}




\begin{tabular}{|c|c|}
\hline Leu & leucine \\
\hline Lys & lysine \\
\hline M & molar \\
\hline $\min$ & minute \\
\hline MDa & megadalton \\
\hline MMTV & mouse mammary tumor virus \\
\hline $\mathrm{nt}$ & nucleotide \\
\hline OD & optical density \\
\hline Phe & phenylalanine \\
\hline Pmn & puromycin \\
\hline Rluc & renilla luciferase \\
\hline RP-HPLC & reversed phase-high pressure liquid chromatography \\
\hline $\mathrm{RS}(\mathrm{V})$ & Raus sarcoma virus \\
\hline RT & room temperature \\
\hline S & Svedberg unit \\
\hline s & second \\
\hline SD & Shine-Dalgarno \\
\hline Tyr & tyrosine \\
\hline $\mathrm{V}$ & valine \\
\hline$v / v$ & volume per volume \\
\hline Val & valine \\
\hline VMV & Visna-Maedi virus \\
\hline Y & tyrosine \\
\hline
\end{tabular}




\section{APPENDIX C: CURRICULUM VITAE}

\section{Personal Information}

Name : Neva Caliskan

Date of Birth $\quad$ : $\quad 12.08 .1983$

Place of Birth : Ankara

\section{Education}

06/2009 - present

Ph.D. studies at the Department of Physical Biochemistry

Max Planck Institute for Biophysical Chemistry, Göttingen

$11 / 2008-04 / 2009$

M.Sc. studies at the Department of Molecular Cell Biology

Max Planck Institute for Biophysical Chemistry, Göttingen

09/2007 - present

International Max Planck Research School

Georg August University Göttingen

09/2001-05/2006

B.Sc. in Molecular Biology and Genetics

Middle East Technical University, Ankara, Turkey 